
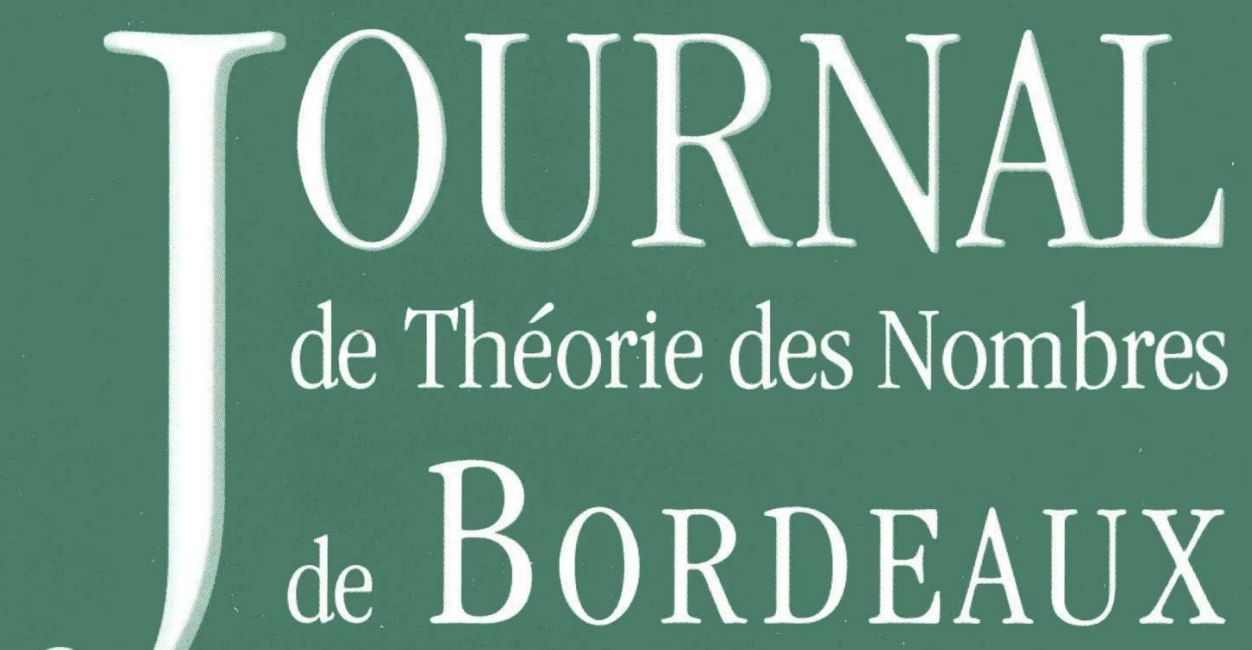

anciennement Séminaire de Théorie des Nombres de Bordeaux

\title{
Gaëtan CHENEVIER
}

The characteristic masses of Niemeier lattices

Tome 32, no 2 (2020), p. 545-583.

$<$ http://jtnb.centre-mersenne.org/item?id=JTNB_2020__32_2_545_0>

(C) Société Arithmétique de Bordeaux, 2020, tous droits réservés. L'accès aux articles de la revue «Journal de Théorie des Nombres de Bordeaux » (http://jtnb.centre-mersenne.org/), implique l'accord avec les conditions générales d'utilisation (http://jtnb. centre-mersenne.org/legal/). Toute reproduction en tout ou partie de cet article sous quelque forme que ce soit pour tout usage autre que l'utilisation à fin strictement personnelle du copiste est constitutive d'une infraction pénale. Toute copie ou impression de ce fichier doit contenir la présente mention de copyright.

\section{cedram}

Article mis en ligne dans le cadre du

Centre de diffusion des revues académiques de mathématiques

http://www.centre-mersenne.org/ 


\title{
The characteristic masses of Niemeier lattices
}

\author{
par GAËTAN CHENEVIER
}

RÉSUMÉ. Soient $L$ un réseau entier d'un espace euclidien $E$ de dimension $n$ et $W$ une représentation irréductible du groupe orthogonal de $E$. Nous donnons un algorithme calculant la dimension du sous-espace des éléments de $W$ invariants par le groupe $\mathrm{O}(L)$ des isométries de $L$. Une étape clef est de déterminer, pour tout polynôme $P$, la proportion des éléments de $\mathrm{O}(L)$ de polynôme caractéristique $P$, une collection de nombres rationnels que nous appelons les masses caractéristiques de $L$. En guise d'application, nous déterminons les masses caractéristiques de tous les réseaux de Niemeier, et plus généralement de tous les réseaux pairs de déterminant $\leq 2$ en dimension $n \leq 25$.

Pour les réseaux de Niemeier, en guise de vérification, nous donnons une méthode alternative (et humaine) pour calculer leurs masses caractéristiques. L'ingrédient principal est la détermination, pour chaque réseau de Niemeier $L$ de système de racines $R$ non vide, des $\mathrm{G}(R)$-classes de conjugaison d'éléments du sous-groupe «ombral » $\mathrm{O}(L) / \mathrm{W}(R)$ de $\mathrm{G}(R)$, où $\mathrm{G}(R)$ est le groupe des automorphismes du diagramme de Dynkin de $R$, et $\mathrm{W}(R)$ son groupe de Weyl.

Ces résultats ont des applications à l'étude des espaces de formes automorphes des groupes orthogonaux de formes quadratiques sur $\mathbb{Q}$ définies positives: nous donnons des formules concrètes pour la dimension de ces espaces en niveau 1 , comme fonction du poids $W$, en tout rang $n \leq 25$.

AbSTRACT. Let $L$ be an integral lattice in an $n$-dimensional Euclidean space $E$ and $W$ an irreducible representation of the orthogonal group of $E$. We give an implemented algorithm computing the dimension of the subspace of invariants in $W$ under the isometry group $\mathrm{O}(L)$ of $L$. A key step is the determination, for any polynomial $P$, of the proportion of elements in $\mathrm{O}(L)$ with characteristic polynomial $P$, a collection of rational numbers that we call the characteristic masses of $L$. As an application, we determine the characteristic masses of all the Niemeier lattices, and more generally of any even lattice of determinant $\leq 2$ in dimension $n \leq 25$.

For Niemeier lattices, as a verification, we provide an alternative (human) computation of the characteristic masses. The main ingredient is the determination, for each Niemeier lattice $L$ with non-empty root system $R$, of the $\mathrm{G}(R)$-conjugacy classes of the elements of the "umbral" subgroup $\mathrm{O}(L) / \mathrm{W}(R)$

Manuscrit reçu le 13 février 2020, révisé le 24 mars 2020, accepté le 25 avril 2020.

2020 Mathematics Subject Classification. 11F, 11F55, 11H55, 11H56, 11H71, 20D08, $22 \mathrm{C} 05$.

Mots-clefs. Euclidean lattices, isometry groups, Niemeier lattices, automorphic forms.

During this work, the author has been supported by the C.N.R.S. and by the projects ANR14-CE25-0002-01 PERCOLATOR and ANR-19-CE40-0015-02 COLOSS. 
of $\mathrm{G}(R)$, where $\mathrm{G}(R)$ is the automorphism group of the Dynkin diagram of $R$, and $\mathrm{W}(R)$ its Weyl group.

These results have consequences for the study of the spaces of automorphic forms of the definite orthogonal groups in $n$ variables over $\mathbb{Q}$. As an example, we provide concrete dimension formulas in the level 1 case, as a function of the weight $W$, up to $n=25$.

\section{Introduction}

1.1. A motivation: dimension of spaces of level 1 automorphic forms for $\mathbf{O}_{n}$. Let $n$ be an integer $\equiv 0 \bmod 8$ and let $\mathcal{L}_{n}$ be the set of all even unimodular lattices in the standard Euclidean space $\mathbb{R}^{n}$. A standard example of an element of $\mathcal{L}_{n}$ is the lattice $\mathrm{E}_{n}=\mathrm{D}_{n}+\mathbb{Z} \frac{1}{2}(1,1, \ldots, 1)$, where $\mathrm{D}_{n}$ denotes the subgroup of elements $\left(x_{i}\right)$ in $\mathbb{Z}^{n}$ with $\sum_{i} x_{i} \equiv 0 \bmod 2$. The orthogonal group $\mathrm{O}\left(\mathbb{R}^{n}\right)$ naturally acts on $\mathcal{L}_{n}$, with finitely many orbits, and we set

$$
\mathrm{X}_{n} \stackrel{\text { det }}{=} \mathrm{O}\left(\mathbb{R}^{n}\right) \backslash \mathcal{L}_{n} .
$$

Representatives of this set $\mathrm{X}_{n}$ have been determined so far for $n \leq 24$ only: we have $\mathrm{X}_{8}=\left\{\mathrm{E}_{8}\right\}$ (Mordell), $\mathrm{X}_{16}=\left\{\mathrm{E}_{8} \oplus \mathrm{E}_{8}, \mathrm{E}_{16}\right\}$ (Witt) and $\left|\mathrm{X}_{24}\right|=24$ (Niemeier): see $[18,32,38,44]$. The elements of $\mathcal{L}_{24}$, to which we shall refer as the Niemeier lattices, will play a major role in this paper. Similarly, for $n \equiv \pm 1 \bmod 8$ we define $\mathcal{L}_{n}$ as the set of all even lattices with covolume $\sqrt{2}$ in $\mathbb{R}^{n}$, as well as $\mathrm{X}_{n}$ by the same Formula (1.1). In this case, representatives of $\mathrm{X}_{n}$ are known up to $n=25$, this last (and most complicated) case being due to Borcherds [3], and we have

$$
\left|\mathrm{X}_{1}\right|=\left|\mathrm{X}_{7}\right|=\left|\mathrm{X}_{9}\right|=1,\left|\mathrm{X}_{15}\right|=2,\left|\mathrm{X}_{17}\right|=4,\left|\mathrm{X}_{23}\right|=32 \text { and }\left|\mathrm{X}_{25}\right|=121 .
$$

For any $n \equiv-1,0,1 \bmod 8$, and any complex, finite dimensional, continuous, linear representation $W$ of $\mathrm{O}\left(\mathbb{R}^{n}\right)$, we consider the complex vector space of $W$-valued $\mathrm{O}\left(\mathbb{R}^{n}\right)$-equivariant functions on $\mathcal{L}_{n}$ :

$$
\mathrm{M}_{W}\left(\mathrm{O}_{n}\right)=\left\{f: \mathcal{L}_{n} \rightarrow W \mid f(g L)=g f(L) \forall L \in \mathcal{L}_{n}, \forall g \in \mathrm{O}\left(\mathbb{R}^{n}\right)\right\}
$$

This space has a natural interpretation as a space of level 1 and weight $W$ automorphic forms for the orthogonal group scheme $\mathrm{O}_{n}$ of any element of $\mathcal{L}_{n}$. In particular, it has a very interesting action of the Hecke ring of $\mathrm{O}_{n}$ (see e.g. [8, §4]), which is a first indication of our interest in it.

If $L$ is a lattice in the Euclidean space $\mathbb{R}^{n}$, we denote by $\mathrm{O}(L)=\{g \in$ $\left.\mathrm{O}\left(\mathbb{R}^{n}\right) \mid g L=L\right\}$ its (finite) isometry group. If $W$ is a representation of $\mathrm{O}\left(\mathbb{R}^{n}\right)$, we denote by $W^{\Gamma}=\{w \in W \mid \gamma w=w, \forall \gamma \in \Gamma\} \subset W$ the subspace of invariants of the subgroup $\Gamma$ of $\mathrm{O}\left(\mathbb{R}^{n}\right)$. Fix representatives 
$L_{1}, \ldots, L_{h}$ of the classes in $\mathrm{X}_{n}$. Then the map $f \mapsto\left(f\left(L_{i}\right)\right)$ induces a $\mathbb{C}$ linear isomorphism

$$
\mathrm{M}_{W}\left(\mathrm{O}_{n}\right) \stackrel{\sim}{\rightarrow} \prod_{i=1}^{h} W^{\mathrm{O}\left(L_{i}\right)} .
$$

It follows that $\mathrm{M}_{W}\left(\mathrm{O}_{n}\right)$ is finite dimensional. Our main aim in this work, which is of computational flavor, is to explain how to compute $\operatorname{dim} \mathrm{M}_{W}\left(\mathrm{O}_{n}\right)$ for all $n \leq 25$ and $W$ arbitrary. The special cases $n=7,8,9$ and $n=16$, more precisely their SO-variants ${ }^{1}$, had been respectively previously considered in [9, Ch. 2] and in [8, Ch. IX Prop. 5.13]. In a different direction, see Appendix B for an asymptotic formula for $\operatorname{dim} \mathrm{M}_{W}\left(\mathrm{O}_{n}\right)$ (for any $n$ ).

Our main motivation for these computations is the relation between the spaces $\mathrm{M}_{W}\left(\mathrm{O}_{n}\right)$ and geometric $\ell$-adic representations of $\operatorname{Gal}(\overline{\mathbb{Q}} / \mathbb{Q})$ of Artin conductor 1 (or pure motives over $\mathbb{Q}$ with good reduction everywhere) which follows from the general yoga and point of views of Langlands and Arthur on automorphic representations. This circle of ideas has been studied in great details in the recent works [8, 9], and pursued in $[11,43]$, to which we refer to for further explanations. As a start, the reader may consult the preface of [8]. Let us simply say here that in a forthcoming work of Taibi and the author, we shall use the results of the present paper as an ingredient to extend to higher dimensions $d$, hopefully up to $d=24$, the counting of level 1, algebraic, essentially selfdual cuspidal automorphic representations of $\mathrm{GL}_{d}$ over $\mathbb{Q}$ started in the aforementioned works. One alternative motivating goal of these works is to obtain new information on the size of $\mathrm{X}_{31}$ and $\mathrm{X}_{32}$ (see e.g. [8, Thm. IX.6.1] for a direct proof of the equality $\left|\mathrm{X}_{24}\right|=24$ not relying on any lattice computation).

1.2. Dimension of invariants and characteristic masses. Consider now an arbitrary integral lattice $L$ in the standard Euclidean space $\mathbb{R}^{n}$ of arbitrary dimension $n$, and a finite dimensional representation $W$ of $\mathrm{O}\left(\mathbb{R}^{n}\right)$. Motivated by the previous paragraph, we are interested in algorithms to determine the dimension of the subspace $W^{\mathrm{O}(L)} \subset W$ of $\mathrm{O}(L)$-invariants in $W$. Of course, our requirement will be that these algorithms be efficient for the even lattices of determinant $\leq 2$, as in Section 1.1.

Obviously, we may and do assume that $W$ is irreducible. It will be convenient to parameterize the isomorphism classes of irreducible complex representations of $\mathrm{O}\left(\mathbb{R}^{n}\right)$, following Weyl's original approach [46], by the $n$ permissible $^{2}$ (integer) partitions: see Appendix A for a brief reminder of

\footnotetext{
${ }^{1}$ We define $\mathrm{M}_{W}\left(\mathrm{SO}_{n}\right)$ by replacing $\mathrm{O}\left(\mathbb{R}^{n}\right)$ with $\mathrm{SO}\left(\mathbb{R}^{n}\right)$ in $(1.2)$, and $W$ with a representation of $\mathrm{SO}\left(\mathbb{R}^{n}\right)$. We have then $\mathrm{M}_{W}\left(\mathrm{SO}_{n}\right) \simeq \mathrm{M}_{W^{\prime}}\left(\mathrm{O}_{n}\right)$ where $W^{\prime}$ is the representation of $\mathrm{O}\left(\mathbb{R}^{n}\right)$ induced from $W[8, \S 4.4 .4]$. The question of computing dimensions in the SO-case is thus a special case of the same question in the O-case (the one considered here).

2 This means that the first two columns of the Young diagram of the partition have at most $n$ boxes in total.
} 
this parameterization and its relation with the highest weight theory for $\mathrm{SO}\left(\mathbb{R}^{n}\right)$. This parameterization not only allows to deal with the two connected components of $\mathrm{O}\left(\mathbb{R}^{n}\right)$ in a very concise way, but it is also especially relevant for the character formulas we shall use.

We denote by $\mathrm{W}_{\lambda}$ an irreducible representation of $\mathrm{O}\left(\mathbb{R}^{n}\right)$ associated with the $n$-permissible partition $\lambda=\left(\lambda_{1} \geq \cdots \geq \lambda_{n} \geq 0\right)$. The element $-\mathrm{id}_{n}$ acts on $\mathrm{W}_{\lambda}$ by the $\operatorname{sign}(-1)^{|\lambda|}$, with $|\lambda|=\sum_{i} \lambda_{i}$, so $\mathrm{W}_{\lambda}^{\mathrm{O}(L)}$ vanishes for $|\lambda| \equiv 1 \bmod 2$. Our starting point is the trivial formula $\operatorname{dim} \mathrm{W}_{\lambda}^{\mathrm{O}(L)}=$ $\frac{1}{|\mathrm{O}(L)|} \sum_{\gamma \in \mathrm{O}(L)} \operatorname{Trace}\left(\gamma ; \mathrm{W}_{\lambda}\right)$, that we rewrite as

$$
\operatorname{dim} \mathrm{W}_{\lambda}^{\mathrm{O}(L)}=\sum_{P \in \mathrm{Car}_{n}} \mathrm{~m}_{\mathrm{O}(L)}(P) \operatorname{Trace}\left(\mathrm{c}_{P} ; \mathrm{W}_{\lambda}\right)
$$

where:

(i) $\operatorname{Car}_{n} \subset \mathbb{Z}[t]$ denotes the (finite) subset of polynomials of degree $n$ which are products of cyclotomic polynomials. This subset is ${ }^{3}$ also the set of characteristic polynomials of the elements of $\mathrm{O}\left(\mathbb{R}^{n}\right)$ preserving some lattice in $\mathbb{R}^{n}$. Using the irreducibility of cyclotomic polynomials in $\mathbb{Q}[t]$, it is straightforward to enumerate the elements of $\mathrm{Car}_{n}$ for small $n$ with the help of a computer: see Table 1.1 for the cardinality of $\mathrm{Car}_{n}$ for $n \leq 27$ (sequence A120963 on the OEIS [42]).

TABLE 1.1. The cardinality of $\operatorname{Car}_{n}$ for $n \leq 27$.

\begin{tabular}{c|c|c|c|c|c|c|c|c|c}
$n$ & 1 & 2 & 3 & 4 & 5 & 6 & 7 & 8 & 9 \\
\hline$\left|\operatorname{Car}_{n}\right|$ & 2 & 6 & 10 & 24 & 38 & 78 & 118 & 224 & 330 \\
\hline$n$ & 10 & 11 & 12 & 13 & 14 & 15 & 16 & 17 & 18 \\
\hline$\left|\operatorname{Car}_{n}\right|$ & 584 & 838 & 1420 & 2002 & 3258 & 4514 & 7134 & 9754 & 15010 \\
\hline$n$ & 19 & 20 & 21 & 22 & 23 & 24 & 25 & 26 & 27 \\
\hline$\left|\operatorname{Car}_{n}\right|$ & 20266 & 30532 & 40798 & 60280 & 79762 & 115966 & 152170 & 217962 & 283754
\end{tabular}

(ii) For any finite subset $S \subset \mathrm{O}\left(\mathbb{R}^{n}\right)$, and any $P$ in $\mathbb{R}[t]$, we denote by $\mathrm{m}_{S}(P)$ the number of elements $g$ in $S$ with $\operatorname{det}\left(t \mathrm{id}_{n}-g\right)=P$, divided by $|S|$. This is an element of $\mathbb{Q}_{\geq 0}$ that we call the mass of $P$ in $S$. By definition, we have

$$
\sum_{P \in \mathbb{R}[t]} \mathrm{m}_{S}(P)=1
$$

\footnotetext{
${ }^{3}$ Set $\zeta=e^{\frac{2 i \pi}{m}}$ for $m \geq 1$. The symmetric bilinear form $(x, y) \mapsto \operatorname{Trace}_{\mathbb{Q}(\zeta) / \mathbb{Q}}(x \bar{y})$ on the free abelian group $L=\mathbb{Z}[\zeta]$ defines a inner product on $L \otimes \mathbb{R}$. The multiplication by $\zeta$ is an isometry preserving $L$, with characteristic polynomial the $m$-th cyclotomic polynomial.
} 
(iii) For $P$ in $\mathbb{R}[t]$ a monic polynomial of degree $n$ whose complex roots are on the unit circle (e.g. $\left.P \in \mathrm{Car}_{n}\right)$, we denote by $\mathrm{c}_{P} \subset \mathrm{O}\left(\mathbb{R}^{n}\right)$ the unique conjugacy class whose characteristic polynomial is $P$.

We now discuss the problem of evaluating Formula (1.4). The main unknown, which contains all the required information about $L$ and which does not depend on $\lambda$, is of course the collection of masses $\mathrm{m}_{\mathrm{O}(L)}(P)$ for $P$ in $\mathrm{Car}_{n}$. This collection will be called the characteristic masses of $L$, or sometimes simply ${ }^{4}$ the masses of $L$, and we will go back to it later. We rather discuss first the question of evaluating, given an arbitrary polynomial $P$ as in (iii), the quantity Trace $\left(\mathrm{c}_{P} ; \mathrm{W}_{\lambda}\right)$. This question does not depend on $L$.

Evaluation of $\operatorname{Trace}\left(\mathbf{c}_{\boldsymbol{P}} ; \mathbf{W}_{\boldsymbol{\lambda}}\right)$. We will use for this the "determinantal" character formula for $\mathrm{W}_{\lambda}$ proved by Weyl in [46, Ch. VII §9]. This formula applies to arbitrary elements of $\mathrm{O}\left(\mathbb{R}^{n}\right)$, possibly of determinant -1 . We found it useful to actually use the following alternative expression proved by Koike and Terada in [33] in the spirit of the famous Jacobi-Trudi formula for the Schur polynomials in terms of elementary symmetric polynomials (see Appendix A). Write $t^{n} P(1 / t)=\sum_{i \in \mathbb{Z}}(-1)^{i} e_{i} t^{i}$ (so $e_{i}=0$ for $i<0$ or $i>n)$. Denote by $\mu_{1} \geq \mu_{2} \geq \cdots \geq \mu_{m}$ with $m=\lambda_{1}$ the partition which is dual to $\lambda$, and set $\delta_{1}=0$ and $\delta_{j}=1$ for $j>1$. Then we have the equality

$$
\operatorname{Trace}\left(\mathrm{c}_{P} ; \mathrm{W}_{\lambda}\right)=\operatorname{det}\left(e_{\mu_{i}-i+j}+\delta_{j} e_{\mu_{i}-i-j+2}\right)_{1 \leq i, j \leq m}
$$

This formula is clearly efficient when $m=\lambda_{1}$ is small, which suits well for instance the application to $\left|\mathrm{X}_{32}\right|$ mentioned in Section 1.1, as it requires all $\lambda$ 's with $\lambda_{1} \leq 4$ for $n=24$. Let us note that in this range, the use of the crude degenerate Weyl character formula as in $[9, \S 2]$ would be impracticable as the Weyl group of $\mathrm{SO}\left(\mathbb{R}^{24}\right)$ is much too big. Actually, the whole tables of invariants obtained in $[9, \S 2]$ for the subgroup of determinant 1 elements in the Weyl groups of type $\mathbf{E}_{7}, \mathbf{E}_{8}$ and $\mathbf{E}_{8} \amalg \mathbf{A}_{1}$ (with respectively $n=7,8,9)$ can be recomputed essentially instantly using rather Formula (1.5).

Determination of the characteristic masses of $\boldsymbol{L}$. This is the remaining and most important ${ }^{5}$ unknown. In dimension $n$ as large as 24 , it is impossible in general to enumerate the elements of $\mathrm{O}(L)$ with a computer, hence to naively list their characteristic polynomials. For instance when $L$ is a Niemeier lattice then the size of $\mathrm{O}(L)$ is always at least $10^{14}$, and it is

\footnotetext{
${ }^{4}$ Beware not to confuse the masses of $L$ in this sense with the mass of the genus of $L$, which traditionally appears in the study of the Minkowski-Siegel-Smith mass formula.

${ }^{5}$ It is equivalent to determine the finitely many $\mathrm{m}_{\mathrm{O}(L)}(P)$ for all $P$ in $\mathrm{Car}_{n}$, and the $\operatorname{dim} \mathrm{W}_{\lambda}^{\mathrm{O}(L)}$ for all $\lambda$, as the $\operatorname{Car}_{n} \times \Lambda$-matrix $\left(\operatorname{Trace}\left(\mathrm{c}_{P} ; \mathrm{W}_{\lambda}\right)\right)_{P, \lambda}$ has rank $\left|\operatorname{Car}_{n}\right|$ for general reasons.
} 
about $10^{30}$ for $L=\mathrm{E}_{24}$. However, those groups have of course much fewer conjugacy classes. Write

$$
\text { Conj } \mathrm{O}(L)=\left\{\mathrm{c}_{i}(L)\right\}_{i \in I}
$$

the set of conjugacy classes of $\mathrm{O}(L)$. Assuming that we know representatives of the $\mathrm{c}_{i}(L)$, as well as each $\left|\mathrm{c}_{i}(L)\right|$, then the enumeration of the characteristic polynomials of $\mathrm{O}(L)$ may become straightforward. Of course, if we do not know representatives of $\mathrm{c}_{i}(L)$, but still the trace of the latter in $\mathbb{R}^{n}$ as well as the power maps on the $c_{i}(L)$, this may similarly allow to determine the characteristic masses of $L$.

Example 1.3 (Leech lattice). Consider for instance the case where $L=$ Leech is "the" Leech lattice in $\mathbb{R}^{24}$. The group $\mathrm{O}$ (Leech) is the Conway group $\mathrm{Co}_{0}[15]$, also denoted $2 . \mathrm{Co}_{1}$ in the $\mathbb{A} \mathbb{L} \mathbb{A} \mathbb{S}[16$, p. 180]. The character of its natural representation on $\mathbb{R}^{24}$ is the character $\chi_{102}$ in the table loc. cit. This character, as well as Newton's relations and the power maps of the $\mathbb{A} \mathbb{L} \mathbb{A} \mathbb{S}$ (implemented in GAP [26]), allow to compute the characteristic polynomial of each conjugacy class in $\mathrm{O}$ (Leech), hence the characteristic masses of Leech: they are gathered in Table C.5. Note that despite the huge order $\simeq 8 \cdot 10^{18}$ of $\mathrm{O}$ (Leech), this group only has 167 conjugacy classes, and 160 distinct characteristic polynomials. This is actually the minimum for a Niemeier lattice, and makes the table above printable. An interesting consequence of this computation is the observation

$$
\frac{1}{\mid \mathrm{O}(\text { Leech }) \mid} \sum_{g \in \mathrm{O}(\text { Leech })} \operatorname{det}\left(t \mathrm{id}_{24}-g\right)=t^{24}+t^{16}+t^{12}+t^{8}+1 .
$$

This asserts the existence of a line of $\mathrm{O}($ Leech)-equivariant alternating $g$ multilinear form Leech $^{g} \longrightarrow \mathbb{Z}$ for each $g$ in $\{8,12,16,24\}$. We refer to [10] for a study of these forms and of the weight 13 pluriharmonic Siegel theta series for $\operatorname{Sp}_{2 g}(\mathbb{Z})$ that they allow to construct. The results of this paper suggest several other intriguing constructions to study in the same spirit, for instance whenever a 1 appears as a dimension for $\mathrm{M}_{\mathrm{W}_{\lambda}}\left(\mathrm{O}_{24}\right)$ in Table C.6 (the case discussed here corresponding to $\lambda=\emptyset, 1^{8}$ and $1^{12}$ ).

1.4. Algorithms for computing characteristic masses. Let us give now a first algorithm, called Algorithm $A$ in the sequel, which takes as input the Gram matrix $G$ of some $\mathbb{Z}$-basis of $L$ and returns for each conjugacy class $\mathrm{c}_{i}(L)$ some representative and its cardinality $\left|\mathrm{c}_{i}(L)\right|$, hence in particular the characteristic masses of $L$. The idea, certainly classical in computational group theory, is to:

A1. Apply the Plesken-Souvignier algorithm [40] to $G$ (implemented e.g. as qfauto $(G)$ in PARI/GP [39]) to obtain a set $\mathcal{G}$ of generators of $\mathrm{O}(L)$, 
A2. Choose a (small) finite subset $\mathcal{S} \subset L$ stable under $\mathrm{O}(L)$, generating $L \otimes \mathbb{R}$, and view $\mathrm{O}(L)$ as the subgroup of permutations of $\mathcal{S}$ generated by $\mathcal{G}$,

A3. Apply permutation groups algorithms implemented in GAP (such as [30]) to deduce cardinality and representatives of the conjugacy classes of $\mathrm{O}(L)$.

A canonical choice of $\mathcal{S}$ is the following: for any lattice $L$ set (inductively) $S(L)=M(L) \amalg S\left(L^{\prime}\right)$ where $M(L)$ is the subset of elements of $L$ with minimal nonzero length, and where $L^{\prime}$ is the orthogonal of $M(L)$ in $L$. The choice $\mathcal{S}=S(L)$ has proved efficient enough for us in practice. We will say more about a PARI/GP implementation of the whole algorithm later, when discussing an improvement of it: see Section 4.3.

Algorithm $A$ is very efficient in small dimension. For instance, when $L$ is a root lattice of type $\mathbf{E}_{6}, \mathbf{E}_{7}$ or $\mathbf{E}_{8}$, it returns the characteristic masses of $L$ in a few seconds only. ${ }^{6}$ It turns out that it is still terminates for most of the even lattices of determinant $\leq 2$ and dimension $\leq 25$, with running time varying from a few minutes to a few days in dimensions 23, 24 and 25 when terminates. For instance, in the case $L=$ Leech it allows to re-compute Table C.5 from scratch, without relying at all on the $\mathbb{A} \mathbb{T} L \mathbb{A} S$ : it requires about 3 minutes for step A1, nothing for A2, and 42 minutes for A3. On the other hand, it does not terminate for instance on our computer for lattices $L$ in $\mathcal{L}_{25}$ with root system ${ }^{7} \mathbf{A}_{1} \mathbf{D}_{4} 2 \mathbf{D}_{6} \mathbf{D}_{8}$ or $\mathbf{A}_{1} \mathbf{D}_{6} \mathbf{D}_{8} \mathbf{D}_{10}$ (memory issue). Algorithm $\mathbf{A}$ is typically very slow (and memory consuming) if either $L$ has too many vectors $v$ of length $v \cdot v=G_{i, i}$ for some $i=1, \ldots, n$, because of step $\mathrm{A} 1$, or if $\mathrm{O}(L)$ has too many conjugacy classes, because of step A3. It is also quite sensitive to the choice of Gram matrix $G$ of $L$ in step A1.

In Section 4, we will explain a significant improvement of Algorithm A when $L$ has a non trivial root system. The basic idea of this Algorithm B is to first write

$$
\mathrm{O}(L)=\mathrm{W}(R) \rtimes \mathrm{O}(L)_{\rho}
$$

where $R$ is the root system of $L, \mathrm{~W}(R)$ its Weyl group, $\rho$ a Weyl vector of $R$ and $\mathrm{O}(L)_{\rho}$ the stabilizer of $\rho$ in $\mathrm{O}(L)$. As we shall see, we may actually reduce the computation of the characteristic masses of $L$ to that of representatives $\gamma_{j}$, and sizes, of the conjugacy classes of the smaller group $\mathrm{O}(L)_{\rho}$, an information which can be obtained by replacing $\mathrm{O}(L)$ with $\mathrm{O}(L)_{\rho}$ in steps A1 and A3 of Algorithm A. There are two ingredients for this reduction. The first is the determination, for each rank $r$ irreducible root system

\footnotetext{
${ }^{6}$ All the computations in this paper have been made on a processor Intel(R) Xeon(R) CPU E5-2650 v4 @ 2.20GHz with 65 GB of memory. Nevertheless, all the computations involving either Algorithm B, or Algorithm A in small dimension, are equally efficient on our personal computer (processor 1,8 GHz Intel Core i5 with 8 GB of memory).

${ }^{7}$ For $n \leq 25$, it follows from the classification of $\mathrm{X}_{n}$ recalled in Section 1.1 that two lattices in $\mathcal{L}_{n}$ are isometric if, and only if, they have isomorphic root systems.
} 
$R^{\prime}$ of type ADE, of the map $\mathrm{m}_{S}: \operatorname{Car}_{r} \rightarrow \mathbb{Q}$, where $S$ is any coset of $\mathrm{W}\left(R^{\prime}\right)$ in the full isometry group $\mathrm{O}\left(R^{\prime}\right)$ of the root system $R^{\prime}$ : see Section 3 for this step (which does not depend on $L$ ). The second is the determination, for each $j$, of the conjugacy class of $\gamma_{j}$ viewed as an element of the automorphism group of the Dynkin diagram of $R$. See Section 4 for a detailed discussion of Algorithm B and of its implementation.

Remark 1.5 (Generalizations). In this paper, we use a restricted notion of root which suits well our applications to the lattices in $\mathcal{L}_{n}$. A minor modification of Algorithm B allows to consider the most general roots, namely the elements $\alpha$ of a lattice $L$ such that the orthogonal symmetry about $\alpha$ preserves $L$. In a different direction, it would be useful to extend the algorithms above to the context of hermitian or quaternionic positive definite lattices, possibly over totally real number fields, using the theory of complex or quaternionic reflection groups (see e.g. [13, 14]). That should help extending to higher ranks and weights the computations of dimension spaces of automorphic forms for definite unitary groups (hermitian or quaternionic) started in the literature (e.g. in $[19,20,27,35,36])$.

1.6. Main results. Using Algorithm $B$, it only takes a few seconds to the computer to compute all the characteristic masses of each Niemeier lattices with roots, except in the case (trivial anyway) of $\mathrm{E}_{24}$ for which the PleskenSouvignier algorithm needs about 2 minutes. It is equally efficient in any dimension $\leq 25$ : the characteristic masses are computed in a few seconds, except for ten lattices (in dimension 23 or 25) for which it requires less than 5 minutes, and for the lattice $\mathrm{A}_{1} \oplus$ Leech in $\mathcal{L}_{25}$ (about 35 minutes). We refer to the homepage [6] for the gram matrices we used in our computations. Our main result is then the following.

\section{Theorem 1.}

(i) Assume $n \leq 25$. The characteristic masses of all $L \in \mathcal{L}_{n}$ are those given $^{8}$ in $[6]$.

(ii) The nonzero values of $\operatorname{dim} \mathrm{M}_{\lambda}\left(\mathrm{O}_{24}\right)$ for $\lambda_{1} \leq 3$ are given in Table C.6.

Table C.6 is deduced from assertion (i) for $n=24$ using observation (b) and Formulas (1.4) \& (1.5). This step is very efficient: once the masses in (i) are computed, it takes only 5 minutes about to produce this table. The format of the table is as follows. The notation $n_{1}^{m_{1}} \ldots n_{r}^{m_{r}}$ for a partition $\lambda$ means that the diagram of $\lambda$ has exactly $m_{i}$ rows of size $n_{i}$ for $i=$ $1, \ldots, r$, and no other row. Set $\mathrm{d}_{\lambda}=\operatorname{dim} \mathrm{M}_{\mathrm{W}_{\lambda}}\left(\mathrm{O}_{24}\right)$ and denote by $\operatorname{ass}(\lambda)$ the associate of $\lambda$ (see Section A). The column dim gives the integer $d_{\lambda}$ in

\footnotetext{
8 They cannot be printed here: there are 53204 polynomials $P$ with $\mathrm{m}_{\mathrm{O}(L)}(P) \neq 0$ for some $L$ in $\mathcal{L}_{24}$, that is about half $\left|\operatorname{Car}_{24}\right|$.
} 
the case $\lambda=\operatorname{ass}(\lambda)$, and the two integers $d_{\lambda}: d_{\text {ass }(\lambda)}$ otherwise. See [6] for more extensive tables, including for instance all $\lambda$ with $\lambda_{1}=4$ and arbitray $n \leq 25$.

Remark 1.7. Fix $L$ in $\mathcal{L}_{n}, \gamma$ in $\mathrm{O}(L)$, and write $\operatorname{det}(t-\gamma)=(t-1)^{a} \times$ $(t+1)^{b} Q(t)$ with $Q$ in $\mathbb{Z}[t]$ and $Q(-1) Q(1) \neq 0$. Then Proposition 3.7 in [11], generalizing a result of Gross and McMullen [28], shows ${ }^{9}$ that for $a=0$ (resp. $b=0$ ) the integer $Q(1)$ (resp. $Q(-1)$ ) is a square. This constraint is in agreement with our computations. The question of the existence of an even unimodular lattice having an isometry of given characteristic polynomial has been studied by several authors: see e.g. [1, 2, 28, 37].

1.8. A direct computation in the case of Niemeier lattices. In Section 5, we will explain an alternative (and human) computation of the characteristic masses of Niemeier lattices. By the results of Section 3, we are left to determine, for each Niemeier lattice $L$ with non-empty root system $R$, the $\mathrm{G}(R)$-conjugacy classes of the elements of the subgroup $\mathrm{O}(L) / \mathrm{W}(R)$ of $\mathrm{G}(R)$, where $\mathrm{G}(R)$ is the automorphism group of the Dynkin diagram of $R$. We do so using a tedious case by case analysis.

We found it useful to gather first in Section 2 some elementary results about the hyperoctahedral group $\mathrm{H}_{n}=\{ \pm 1\}^{n} \rtimes \mathrm{S}_{n}$. This group is both a typical direct summand of the $\mathrm{G}(R)$ above, and closely related to the Weyl groups of type $\mathbf{D}_{n}$ studied in Section 3.2. In particular, we introduce and characterize directly in Section 2.5 and Section 2.7 a few specific subgroups of $\mathrm{H}_{n}$ that will play a role in the analysis of Niemeier lattices in Section 5 .

Although more interesting (at least to us) from a mathematical point of view, it will be eventually clear that this nonautomatized method is too complicated to be used systematically: it would even require some work to attack the dimensions 23 and 25 along the same lines. Nevertheless, it provides an important check that the masses returned by the implementation of our algorithms are correct.

1.9. General notations and conventions. In this paper, all group actions will be on the left. We denote by $|X|$ the cardinality of the set $X$. For $n \geq 1$ an integer, we denote by $S_{n}$ the symmetric group on $\{1, \ldots, n\}$, by $\mathrm{Alt}_{n} \subset \mathrm{S}_{n}$ the alternating subgroup, and we set $\mathbb{Z} / n:=\mathbb{Z} / n \mathbb{Z}$.

If $V$ is an Euclidean space, we usually denote by $x \cdot y$ its inner product, with associated quadratic form $\mathrm{q}: V \rightarrow \mathbb{R}$ defined by $\mathrm{q}(x)=\frac{x \cdot x}{2}$. A lattice in $V$ is a subgroup generated by a basis of $V$, or equivalently, a discrete subgroup $L$ with finite covolume, denoted covol $L$.

If $L$ is a lattice in the Euclidean space $V$, its dual lattice is the lattice $L^{\sharp}$ defined as $\{v \in V \mid v \cdot x \in \mathbb{Z}, \forall x \in L\}$. We say that $L$ is integral (resp. even) if we have $L \subset L^{\sharp}$ (resp. $\left.\mathrm{q}(L) \subset \mathbb{Z}\right)$. An even lattice is integral. If $L$ is

\footnotetext{
${ }^{9}$ If $\operatorname{det} \gamma=-1$ (so $b$ is odd) and $a=0$ (so $n$ is odd), apply that proposition to $-\gamma$.
} 
integral, we have $(\operatorname{covol} L)^{2}=\left|L^{\sharp} / L\right|$. This integer is also the determinant $\operatorname{det} L$ of the $\operatorname{Gram}$ matrix $\operatorname{Gram}(e)=\left(e_{i} \cdot e_{j}\right)_{1 \leq i, j \leq n}$ of any $\mathbb{Z}$-basis $e=$ $\left(e_{1}, \ldots, e_{n}\right)$ of $L$. The orthogonal group of $L$ is the finite group $\mathrm{O}(L)=$ $\{\gamma \in \mathrm{O}(V), \gamma(L)=L\}$.

\section{Preliminaries on the hyperoctahedral groups}

2.1. The hyperoctahedral group. Let $n \geq 1$ be an integer. The symmetric group $\mathrm{S}_{n}$ on the set $\{1, \ldots, n\}$ acts on the elementary abelian 2-group $\{ \pm 1\}^{n}$ by permuting coordinates. The hyperoctahedral group on $n$ letters is defined as the semi-direct product

$$
\mathrm{H}_{n}=\{ \pm 1\}^{n} \rtimes \mathrm{S}_{n} .
$$

Equivalently, $\mathrm{H}_{n}$ is the wreath product $\{ \pm 1\}<S_{n}$. It is isomorphic to several familiar groups: the Weyl group of a root system of type $\mathbf{B}_{n}$ or $\mathbf{C}_{n}$, the subgroup of monomial matrices in $\mathrm{GL}_{n}(\mathbb{Z})$, the orthogonal group of the standard unimodular lattice $\mathrm{I}_{n}$, the subgroup of the symmetric group on $\{ \pm 1, \pm 2, \ldots, \pm n\}$ of permutations $\sigma$ with $\sigma(-i)=-\sigma(i)$ for all $i$, etc.

In this paper, we will encounter $\mathrm{H}_{n}$ first when discussing $\mathrm{O}\left(\mathrm{D}_{n}\right)$ and again when studying automorphism groups of isotypic root systems. Certain subgroups of the hyperoctahedral groups will play a role in the study of Niemeier lattices. Here is an example of an interesting subgroup that will occur in the case $n=4$. We denote by $\pi: \mathrm{H}_{n} \rightarrow \mathrm{S}_{n}$ the canonical projection.

Example 2.2. The group $\mathrm{GL}_{2}(\mathbb{Z} / 3)$ acts on the 8-elements set $(\mathbb{Z} / 3)^{2}-\{0\}$ by permuting the 4 disjoint pairs of the form $\{v,-v\}$. By the universal property of wreath products, the choice of elements $v_{1}, v_{2}, v_{3}, v_{4}$ such that $(\mathbb{Z} / 3)^{2}-\{0\}=\coprod_{i}\left\{v_{i},-v_{i}\right\}$ defines an embedding $\iota: \mathrm{GL}_{2}(\mathbb{Z} / 3) \rightarrow \mathrm{H}_{4}(\mathrm{a}$ different choice leading to an $\mathrm{H}_{4}$-conjugate embedding). We have $\iota\left(-\mathrm{Id}_{2}\right)=$ -1 ; the morphism $\pi \circ \iota$ has kernel $\pm \mathrm{Id}_{2}$ and induces "the" exceptional isomorphism $\mathrm{PGL}_{2}(\mathbb{Z} / 3) \simeq \mathrm{S}_{4}$. The restriction of $\pi \circ \iota$ to the stabilizer of $v_{i}$ in $\mathrm{GL}_{2}(\mathbb{Z} / 3)$ is an isomorphism onto the stabilizer $\left(\simeq \mathrm{S}_{3}\right)$ of $i$ in $\{1,2,3,4\}$.

We end this paragraph with a few notations and remarks about the basic structure of $\mathrm{H}_{n}$. We denote by $\varepsilon_{i}$ the element of $\{ \pm 1\}^{n}$ whose $j^{\text {th }}$-component is 1 for $j \neq i$ and -1 for $j=i$. The center of $\mathrm{H}_{n}$ is generated by the element $-1=\prod_{i=1}^{n} \varepsilon_{i}$. The signature $\epsilon: \mathrm{S}_{n} \rightarrow\{ \pm 1\}$, composed with the natural projection $\pi: \mathrm{H}_{n} \rightarrow \mathrm{S}_{n}$, defines a morphism $\mathrm{H}_{n} \rightarrow\{ \pm 1\}$ that we will still denote by $\epsilon$. Another important morphism $\mathrm{s}: \mathrm{H}_{n} \rightarrow\{ \pm 1\}$ is defined by

$$
\mathrm{s}(v \sigma)=\prod_{i=1}^{n} v_{i}, \quad \text { for all } \sigma \in \mathrm{S}_{n} \text { and } v=\left(v_{i}\right) \in\{ \pm 1\}^{n} \text {. }
$$

The product character $\epsilon \mathrm{s}$ coincides with the determinant when we view $\mathrm{H}_{n}$ as a the subgroup of monomial matrices in $\mathrm{GL}_{n}(\mathbb{Z})$. We now recall the classical description of the conjugacy classes of $\mathrm{H}_{n}$ (see e.g. [5, Prop. 25]). 
2.3. Conjugacy classes of $\mathbf{H}_{\boldsymbol{n}}$. Let $\Sigma$ be a nonempty subset of $\{1, \ldots, n\}$. A cycle in $\mathrm{H}_{n}$ with support $\Sigma$ is an element of the form $h=v c$, where $c \in \mathrm{S}_{n}$ permutes transitively the elements of $\Sigma$ and fixes its complement, and where $v=\left(v_{i}\right) \in\{ \pm 1\}^{n}$ satisfies $v_{i}=1$ for $i \notin \Sigma$. Such a cycle has a length $1(h)$ defined as $|\Sigma|$, and a sign $\mathrm{s}(h)$ (an element in $\{ \pm 1\}$ ). This sign is also the $i$-th coordinate of $h^{1(h)}$ for any $i$ in $\Sigma$, and $l(h)$ is the order of $c$. One easily checks that two cycles are conjugate in $\mathrm{H}_{n}$ if, and only if, they have the same length and the same sign.

Just as for $\mathrm{S}_{n}$, any element $h$ of $\mathrm{H}_{n}$ may be written as a product of cycles $h_{i}$ with disjoint supports, this decomposition being unique up to permutation of those cycles. The sum of the lengths of the cycles $h_{i}$ with $\mathrm{s}\left(h_{i}\right)=1$ (resp. $\mathrm{s}\left(h_{i}\right)=-1$ ) is an integer denoted $\mathrm{n}_{+}(h)$ (resp $\mathrm{n}_{-}(h)$ ); the collection of the length $\mathrm{l}\left(h_{i}\right)$ of those $h_{i}$ defines a integer partition of $\mathrm{n}_{+}(h)$ (resp. $\mathrm{n}_{-}(h)$ ) that we denote by $\mathrm{p}_{+}(h)$ (resp. $\mathrm{p}_{-}(h)$ ). We have $\mathrm{n}_{+}(h)+$ $\mathrm{n}_{-}(h)=n$. The type of $h$ is defined as the couple of integer partitions $\left(\mathrm{p}_{+}(h), \mathrm{p}_{-}(h)\right)$. Two elements of $\mathrm{H}_{n}$ are conjugate if, and only if, they have the same type.

In the sequel, we will have to determine the type of all the elements of certain specific subgroups $G \subset \mathrm{H}_{n}$. For instance, when $G$ is the group $\iota\left(\mathrm{GL}_{2}(\mathbb{Z} / 3)\right)$ of Example 2.2, this information is given in Table 2.1, the row size giving the number of elements of the corresponding type divided by $|G|$ :

TABLE 2.1. The $\mathrm{H}_{4}$-conjugacy classes of the elements of $\mathrm{GL}_{2}(\mathbb{Z} / 3)$.

\begin{tabular}{c|c|c|c|c|c|c|c} 
type & $1^{4}$ & $1^{4}$ & 112 & $2^{2}$ & 13 & 13 & 4 \\
\hline size & $1 / 48$ & $1 / 48$ & $1 / 4$ & $1 / 8$ & $1 / 6$ & $1 / 6$ & $1 / 4$
\end{tabular}

In this table, and in others that we will give later, we use standard notations for partitions, and print $\mathrm{p}_{+}$in black and $\mathrm{p}_{-}$in cyan. So the sequence of symbols $1^{a_{1}} 1^{b_{1}} 2^{a_{2}} 2^{b_{2}} \ldots i^{a_{i}} i^{b_{i}} \ldots$ stands for the couple $\left(p_{+}, p_{-}\right)$where $p_{+}$is the partition of $\sum_{i} a_{i}$ in $a_{1}$ times $1, a_{2}$ times 2 , and so on, and $p_{-}$ is the partition of $\sum_{i} b_{i}$ in $b_{1}$ times $1, b_{2}$ times 2 , and so on. The symbol " $i$ " (resp. " $i$ ") is omitted for $m=0$, and replaced by " $i$ " (resp. " $i ")$ for $m=1$.

Remark 2.4. Table 2.1 is easily deduced from the conjugacy classes of $\mathrm{GL}_{2}(\mathbb{Z} / 3)$. To fix ideas, define the embedding $\iota$ in Example 2.2 by choosing $v_{1}, v_{2}, v_{3}$ and $v_{4}$ to be respectively $\left[\begin{array}{l}0 \\ 1\end{array}\right],\left[\begin{array}{l}1 \\ 0\end{array}\right],\left[\begin{array}{l}1 \\ 1\end{array}\right]$ and $\left[\begin{array}{c}1 \\ -1\end{array}\right]$. Then the images under $\iota$ of the elements $\pm \mathrm{I}_{2},\left[\begin{array}{cc}1 & 0 \\ 0 & -1\end{array}\right],\left[\begin{array}{cc}0 & -1 \\ 1 & 0\end{array}\right], \pm\left[\begin{array}{ll}1 & 1 \\ 0 & 1\end{array}\right]$ and $\pm\left[\begin{array}{ll}0 & 1 \\ 1 & 1\end{array}\right]$ of $\mathrm{GL}_{2}(\mathbb{Z} / 3)$ are respectively $\pm 1, \varepsilon_{1}(34), \varepsilon_{2}(12) \varepsilon_{4}(34), \pm \varepsilon_{1} \varepsilon_{4}(134)$ and $\pm \varepsilon_{2}(1342)$.

2.5. Digression: subgroups of certain wreath products. Let $G$ be a group, $X$ a set equipped with a transitive action of $G$, and $A$ an abelian 
group. The group $G$ acts in a natural way on the abelian group $A^{X}$ of all functions $X \rightarrow A$, so we can form the semi-direct product $H:=A^{X} \rtimes G$.

We denote by $\pi: H \rightarrow G$ the canonical projection, with kernel ker $\pi=$ $A^{X}$. We have a "diagonal" map $\delta: A \rightarrow A^{X}$, defined by $\delta(a)(x)=a$ for all $a$ in $A$ and $x$ in $X$. This map $\delta$ is an embedding of $G$-modules if we view the source $A$ as a trivial $G$-module: the image of $\delta$ is a central subgroup of $H$. Our aim in this paragraph is to study:

- the set $\mathcal{C}$ of subgroups $C \subset H$ with $\pi(C)=G$ and $\operatorname{ker} \pi \cap C=1$,

- the set $\mathcal{G}$ of subgroups $\widetilde{G} \subset H$ with $\pi(\widetilde{G})=G$ and $\operatorname{ker} \pi \cap \widetilde{G}=\delta(A)$.

The group $H$ acts both on $\mathcal{C}$ and $\mathcal{G}$ by conjugation. We start with two simple observations:

- For any group morphism $\chi: G \rightarrow A$, the set $G^{\chi}:=\{\delta(\chi(g)) \cdot g, g \in$ $G\}$ is a subgroup of $H$ isomorphic to $G$, and $G^{\chi}$ is an element of $\mathcal{C}$.

- There is a natural map $\mathrm{c}_{2}: \mathcal{G} \rightarrow \mathrm{H}^{2}(G, A)$, sending $\widetilde{G}$ in $\mathcal{G}$ to the equivalence class of the central extension $1 \rightarrow A \stackrel{\delta}{\rightarrow} \widetilde{G} \stackrel{\pi}{\rightarrow} G \rightarrow 1$. Two elements of $\mathcal{G}$ which are $H$-conjugate are also $A^{X}$-conjugate, hence define the same class in $\mathrm{H}^{2}(G, A)$.

We fix some $x \in X$ and denote by $G_{x} \subset G$ the isotropy group of $x$. For each integer $m \geq 0$, we denote by $\mathrm{r}_{m}: \mathrm{H}^{m}(G, A) \longrightarrow \mathrm{H}^{m}\left(G_{x}, A\right)$ the usual restriction map on the cohomology groups of the trivial $G$-module $A$.

\section{Proposition 2.6.}

(i) For $\chi, \chi^{\prime} \in \operatorname{Hom}(G, A)$, the subgroups $G^{\chi}$ and $G^{\chi^{\prime}}$ of $H$ are conjugate if, and only if, $\chi$ and $\chi^{\prime}$ coincide on $G_{x}$.

(ii) If $\mathrm{r}_{1}$ is surjective then any subgroup $C \in \mathcal{C}$ is conjugate to $G^{\chi}$ for some $\chi \in \operatorname{Hom}(G, A)$.

(iii) If $\mathrm{r}_{1}$ is surjective then the map $\mathrm{c}_{2}: H \backslash \mathcal{G} \rightarrow \mathrm{H}^{2}(G, A)$ is injective, and its image is the subgroup ker $_{2}$ of extensions which split over $G_{x}$.

Proof. We shall use twice the following classical facts. Let $\Gamma$ be a group acting on an abelian group $V$ and denote by $\pi: V \rtimes \Gamma \rightarrow \Gamma$ the natural projection. Let $\mathcal{K}$ be the set subgroups $K \subset V \rtimes \Gamma$ with $\pi(K)=\Gamma$ and $\operatorname{ker} \pi \cap V=1$. Any $K \in \mathcal{K}$ has the form $\{s(\gamma) \gamma, \gamma \in \Gamma\}$ for a unique 1cocyle $s \in \mathrm{Z}^{1}(\Gamma, V)$, that we denote $\mathrm{s}_{K}$. The map $K \mapsto \mathrm{s}_{K}, \mathcal{K} \rightarrow \mathrm{Z}^{1}(\Gamma, V)$, is bijective; two elements $K, K^{\prime}$ in $\mathcal{K}$ are conjugate by an element of $V$ if, and only if, $\mathrm{s}_{K}$ and $\mathrm{s}_{K^{\prime}}$ have the same class in $\mathrm{H}^{1}(\Gamma, V)$. Last but not least, note that $K, K^{\prime}$ in $\mathcal{K}$ are conjugate by an element of $V$ if, and only if, they are conjugate in $V \rtimes \Gamma$ : if we have $K^{\prime}=g K g^{-1}$ with $g \in V \rtimes \Gamma$, we may write $g=v k$ with $v \in V$ and $k \in K$, and we have $K^{\prime}=v K v^{-1}$.

We apply this first to $\Gamma=G$ and $V=A^{X}$. The map $\operatorname{Hom}(G, A)=$ $\mathrm{Z}^{1}(G, A) \rightarrow \mathrm{Z}^{1}\left(G, A^{X}\right)$ defined by $\delta$ sends $\chi$ to the 1 -cocycle defining $G^{\chi}$. 
The choice of $x \in X$ identifies the $G$-module $A^{X}$ with the co-induced module of the trivial $G_{x}$-module $A$ to $G$. By Shapiro's lemma, we obtain for each integer $m \geq 0$ a natural isomorphism $\operatorname{sh}: \mathrm{H}^{m}\left(G, A^{X}\right) \stackrel{\sim}{\longrightarrow} \mathrm{H}^{m}\left(G_{x}, A\right)$. Concretely, if $f: G^{m} \rightarrow A^{X}$ is an $m$-cocycle, then $\operatorname{sh}(f)$ is the class of the $m$-cocycle $f^{\prime}: G_{x}^{m} \rightarrow A$ defined by $f^{\prime}\left(g_{1}, \ldots, g_{m}\right)=f\left(g_{1}, \ldots, g_{m}\right)(x)$. It follows that the composition of the maps

$$
\mathrm{H}^{m}(G, A) \stackrel{\mathrm{H}^{m}(\delta)}{\longrightarrow} \mathrm{H}^{m}\left(G, A^{X}\right) \stackrel{\text { sh }}{\sim} \mathrm{H}^{m}\left(G_{x}, A\right)
$$

coincides with the map $\mathrm{r}_{m}$. For $m=1$, this proves assertions (i) and (ii).

Let us prove assertion (iii). Let $Q$ be the cokernel of $\delta$. By applying the first paragraph above to $\Gamma=G$ and $V=Q$, we obtain a natural bijection $\mathrm{c}_{1}: H \backslash \mathcal{G} \stackrel{\sim}{\longrightarrow} \mathrm{H}^{1}(G, Q)$. The long exact sequence of cohomology groups associated to $0 \rightarrow A \stackrel{\delta}{\rightarrow} A^{X} \rightarrow Q \rightarrow 0$ contains a piece of the form

$$
\mathrm{H}^{1}(G, A) \stackrel{\mathrm{H}^{1}(\delta)}{\longrightarrow} \mathrm{H}^{1}\left(G, A^{X}\right) \longrightarrow \mathrm{H}^{1}(G, Q) \stackrel{\eta}{\longrightarrow} \mathrm{H}^{2}(G, A) \stackrel{\mathrm{H}^{2}(\delta)}{\longrightarrow} \mathrm{H}^{2}\left(G, A^{X}\right) .
$$

By the second paragraph, the kernel of $\eta$ is isomorphic to the cokernel of $\mathrm{r}_{1}$, and the image of $\eta$ is the kernel of $\mathrm{r}_{2}$. As it is straightforward to check from the definition of $c_{2}$ that we have $\eta \circ c_{1}=c_{2}$, this concludes the proof of assertion (iii).

2.7. Applications to $\mathbf{H}_{n}$. The group $\mathrm{H}_{n}$ is of course the special case of the construction of Section 2.5 with $G=\mathrm{S}_{n}, X=\{1, \ldots, n\}$ and $A=\{ \pm 1\}$ (multiplicative group). The signature $\epsilon$ gives rise to the subgroup $\mathrm{S}_{n}^{\epsilon}$ of $\mathrm{H}_{n}$ whose elements have the form $\epsilon(\sigma) \sigma, \sigma \in \mathrm{S}_{n}$. For any transposition $\tau$ in $\mathrm{S}_{n}$ we have $\mathrm{n}_{-}(\epsilon(\tau) \tau)=n-2$, whereas $\mathrm{n}_{-}(\sigma)=0$ for all $\sigma$ in $\mathrm{S}_{n}$ : this shows that $\mathrm{S}_{n}^{\epsilon}$ is not conjugate to $\mathrm{S}_{n}$ in $\mathrm{H}_{n}$ for $n>2$ (a fact which also follows from assertion (i) below).

\section{Proposition 2.8.}

(i) Let $G$ be a subgroup of $\mathrm{H}_{n}$ of order $n$ ! with $\pi(G)=\mathrm{S}_{n}$. Then $G$ is either conjugate to $\mathrm{S}_{n}$ or to $\mathrm{S}_{n}^{\epsilon}$. Moreover, $\mathrm{S}_{n}$ and $\mathrm{S}_{n}^{\epsilon}$ are conjugate in $\mathrm{H}_{n}$ if, and only if, we have $n \leq 2$.

(ii) Let $G$ be a subgroup of $\mathrm{H}_{n}$ of order $2 n$ ! with $\pi(G)=\mathrm{S}_{n}$. Then -1 is in $G$ and exactly one of the following properties holds:

(a) $G$ is conjugate to $\{ \pm 1\} \cdot \mathrm{S}_{n}$,

(b) $n=2$ and $G \simeq \mathbb{Z} / 4$,

(c) $n=4$ and $G$ is conjugate to the group $\mathrm{GL}_{2}(\mathbb{Z} / 3)$ embedded in $\mathrm{H}_{4}$ as in Example 2.2.

Proof. Note first that in case (ii), $\{ \pm 1\}^{n} \cap G$ is a normal subgroup of order 2 of $G$, hence it is central and generated by -1 by the assumption $\pi(G)=\mathrm{S}_{n}$.

The stabilizer of $n$ in $\mathrm{S}_{n}$ is naturally identified with $\mathrm{S}_{n-1}$, with the convention $\mathrm{S}_{0}=1$. The signature $\epsilon$ is a generator of $\mathrm{H}^{1}\left(\mathrm{~S}_{n},\{ \pm 1\}\right)$, so the 
restriction map $\mathrm{H}^{1}\left(\mathrm{~S}_{n},\{ \pm 1\}\right) \rightarrow \mathrm{H}^{1}\left(\mathrm{~S}_{n-1},\{ \pm 1\}\right)$ is clearly surjective, and bijective for $n \neq 2$. Moreover, we know form Schur that the restriction map $\mathrm{r}_{2}: \mathrm{H}^{2}\left(\mathrm{~S}_{n},\{ \pm 1\}\right) \rightarrow \mathrm{H}^{2}\left(\mathrm{~S}_{n-1},\{ \pm 1\}\right)$ is surjective as well for all $n>1$, and that the dimension of the $\mathbb{Z} / 2$-vector space $\mathrm{H}^{2}\left(\mathrm{~S}_{n},\{ \pm 1\}\right)$ is 2 for $n \geq 4$, 1 for $n=3$ and 2, and 0 for $n=1$ [41]. The kernel of $\mathrm{r}_{2}$ is thus 0 for $n \neq 2,4$, isomorphic to $\mathbb{Z} / 2$ otherwise. We conclude by Proposition 2.6 and Example 2.2.

Remark 2.9. The natural map $\mathrm{H}^{i}\left(\mathrm{Alt}_{4}, \mathbb{Z} / 2\right) \rightarrow \mathrm{H}^{i}\left(\mathrm{Alt}_{3}, \mathbb{Z} / 2\right)$ is $0 \rightarrow 0$ for $i=1$ and $\mathbb{Z} / 2 \rightarrow 0$ for $i=2$. By Proposition 2.6(iii), there is thus a unique conjugacy class of nonsplit central extensions of Alt 4 by $\{ \pm 1\}$ in $\mathrm{H}_{4}$ (or in $\left.\{ \pm 1\}^{4} \rtimes \mathrm{Alt}_{4}\right)$. As $\mathrm{Alt}_{4}$ does not embed in $\mathrm{GL}_{2}(\mathbb{Z} / 3)$, one such extension is the inverse image of $\mathrm{Alt}_{4}$ in the extension described in Example 2.2.

We now give another example. As is well-known, the group $S_{5}$ has a unique isomorphism class of transitive actions on the set $\{1, \ldots, 6\}$, obtained from the conjugation action on its 6 subgroups of order 5 . We fix such an action and consider the associated semi-direct product $\{ \pm 1\}^{6} \rtimes \mathrm{S}_{5}$, as in Section 2.5. We have a defined loc. cit. a set $\mathcal{G}$ of subgroups of $\{ \pm 1\}^{6} \rtimes \mathrm{S}_{5}$ which are central extensions of $S_{5}$ by $\{ \pm 1\}$.

Proposition 2.10. The set $\mathcal{G}$ is the disjoint union of two conjugacy classes: the one of the split extension $\{ \pm 1\} \cdot \mathrm{S}_{5}$, and another one consisting of nonsplit extensions which are split over the alternating subgroup $\mathrm{Alt}_{5}$ of $\mathrm{S}_{5}$.

Proof. Let $N \subset \mathrm{S}_{5}$ be the normalizer of the subgroup $S=\langle(12345)\rangle$. Then $N$ is the semi-direct product of $\langle(2354)\rangle \simeq \mathbb{Z} / 4$ by $S \simeq \mathbb{Z} / 5$, so we have $\mathrm{H}^{i}(N, \mathbb{Z} / 2) \simeq \mathbb{Z} / 2$ for each $i \geq 0$ and the restriction map $\mathrm{H}^{1}\left(\mathrm{~S}_{5}, \mathbb{Z} / 2\right) \rightarrow$ $\mathrm{H}^{1}(N, \mathbb{Z} / 2)$ is an isomorphism. We observe from the presentation given by Schur of the two Schur-covers of $\mathrm{S}_{5}$ that they are non split over the subgroups of $\mathrm{S}_{5}$ containing a double transposition, such as $N$ or $\mathrm{Alt}_{5}$. This implies that the kernel of the restriction map $\mathrm{H}^{2}\left(\mathrm{~S}_{5}, \mathbb{Z} / 2\right) \rightarrow \mathrm{H}^{2}(N, \mathbb{Z} / 2)$ is generated by the remaining nonzero class in $\mathrm{H}^{2}\left(\mathrm{~S}_{5}, \mathbb{Z} / 2\right)$, namely the one

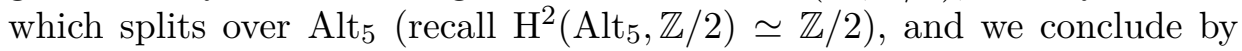
Proposition 2.6.

A homomorphism $\mathrm{S}_{5} \rightarrow \mathrm{S}_{6}$ as above can alternatively be constructed from the natural action of $\mathrm{PGL}_{2}(\mathbb{Z} / 5) \simeq \mathrm{S}_{5}$ on the projective line $\mathrm{P}^{1}(\mathbb{Z} / 5)$. The action of $\mathrm{GL}_{2}(\mathbb{Z} / 5)$ on the 12 -elements set $\left((\mathbb{Z} / 5)^{2}-\{0\}\right) /\{ \pm 1\}$ permutes the 6 disjoint pairs of the form $\{v, 2 v\}$, which defines a natural conjugacy class of embeddings

$$
\iota: \mathrm{GL}_{2}(\mathbb{Z} / 5) /\left\{ \pm \mathrm{I}_{2}\right\} \longrightarrow\{ \pm 1\}^{6} \rtimes \mathrm{S}_{5} .
$$

The group $\iota\left(\mathrm{GL}_{2}(\mathbb{Z} / 5) /\left\{ \pm \mathrm{I}_{2}\right\}\right)$ belongs to the second class of Proposition $2.10\left(\right.$ recall $\left.\operatorname{PSL}_{2}(\mathbb{Z} / 5) \simeq \mathrm{Alt}_{5}\right)$. The map $\iota$ is explicit enough to allow the computation of the conjugacy classes of the elements of 
$\iota\left(\mathrm{GL}_{2}(\mathbb{Z} / 5) /\left\{ \pm \mathrm{I}_{2}\right\}\right)$ viewed as a subgroup of $\mathrm{H}_{6} \supset\{ \pm 1\}^{6} \rtimes \mathrm{S}_{5}$ : they are gathered in Table 2.2 .

TABLE 2.2. The $\mathrm{H}_{6}$-conjugacy classes of the elements of $\mathrm{GL}_{2}(\mathbb{Z} / 5) /\left\{ \pm \mathrm{I}_{2}\right\}$.

\begin{tabular}{c|c|c|c|c|c|c|c|c|c|c|c} 
type & $1^{6}$ & $1^{6}$ & $1^{2} 2^{2}$ & $1^{2} 2^{2}$ & $2^{3}$ & $3^{2}$ & $3^{2}$ & 114 & 15 & 15 & 6 \\
\hline size & $1 / 240$ & $1 / 240$ & $1 / 16$ & $1 / 16$ & $1 / 12$ & $1 / 12$ & $1 / 12$ & $1 / 4$ & $1 / 10$ & $1 / 10$ & $1 / 6$
\end{tabular}

\section{Characteristic masses of root lattices}

3.1. Root systems and root lattices. Let $V$ be an Euclidean space. By a root of $V$ we mean an element $\alpha \in V$ with $\alpha \cdot \alpha=2$; we denote by $\mathrm{R}(V)$ the set of roots of $V$ (a sphere). For each $\alpha \in \mathrm{R}(V)$, the orthogonal reflection about $\alpha$ is an element $\mathrm{s}_{\alpha}$ of $\mathrm{O}(V)$, given by the formula $\mathrm{s}_{\alpha}(x)=x-(\alpha \cdot x) \alpha$.

An ADE root system in $V$ is a finite set $R \subset \mathrm{R}(V)$ generating $V$ as a real vector space, and such that for all $\alpha, \beta \in R$ we have $\alpha \cdot \beta \in \mathbb{Z}$ and $\mathrm{s}_{\alpha}(\beta) \in R$. In particular, $R$ is a root system in the sense of Bourbaki [4], and each irreducible component of $R$ is of type $\mathbf{A}_{n}$ with $n \geq 1, \mathbf{D}_{n}$ with $n \geq 4$, or $\mathbf{E}_{n}$ with $n=6,7,8$. The root lattice of $R$ is the lattice $\mathrm{Q}(R) \stackrel{\text { det }}{=} \sum_{\alpha \in R} \mathbb{Z} \alpha \subset V$ generated by $R$. This is an even lattice, and we have the important equality

$$
\mathrm{R}(V) \cap \mathrm{Q}(R)=R .
$$

If $L \subset V$ is any integral lattice, we denote by $\mathrm{R}(L)=L \cap \mathrm{R}(V)$ the set of roots of $L$. It follows at once from the definitions that $\mathrm{R}(L)$ is an ADE root system in the Euclidean subspace $U$ of $V$ generated by $\mathrm{R}(L)$. We say that $L$ is a root lattice if $\mathrm{R}(L)$ generates $L$ as an abelian group, i.e. if we have $L=\mathrm{Q}(\mathrm{R}(L))$ (hence $U=V)$. By definitions and (3.1), the map $R \mapsto \mathrm{Q}(R)$ is a bijection between the set of ADE root systems of $V$ and the set of root lattices of $V$, whose inverse is $L \mapsto \mathrm{R}(L)$.

We shall always use a bold font to denote an isomorphism class of root systems, and reserve the normal font for a root lattice with the corresponding root system. For instance, if for $n \geq 2$ we set $\mathrm{D}_{n}=\left\{\left(x_{i}\right) \in \mathbb{Z}^{n}, \sum_{i} x_{i} \equiv\right.$ $0 \bmod 2\}$ as in the introduction, then $\mathrm{R}\left(\mathrm{D}_{n}\right)$ is a root system of type $\mathbf{D}_{n}$ in the standard Euclidean space $\mathbb{R}^{n}$. We have also defined loc. cit. the lattice $\mathrm{E}_{n}$ for $n \equiv 0 \bmod 8$. It is easy to check $\mathrm{R}\left(\mathrm{E}_{n}\right)=\mathrm{R}\left(\mathrm{D}_{n}\right)$ for $n>8$ and that $\mathrm{R}\left(\mathrm{E}_{8}\right)$ is of type $\mathbf{E}_{8}$. We choose in an arbitrary way root lattices $\mathrm{A}_{n}$ for $n \geq 1$, as well as $\mathrm{E}_{6}$ and $\mathrm{E}_{7}$, whose root systems are of type $\mathbf{A}_{n}, \mathbf{E}_{6}$ and $\mathbf{E}_{7}$.

Let $L$ be an integral lattice in $V$ and set $R=\mathrm{R}(L)$. The $\mathrm{s}_{\alpha}$ with $\alpha$ in $R$ generate a subgroup of $\mathrm{O}(L)$ called the Weyl group of $L$, and denoted $\mathrm{W}(L)$. This is a normal subgroup of $\mathrm{O}(L)$, and we denote by $\mathrm{G}(L)=\mathrm{O}(L) / \mathrm{W}(L)$ the quotient group. Assume first that $L$ is the root lattice $\mathrm{Q}(R)$; in this 
case we also set $\mathrm{W}(R):=\mathrm{W}(L), \mathrm{O}(R):=\mathrm{O}(L)$ (this latter group is also denoted $\mathrm{A}(R)$ by Bourbaki) and $\mathrm{G}(R)=\mathrm{G}(L)$. As is well-known, $\mathrm{G}(R)$ is isomorphic to the automorphism group of the Dynkin diagram of $R$, and we have

$$
\mathrm{G}(R) \simeq \begin{cases}1 & \text { for } R \simeq \mathbf{A}_{1}, \mathbf{E}_{7}, \mathbf{E}_{8} \\ \mathrm{~S}_{3} & \text { for } R \simeq \mathbf{D}_{4} \\ \mathbb{Z} / 2 & \text { otherwise }\end{cases}
$$

Moreover, $\mathrm{W}(R)$ permutes the positive root systems ${ }^{10} R^{+}$of $R$, or equivalently the Weyl vectors ${ }^{11}$ of $R$, in a simply transitive way. Let us now go back to the case of an arbitrary $L$. The set $R$ is a root system in the Euclidean space $U$ generated by $R$, and the restriction $\sigma \mapsto \sigma_{\mid U}$ induces a morphism $\mathrm{O}(L) \rightarrow \mathrm{O}(R)$ and an isomorphism $\mathrm{W}(L) \stackrel{\sim}{\rightarrow} \mathrm{W}(R)$. It follows that $\mathrm{O}(L)$ permutes the Weyl vectors of $R$, and that $\mathrm{W}(L)$ permutes them simply transitively. So for any Weyl vector $\rho$ of $R$, the stabilizer $\mathrm{O}(L)_{\rho}$ of $\rho$ in $\mathrm{O}(L)$ is naturally isomorphic to $\mathrm{G}(L)$ and we have

$$
\mathrm{W}(L) \cap \mathrm{O}(L)_{\rho}=1, \quad \mathrm{O}(L)=\mathrm{W}(L) \cdot \mathrm{O}(L)_{\rho} \quad \text { and } \quad \mathrm{W}(L) \simeq \mathrm{W}(R),
$$

so that $\mathrm{O}(L)$ is the semi-direct product of $\mathrm{O}(L)_{\rho}$ by $\mathrm{W}(L)$.

3.2. Characteristic masses of irreducible root lattices. In this paragraph, we let $R \subset V$ be an $\mathbf{A D E}$ root system and $L=\mathrm{Q}(R)$. Set $n=\operatorname{dim} V$. Our aim is to determine the characteristic masses of $\mathrm{O}(L)$ and, more generally, the map $\mathrm{m}_{S}: \operatorname{Car}_{n} \rightarrow \mathbb{Q}_{\geq 0}$ where $S$ is any subset of the form $\sigma \mathrm{W}(L)$ with $\sigma \in \mathrm{O}(L)$ (see Section 1.2(ii) for the definition of $\mathrm{m}_{S}$ ). We assume first $R$ irreducible, and argue case by case.

(A) Case $\boldsymbol{R} \simeq \mathbf{A}_{\boldsymbol{n}}$ with $\boldsymbol{n} \geq \mathbf{1}$. We may assume that $V$ is the hyperplane of sum 0 vectors in $\mathbb{R}^{n+1}$ and $R=\left\{ \pm\left(\epsilon_{i}-\epsilon_{j}\right), 1 \leq i<j \leq n+1\right\}$, where $\epsilon_{1}, \ldots, \epsilon_{n+1}$ denotes the canonical basis of $\mathbb{R}^{n+1}$, and $L=\mathrm{A}_{n}$. The group $\mathrm{W}\left(\mathrm{A}_{n}\right)$ may be identified with the symmetric group $\mathrm{S}_{n+1}$, acting on $V$ by permuting coordinates.

Let $\mathcal{S}$ denote the set of integer sequences $\underline{m}=\left(m_{i}\right)_{i \geq 1}$ with $m_{i} \geq 0$ for each $i$, and $m_{i}=0$ for $i$ big enough. Let $\mathcal{A}_{n} \subset \mathcal{S}$ denote the subset of $\underline{m}$ such that $\sum_{i} i m_{i}=n+1$. For any $\underline{m}$ in $\mathcal{A}_{n}$, the elements of $\mathrm{S}_{n+1}$ whose cycle decomposition contains $m_{i}$ cycles of length $i$ for each $i$ form a single conjugacy class $\mathrm{C}_{\underline{m}} \subset \mathrm{S}_{n+1}$. We have furthermore $\left|\mathrm{C}_{\underline{m}}\right|=(n+1) ! / \mathrm{n}_{\underline{m}}$ with

$$
\mathrm{n}_{\underline{m}}=\prod_{i} m_{i} ! i^{m_{i}}
$$

\footnotetext{
${ }^{10}$ Recall that a positive root system in $R$ is a subset of the form $\{\alpha \in R, \varphi(\alpha)>0\}$ where $\varphi: V \rightarrow \mathbb{R}$ is a linear form with $0 \notin \varphi(R)$.

${ }^{11}$ A Weyl vector of $R$ is a vector of the form $\rho=\frac{1}{2} \sum_{\alpha \in R^{+}} \alpha$ for $R^{+}$a positive root system of $R$. In particular we have $2 \rho \in \mathrm{Q}(R)$.
} 
The characteristic polynomial of $\mathrm{C}_{\underline{m}}$ acting on $V$ is

$$
\mathrm{P}_{\underline{m}}=(t-1)^{-1} \prod_{i}\left(t^{i}-1\right)^{m_{i}},
$$

since $\mathbb{R}^{n+1} / V$ is the trivial representation of $\mathrm{S}_{n+1}$. The following trivial lemma even shows that we have $\mathrm{P}_{\underline{m}} \neq \mathrm{P}_{{\underline{m^{\prime}}}^{\prime}}$ for $\underline{m} \neq \underline{m^{\prime}}$.

Lemma 3.3. The polynomials $t^{l}-1$, with $l \geq 1$, are $\mathbb{Z}$-linearly independent in the multiplicative group of the field $\mathbb{Q}(t)$.

As a consequence, we obtain the:

Corollary 3.4. For $n \geq 1$, we have $\mathrm{m}_{\mathrm{W}\left(\mathrm{A}_{n}\right)}\left(\mathrm{P}_{\underline{m}}\right)=1 / \mathrm{n}_{\underline{m}}$ for $\underline{m}$ in $\mathcal{A}_{n}$, and $\mathrm{m}_{\mathrm{W}\left(\mathrm{A}_{n}\right)}(P)=0$ for all other $P$ in $\operatorname{Car}_{n}$.

As is easily seen, the element $-1=-\mathrm{id}_{V}$ is in $\mathrm{W}\left(\mathrm{A}_{n}\right)$ if and only if $n=1$, and we have $\mathrm{O}\left(\mathrm{A}_{n}\right)=\mathrm{W}\left(\mathrm{A}_{n}\right) \cup-\mathrm{W}\left(\mathrm{A}_{n}\right)$ (this fits of course Formula (3.2)). The map $\mathrm{m}_{S}$ for the coset $S=-\mathrm{W}\left(\mathrm{A}_{n}\right)$ is deduced from $\mathrm{m}_{\mathrm{W}\left(\mathrm{A}_{n}\right)}$ by the following trivial lemma:

Lemma 3.5. Let $S$ be a finite subset of $\mathrm{O}(V)$ with $\operatorname{dim} V=n$. Then for all $P \in \mathbb{R}[t]$ we have $\mathrm{m}_{-S}(P)=\mathrm{m}_{S}(Q)$ with $Q(t)=(-1)^{n} P(-t)$.

(D) Case $\boldsymbol{R} \simeq \mathbf{D}_{\boldsymbol{n}}$ with $\boldsymbol{n} \geq \mathbf{3}$. We may assume $V=\mathbb{R}^{n}, R=\left\{ \pm \epsilon_{i} \pm\right.$ $\left.\epsilon_{j}, 1 \leq i<j \leq n\right\}$ where $\epsilon_{1}, \ldots, \epsilon_{n}$ denote again the canonical basis of $V$, and $L=\mathrm{D}_{n}$. The lattice $\mathrm{D}_{n}$ is the largest even sublattice of the standard lattice

$$
\mathrm{I}_{n}=\mathbb{Z}^{n}=\oplus_{i=1}^{n} \mathbb{Z} \epsilon_{i},
$$

and thus $\mathrm{O}\left(\mathrm{I}_{n}\right)$ is a subgroup of $\mathrm{O}\left(\mathrm{D}_{n}\right)$. This group $\mathrm{O}\left(\mathrm{I}_{n}\right)$ is nothing else than the hyperoctahedral group $\mathrm{H}_{n}$ already introduced in Section 2.1: we have

$$
\mathrm{O}\left(\mathrm{I}_{n}\right)=\mathrm{H}_{n}=\{ \pm 1\}^{n} \rtimes \mathrm{S}_{n}
$$

where $\mathrm{S}_{n}$ (resp. $\{ \pm 1\}^{n}$ ) acts on $\mathbb{R}^{n}$ by permuting coordinates (resp. sign changes). As is well-known, $\mathrm{W}\left(\mathrm{D}_{n}\right)$ is the index 2 subgroup ker s of $\mathrm{O}\left(\mathrm{I}_{n}\right)$ (recall $\mathrm{s}$ is defined by Formula (2.1)). By (3.2) we also have

$$
\mathrm{O}\left(\mathrm{I}_{n}\right)=\mathrm{O}\left(\mathrm{D}_{n}\right) \text { for } n \neq 4 \text { and } \mathrm{G}\left(\mathrm{D}_{4}\right) \simeq \mathrm{S}_{3} \text { (triality). }
$$

The conjugacy classes of $\mathrm{H}_{n}$ have been recalled in Section 2.3. Let $\mathcal{D}_{n} \subset$ $\mathcal{S} \times \mathcal{S}$ be the subset of $\left(\underline{m}^{+}, \underline{m}^{-}\right)$with $\sum_{i} i\left(m_{i}^{+}+m_{i}^{-}\right)=n$. For any $\left(\underline{m}^{+}, \underline{m}^{-}\right)$in $\mathcal{D}_{n}$ the elements of $\mathrm{H}_{n}$ whose cycle decomposition contains $m_{i}^{+}$ (resp. $m_{i}^{-}$) cycles of length $i$ with sign +1 (resp. -1 ) for each $i$ form a single conjugacy class $\mathrm{C}_{\underline{m}^{+}, \underline{m}^{-}} \subset \mathrm{H}_{n}$. We easily check $\left|\mathrm{C}_{\underline{m}^{+}, \underline{m}^{-}}\right|=2^{n} n ! / \mathrm{n}_{\underline{m}^{+}, \underline{m}^{-}}$ with

$$
\mathrm{n}_{\underline{m}^{+}, \underline{m}^{-}}=\prod_{i} m_{i}^{+} ! m_{i}^{-} !(2 i)^{m_{i}^{+}+m_{i}^{-}}
$$


and $\mathrm{s}\left(\mathrm{C}_{\underline{m}^{+}, \underline{m}^{-}}\right)=(-1)\left|\underline{m}^{-}\right|$where we have set $|\underline{m}|=\sum_{i} m_{i}$ for $\underline{m} \in \mathcal{S}$. The characteristic polynomial of $\mathrm{C}_{\underline{m}^{+}, \underline{m}^{-}}$acting on $V$ is

$$
\mathrm{P}_{\underline{m}^{+}, \underline{m}^{-}}=\prod_{i}\left(t^{i}-1\right)^{m_{i}^{+}}\left(t^{i}+1\right)^{m_{i}^{-}}=\prod_{i}\left(t^{i}-1\right)^{m_{i}^{+}-m_{i}^{-}+m_{i / 2}^{-}},
$$

where we have set $m_{i / 2}^{-}=0$ for $i$ odd, and used for $i \geq 1$ the relation $\left(t^{i}-1\right)\left(t^{i}+1\right)=\left(t^{2 i}-1\right)$. In contrast with the $\mathbf{A}_{n}$ case, we may thus have $\mathrm{P}_{\underline{m}^{+}, \underline{m}^{-}}=\mathrm{P}_{\underline{n}^{+}, \underline{n}^{-}}$for distinct $\left(\underline{m}^{+}, \underline{m}^{-}\right)$and $\left(\underline{n}^{+}, \underline{n}^{-}\right)$in $\mathcal{D}_{n}$. This leads us to introduce the subset

$$
\mathcal{D}_{n}^{\prime}=\left\{\left(\underline{m}^{+}, \underline{m}^{-}\right) \in \mathcal{D}_{n} \mid m_{i}^{+} m_{i}^{-}=0 \text { for all } i \geq 1\right\} .
$$

Lemma 3.3 shows that we have $\mathrm{P}_{\underline{m}^{+}, \underline{m}^{-}} \neq \mathrm{P}_{\underline{n}^{+}, \underline{n}^{-}}$for $\left(\underline{m}^{+}, \underline{m}^{-}\right) \neq\left(\underline{n}^{+}, \underline{n}^{-}\right)$ in $\mathcal{D}_{n}^{\prime}$. We reduce to $\mathcal{D}_{n}^{\prime}$ as follows. Consider the following map $\phi: \mathcal{D}_{n} \rightarrow \mathcal{D}_{n}$ :

(i) if $\left(\underline{m}^{+}, \underline{m}^{-}\right) \in \mathcal{D}_{n}^{\prime}$ set $\phi\left(\underline{m}^{+}, \underline{m}^{-}\right)=\left(\underline{m}^{+}, \underline{m}^{-}\right)$,

(ii) otherwise there is a smallest $j \geq 1$ with $m_{j}^{+} m_{j}^{-} \neq 0$ and we set $\phi\left(\underline{m}^{+}, \underline{m}^{-}\right)=\left(\underline{n}^{+}, \underline{n}^{-}\right)$with $\left(n_{i}^{+}, n_{i}^{-}\right)=\left(m_{i}^{+}, m_{i}^{-}\right)$for $i \neq j$ or $i \neq 2 j$, and with $\left(n_{j}^{+}, n_{j}^{-}\right)=\left(m_{j}^{+}-1, n_{j}^{+}-1\right)$ and $\left(n_{2 j}^{+}, n_{2 j}^{-}\right)=$ $\left(m_{2 j}^{+}+1, m_{2 j}^{-}\right)$.

It is clear that we have $\mathrm{P}_{\phi\left(\underline{m}^{+}, \underline{m}^{-}\right)}=\mathrm{P}_{\underline{m}^{+}, \underline{m}^{-}}$for all $\left(\underline{m}^{+}, \underline{m}^{-}\right)$in $\mathcal{D}_{n}$, and that for each $m=\left(\underline{m}^{+}, \underline{m}^{-}\right) \in \mathcal{D}_{n}$ the sequence $m, \phi(m), \phi^{2}(m), \ldots$ is eventually constant and equal to some element of $\mathcal{D}_{n}^{\prime}$, that we denote by $\psi(m)$.

Corollary 3.6. Let $\sigma \in \mathrm{O}\left(\mathrm{I}_{n}\right)$. For all $\left(\underline{m}^{+}, \underline{m}^{-}\right)$in $\mathcal{D}_{n}^{\prime}$ we have

$$
\mathrm{m}_{\sigma \mathrm{W}\left(\mathrm{D}_{n}\right)}\left(\mathrm{P}_{\underline{m}^{+}, \underline{m}^{-}}\right)=\sum \frac{1}{\mathrm{n}_{\underline{n}^{+}, \underline{n}^{-}}}
$$

the sum being over all the $\left(\underline{n}^{+}, \underline{n}^{-}\right)$in $\mathcal{D}_{n}$ with $\psi\left(\underline{n}^{+}, \underline{n}^{-}\right)=\left(\underline{m}^{+}, \underline{m}^{-}\right)$and $(-1)^{\left|\underline{m}^{-}\right|}=\mathrm{s}(\sigma)$. We have $\mathrm{m}_{\sigma \mathrm{W}\left(\mathrm{D}_{n}\right)}(P)=0$ for all other $P$ in $\operatorname{Car}_{n}$.

We have $G\left(D_{4}\right) \simeq S_{3}$ so it remains to determine $m_{\sigma W\left(D_{4}\right)}$ for the 6 possible classes $\sigma \mathrm{W}\left(\mathrm{D}_{4}\right)$. A first general reduction is the following lemma:

Lemma 3.7. Let $L$ be an integral lattice, as well as elements $\sigma_{1}, \sigma_{2}$ in $\mathrm{O}(L)$ whose images in $\mathrm{G}(L)$ are conjugate. Then we have $\mathrm{m}_{\sigma_{1} \mathrm{~W}(L)}=\mathrm{m}_{\sigma_{2} \mathrm{~W}(L)}$.

Proof. Write $\sigma_{2}=\gamma \sigma_{1} \gamma^{-1} w_{0}$ with $\gamma$ in $\mathrm{O}(L)$ and $w_{0}$ in $\mathrm{W}(L)$. For $w \in$ $\mathrm{W}(L)$ we have $\operatorname{det}\left(t-\sigma_{2} w\right)=\operatorname{det}\left(t-\sigma_{1} \gamma^{-1} w_{0} w \gamma\right)$. We conclude as $w \mapsto$ $\gamma^{-1} w_{0} w \gamma$ is a bijection of the normal subgroup $\mathrm{W}(L)$ of $\mathrm{O}(L)$.

In particular, $\mathrm{m}_{\sigma \mathrm{W}\left(\mathrm{D}_{4}\right)}$ is already given by Lemma 3.6 whenever the image of $\sigma$ in $\mathrm{G}\left(\mathrm{D}_{4}\right) \simeq \mathrm{S}_{3}$ has order 1 or 2 , and does not depend on $\sigma$ if this image 
has order 3 . There are many ways to determine $\mathrm{m}_{\sigma \mathrm{W}\left(\mathrm{D}_{4}\right)}$ in this latter case. One way is to consider first the set

$$
R^{\prime}=\left\{v \in \mathrm{D}_{4}, v \cdot v=4\right\}=\left\{ \pm 2 \epsilon_{i} \mid i=1, \ldots, 4\right\} \cup\left\{\sum_{i=1}^{4} \pm \epsilon_{i}\right\}
$$

and observe that we have $\alpha \cdot x \in 2 \mathbb{Z}$ for all $\alpha \in R^{\prime}$ and $x \in \mathrm{D}_{4}$. In particular, $\frac{1}{\sqrt{2}} R^{\prime}$ is a root system (of type $\left.\mathbf{D}_{4}\right)$ in $\mathbb{R}^{4}$ and we have $\mathrm{W}\left(\frac{1}{\sqrt{2}} R^{\prime}\right) \subset \mathrm{O}\left(\mathrm{D}_{4}\right)$. The two roots $\alpha=\sqrt{2} \epsilon_{1}$ and $\beta=\frac{1}{\sqrt{2}}\left(\epsilon_{1}+\epsilon_{2}+\epsilon_{3}+\epsilon_{4}\right)$ are in $\frac{1}{\sqrt{2}} R^{\prime}$ with $\alpha \cdot \beta=1$, and the order 3 element

$$
\sigma_{0}:=\mathrm{s}_{\beta} \circ \mathrm{s}_{\alpha}=\frac{1}{2}\left(\begin{array}{cccc}
-1 & -1 & -1 & -1 \\
1 & 1 & -1 & -1 \\
1 & -1 & 1 & -1 \\
1 & -1 & -1 & 1
\end{array}\right)
$$

of $\mathrm{O}\left(\mathrm{D}_{4}\right)$ does not belong to $\mathrm{W}\left(\mathrm{D}_{4}\right)$. It is trivial to enumerate with a computer the $2^{3} 4 !=384$ elements of $\sigma_{0} \mathrm{~W}\left(\mathrm{D}_{4}\right)$ and to list their characteristic polynomials. We obtain:

Corollary 3.8. Let $\sigma$ be an element of order 3 in $\mathrm{G}\left(\mathrm{D}_{4}\right)$. The $\mathrm{m}_{\sigma \mathrm{W}\left(\mathrm{D}_{4}\right)}(P)$ with $P \in \mathrm{Car}_{4}$ are given by Table C.1.

In fact, the reasoning above can be pushed a little further: it turns out that $R^{\prime \prime}=R \coprod R^{\prime}$ is a root system of type $\mathbf{F}_{4}$ in $\mathbb{R}^{4}$ (not ADE of course) and that we have $\mathrm{W}\left(R^{\prime \prime}\right)=\left\langle\mathrm{W}(R), \mathrm{W}\left(\frac{1}{\sqrt{2}} R^{\prime}\right)\right\rangle=\mathrm{O}\left(\mathrm{D}_{4}\right)$. But the conjugacy classes of $\mathrm{W}(S)$, with $S$ any irreducible root system of exceptional type, have been listed and studied in a conceptual way by Carter in [5], including their characteristic polynomials (see [5, p. $22 \& 23]$ ). The map $\mathrm{m}_{\mathrm{O}_{\left(\mathrm{D}_{4}\right)} \text { may }}$ be deduced in particular from Table 8 of [5]. The map $\mathrm{m}_{\sigma_{0} \mathrm{~W}\left(\mathrm{D}_{4}\right)}$ follows then from the equality $\mathrm{m}_{\mathrm{O}\left(\mathrm{D}_{4}\right)}=-\frac{1}{3} \mathrm{~m}_{\mathrm{W}\left(\mathrm{D}_{4}\right)}+\frac{1}{2} \mathrm{~m}_{\mathrm{O}\left(\mathrm{I}_{4}\right)}+\frac{1}{3} \mathrm{~m}_{\sigma_{0} \mathrm{~W}\left(\mathrm{D}_{4}\right)}$.

Remark 3.9. Assume $R$ is an irreducible root system. It follows from (3.2) that two elements of $\mathrm{G}(R)$ are conjugate if and only if they have the same order, which is always 1,2 or 3. In particular, Lemma 3.7 shows that for $\sigma$ in $\mathrm{O}(R)$ the map $\mathrm{m}_{\sigma \mathrm{W}(R)}$ only depends on the order of $\sigma$ in $\mathrm{G}(R)$.

(E) Cases $R \simeq \mathbf{E}_{n}$ with $\boldsymbol{n}=\mathbf{6 , 7}$ and 8. The aforementioned results of Carter also allow to deduce $\mathrm{m}_{\mathrm{W}\left(\mathrm{E}_{n}\right)}$ for $n=6,7$ and 8 (using Tables 9, 10 and 11 loc. cit). Alternatively, and as a useful check, these masses can also be computed directly using a variant of the Algorithm A explained in Section 1.4. Indeed, choosing a positive system $R^{+} \subset R$, we may view $\mathrm{W}(R)$ as the subgroup of $\mathrm{O}(V)$ generated by the $n$ reflections $\mathrm{s}_{\alpha}$, with $\alpha$ a simple root in $R^{+}$. As $\mathrm{W}(R)$ acts faithfully and transitively on $R$, it is also the subgroup of the permutation group of $R$ generated by these $n$ permutations $\mathrm{s}_{\alpha}$, with $|R|=72$ (case $\left.n=6\right),|R|=126$ (case $n=7$ ) or $|R|=240$ (case 
$n=8)$. Applying GAP's ConjugacyClasses algorithm to this permutation group, we obtain representatives and cardinalities of the conjugacy classes of $\mathrm{W}(R)$, and it only remains to compute their characteristic polynomials. All in all, these computations only take a few seconds for the computer. Both methods lead to the:

Corollary 3.10. For $n=6,7$ and 8 , the $\mathrm{m}_{\mathrm{W}\left(\mathrm{E}_{n}\right)}(P)$ with $P \in \mathrm{Car}_{n}$ are given by Tables C.2, C.3 and C.4.

Note that for $n=7,8$ we have $\mathrm{O}\left(\mathrm{E}_{n}\right)=\mathrm{W}\left(\mathrm{E}_{n}\right)$ (no non trivial diagram automorphism). For $n=6$, we have $\mathrm{O}\left(\mathrm{E}_{6}\right)=\mathrm{W}\left(\mathrm{E}_{6}\right) \amalg-\mathrm{W}\left(\mathrm{E}_{6}\right)$, but the map $\mathrm{m}_{-\mathrm{W}\left(\mathrm{E}_{6}\right)}$ is deduced from $\mathrm{m}_{\mathrm{W}\left(\mathrm{E}_{6}\right)}$ using Lemma 3.5.

3.11. The non irreducible case. Assume now $R$ is a non necessarily irreducible ADE root system in $V$, set $L=\mathrm{Q}(R)$ and fix $\sigma$ in $\mathrm{O}(R)$. Our aim is to give a formula for $\mathrm{m}_{\sigma \mathrm{W}(R)}$. Write $R$ as the disjoint union of its irreducible components $R=\coprod_{i \in I} R_{i}$. We have

$$
L=\bigoplus_{i \in I}^{\perp} \mathrm{Q}\left(R_{i}\right) \text { and } \mathrm{W}(R)=\prod_{i \in I} \mathrm{~W}\left(R_{i}\right) .
$$

The element $\sigma$ induces a permutation of the set $\left\{R_{i} \mid i \in I\right\}$ of irreducible components of $R$. We write $\sigma=c_{1} c_{2} \cdots c_{r}$ the cycle decomposition of this permutation. For each $j=1, \ldots, r$, we choose an irreducible component $S_{j}$ of $R$ in the support of $c_{j}$, denote by $s_{j}=\operatorname{dim} \mathrm{Q}\left(S_{j}\right)$ the rank of $S_{j}$ and by $l_{j}$ the length of the cycle $c_{j}$. For each $j$ we have $\sigma^{l_{j}}\left(S_{j}\right)=S_{j}$ and we denote by $\tau_{j}$ the restriction of $\sigma^{l_{j}}$ to $\mathrm{Q}\left(S_{j}\right)$; so $\tau_{j}$ is an element of $\mathrm{O}\left(S_{j}\right)$.

Proposition 3.12. In the setting above, we have for all $P$ in $\mathrm{Car}_{n}$

$$
\mathrm{m}_{\sigma \mathrm{W}(R)}(P)=\sum_{\left(P_{1}, \ldots, P_{r}\right)} \prod_{j=1}^{r} \mathrm{~m}_{\tau_{j} \mathrm{~W}\left(S_{j}\right)}\left(P_{j}\right)
$$

summing over all $\left(P_{1}, \ldots, P_{r}\right) \in \operatorname{Car}_{s_{1}} \times \cdots \times \operatorname{Car}_{s_{r}}$ with $\prod_{j=1}^{r} P_{j}\left(t^{l_{j}}\right)=P(t)$.

The first ingredient in the proof is the following trivial lemma.

Lemma 3.13. For $i=1,2$, let $V_{i}$ be an Euclidean space and $\Gamma_{i} \subset \mathrm{O}\left(V_{i}\right)$ a finite subset. Set $V=V_{1} \perp V_{2}$ and view $\Gamma=\Gamma_{1} \times \Gamma_{2}$ as a subset of $\mathrm{O}(V)$. For all monic polynomials $P$ in $\mathbb{R}[t]$ of degree $\operatorname{dim} V$ we have

$$
\mathrm{m}_{\Gamma}(P)=\sum_{\left(P_{1}, P_{2}\right)} \mathrm{m}_{\Gamma_{1}}\left(P_{1}\right) \mathrm{m}_{\Gamma_{2}}\left(P_{2}\right)
$$

the sum being over the $\left(P_{1}, P_{2}\right)$, with $P_{i} \in \mathbb{R}[t]$ monic of degree $\operatorname{dim} V_{i}$, and with $P_{1} P_{2}=P$. 
Proof of Proposition 3.12. Applying Lemma 3.13, we may and do assume $r=1$, i.e. that $\sigma$ permutes transitively the irreducible components of $R$. In this case, we simply write $(S, s, l, \tau)$ instead of $\left(S_{1}, s_{1}, l_{1}, \tau_{1}\right)$. We may also assume that we have $I=\{0, \ldots, l-1\}$ and $R_{i}=\sigma^{i}(S)$ for $0 \leq i<l$. In particular, we have $\mathrm{W}(R)=\prod_{0 \leq i<l} \sigma^{i} \mathrm{~W}(S) \sigma^{-i}$. Choose a $\mathbb{Z}$-basis $e=\left(e_{1}, \ldots, e_{s}\right)$ of $\mathrm{Q}(S)$ and consider the following $\mathbb{Z}$-basis of $L$ :

$$
f=\left(e_{1}, \ldots, e_{s}, \sigma\left(e_{1}\right), \ldots, \sigma\left(e_{s}\right), \ldots, \sigma^{l-1}\left(e_{1}\right), \ldots, \sigma^{l-1}\left(e_{s}\right)\right) .
$$

For all $w=\left(\sigma^{i} w_{i} \sigma^{-i}\right)_{0 \leq i<l}$ in $\mathrm{W}(R)$, the matrix of $\sigma w$ in the basis $f$ is

$$
\left(\begin{array}{ccccc} 
& & & & M W_{l-1} \\
W_{0} & & & & \\
& W_{1} & & \\
& & \ddots & \\
& & & W_{l-2} & \\
& & &
\end{array}\right)
$$

where $W_{i}$ is the matrix of $w_{i} \in \mathrm{W}(S)$ in the basis $e$, and $M$ is the matrix of $\tau=\sigma^{l}$ in the basis $e$. By Lemma 3.14 below, it follows that the multiset of polynomials $\operatorname{det}(t-\sigma w)$ (counted with their multiplicities) when $w$ varies in $\mathrm{W}(R)$, coincides with that of polynomials $\operatorname{det}\left(t^{s}-\tau w_{l-1} w_{l-2} \cdots w_{0}\right)$ when the $l$-tuple $\left(w_{0}, w_{1}, \ldots, w_{l-1}\right)$ varies in $\mathrm{W}(S)^{l}$. As $\mathrm{W}(S)$ is a group, this multiset is also $|\mathrm{W}(S)|^{l-1}$ times the multiset of the $\operatorname{det}\left(t^{s}-\tau w\right)$ when $w$ varies in $\mathrm{W}(S)$, and we are done.

Lemma 3.14. For any $W_{0}, W_{1}, \ldots, W_{l-1}$ and $M$ in $\mathrm{M}_{s}(\mathbb{C})$, the characteristic polynomial of the matrix (3.5) of size sl is $\operatorname{det}\left(t^{s}-M W_{l-1} W_{l-2} \cdots W_{0}\right)$.

Proof. By continuity, we may assume $W_{i} \in \mathrm{GL}_{s}(\mathbb{C})$ for each $i$. Up to conjugating $(3.5)$ by the diagonal matrix $\left(1, W_{0}, W_{1} W_{0}, \ldots, W_{l-2} \cdots W_{1} W_{0}\right)$ we may assume $W_{0}=W_{1}=\cdots=W_{l-1}=\mathrm{id}_{s}$. But in this case, the entries of (3.5) commute and we conclude by [31] and the following well-known fact (applied to $Q=t^{s}-a$ ): the characteristic polynomial of the companion matrix of a given monic polynomial $Q$ is the polynomial $Q$ itself.

\section{An algorithm computing characteristic masses}

4.1. Algorithm B. Consider the following algorithm, which takes as input an integral lattice $L$ in the standard Euclidean space $V=\mathbb{R}^{n}$ :

B1. Compute the root system $R=\mathrm{R}(L)$, a positive root system $R^{+} \subset R$ and the associate Weyl vector $\rho=\frac{1}{2} \sum_{\alpha \in R^{+}} \alpha$.

B2. Determine a set $\mathcal{G}$ of generators of the stabilizer $\mathrm{O}(L)_{\rho}$ of $\rho$ in $\mathrm{O}(L)$.

B3. Compute the set $\mathcal{S}=\mathrm{S}(L)$ defined on p. 551 and view $\mathrm{O}(L)_{\rho}$ as the subgroup of permutations of $\mathcal{S}$ generated by $\mathcal{G}$.

B4. Use permutation groups algorithms to determine the sizes $\left(m_{j}\right)_{j \in J}$ and representatives $\left(\gamma_{j}\right)_{j \in J}$ of the conjugacy classes $\left(c_{j}\right)_{j \in J}$ of $\mathrm{O}(L)_{\rho}$. 
B5. Compute the set $\operatorname{Irr}(R)$ of irreducible components of $R$, the isomorphism class of each such component, and a basis of the orthogonal $R^{\perp}$ of $R$ in $V$.

B6. For each $j$ in $J$, compute:

- the characteristic polynomial $P_{j}$ of $\gamma_{j}$ on $R^{\perp}$,

- a set of representatives $\operatorname{Irr}_{j} \subset \operatorname{Irr}(R)$ of the orbits for the action of $\gamma_{j}$ on $\operatorname{Irr}(R)$,

- for each $S \in \operatorname{Irr}_{j}$, the size $l_{S}$ of its $\gamma_{j}$-orbit and the order $d_{S} \in$ $\{1,2,3\}$ of the permutation $\gamma_{j}^{l_{S}}$ of $S$.

B7. For each $\left(S, d_{S}\right) \in \operatorname{Irr}(R) \times\{1,2,3\}$ found in B6, compute $\mathrm{m}_{\tau \mathrm{W}(S)}$ using the results of Section 3.2, where $\tau$ is any element of order $d_{S}$ in $\mathrm{O}(S) / \mathrm{W}(S)$ (see Remark 3.9).

B8. Using Proposition 3.12 and step B7, deduce for each $j$ in $J$ the map $\mathrm{m}_{\gamma_{j} \mathrm{~W}(R)}$.

B9. For each $j$ in $J$, define $M_{j}: \operatorname{Car}_{n} \rightarrow \mathbb{Q}$ by setting $M_{j}(P)=$ $\mathrm{m}_{\gamma_{j} \mathrm{~W}(R)}(Q)$ if we have $P=Q P_{j}$, and $M_{j}(P)=0$ otherwise.

B10. Return $\frac{\sum_{j \in J} m_{j} M_{j}}{\sum_{j \in J} m_{j}}$.

We will say more about each step of this algorithm in Section 4.3. Recall from (3.3) that we have a semi-direct product $\mathrm{O}(L)=\mathrm{W}(L) \rtimes \mathrm{O}_{\rho}(L)$ and that the restriction to the subspace $U=\mathrm{Q}(R) \otimes \mathbb{R}$ of $V$ induces a morphism res : $\mathrm{O}(L) \rightarrow \mathrm{O}(R)$, an isomorphism $\mathrm{W}(L) \stackrel{\sim}{\rightarrow} \mathrm{W}(R)$ and a morphism $\mathrm{O}(L)_{\rho} \rightarrow \mathrm{O}(R)_{\rho}$. Together with (3.2), this explains why the elements $d_{S}$ introduced in the step B6 are indeed in $\{1,2,3\}$. Moreover, the more correct notation for $\gamma_{j} \mathrm{~W}(R)$ in B8 should be res $\left(\gamma_{j}\right) \mathrm{W}(R)$. For $j$ in $J$, we have $M_{j}=\mathrm{m}_{\gamma_{j} \mathrm{~W}(L)}$ as $\mathrm{W}(L)$ acts trivially on $R^{\perp}$. Last but not least, Lemma 3.7 shows

We have proved the:

$$
\mathrm{m}_{\mathrm{O}(L)}=\frac{\sum_{j \in J} m_{j} \mathrm{~m}_{\gamma_{j} \mathrm{~W}(L)}}{\sum_{j \in J} m_{j}}
$$

\section{Proposition 4.2. Algorithm B returns $\mathrm{m}_{\mathrm{O}(L)}$.}

4.3. Precisions and an implementation. We now discuss more precisely the steps of Algorithm B, as well as some aspects of our implementation: see [6] for the source code and a documentation of the PARI/GP function masses_calc (requiring GAP) that we developped. Its input is a Gram matrix G of the lattice $L$, which is thus viewed as the lattice $\mathbb{Z}^{n}$ equipped with the inner product defined by G.

B1. Apply the Fincke-Pohst algorithm [23] to G to compute $R \subset \mathbb{Z}^{n}$. In PARI's implementation, qfminim(G) [3] returns a set $T \subset \mathbb{Z}^{n}$ with $T \cup-T=$ $R$ consisting of all the elements of $R$ lying in a certain half-space of $\mathbb{R}^{n}$ : this is a positive system, and we simply choose $R^{+}=T$. 
B2. Let $\mathrm{b}$ be the Gram matrix of the $\mathbb{Z}$-valued bilinear form $(x, y) \mapsto$ $4(\rho \cdot x)(\rho \cdot y)$ in the canonical basis of $\mathbb{Z}^{n}$. Apply the Plesken-Souvignier algorithm [40] to the pair of matrices $(G, b)$. This is implemented in PARI/GP as qfauto ( $[\mathrm{G}, \mathrm{b}]$ ) [2] (following Souvignier's $C$ code). It returns a set $\mathcal{G}^{\prime} \subset$ $\mathrm{GL}_{n}(\mathbb{Z})$ of generators of the subgroup of $\mathrm{O}(L)$ whose elements $g$ satisfy $g \rho= \pm \rho$. For each $g \in \mathcal{G}^{\prime}$, determine the sign $\epsilon_{g}$ with $g \rho=\epsilon_{g} \rho$. Define ${ }^{12} \mathcal{G}$ as the set of $\epsilon_{g} g$ with $g \in \mathcal{G}^{\prime}$.

B3. Apply recursively the Fincke-Pohst algorithm to find $\mathcal{S} \subset \mathbb{Z}^{n}$, as explained on p. 551. Choose arbitrarily an ordering $\psi: \mathcal{S} \stackrel{\sim}{\rightarrow}\{1, \ldots, N\}$. For each $g$ in $\mathcal{G}$, compute the permutation $\sigma_{g}=\psi \circ g \circ \psi^{-1}$ in the symmetric group $\mathrm{S}_{N}$. For later use, also extract a basis $\mathcal{S}_{0} \subset \mathcal{S}$ of $\mathbb{R}^{n}$.

B4. Apply GAP's ConjugacyClasses algorithm to the subgroup $H$ of $\mathrm{S}_{N}$ generated by the $\sigma_{g}$ with $g$ in $\mathcal{G}$. It returns a list of representatives $\left(r_{j}\right)_{j \in J}$ of the conjugacy classes of $H$, as well as their cardinalities $\left(m_{j}\right)_{j \in J}$. Each $r_{j}$ is a permutation of $\{1, \ldots, N\}$. Using the subset $\mathcal{S}_{0}$ introduced in B3, compute the matrix $\gamma_{j} \in \mathrm{GL}_{n}(\mathbb{Z})$ of the element of $\mathrm{O}(L)_{\rho}$ corresponding to $r_{j}$ under the natural isomorphism $H \simeq \mathrm{O}(L)_{\rho}$.

B5. Compute first the basis $B$ of the root system $R$ associated to $R^{+}$, using $B=\left\{\alpha \in R^{+} \mid \alpha \cdot \rho=1\right\}$. Define a graph with set of vertices $B$, and with an edge between $b, b^{\prime} \in B$ if and only if we have $b \cdot b^{\prime} \neq 0$. Determine the connected components $B=\coprod_{i \in I} B_{i}$ of this graph. For $i$ in $I$ define $R_{i}^{+}$as the subset of elements $\alpha$ in $R^{+}$with $\alpha \cdot B_{i} \neq 0$. We have $\operatorname{Irr}(R)=\left\{R_{i} \mid i \in I\right\}$. The isomorphism class of the ADE root system $R_{i}=R_{i}^{+} \cup-R_{i}^{+}$is uniquely determined by its rank $\left|B_{i}\right|$ and its cardinality $2\left|R_{i}^{+}\right|$.

B6. Use $i \mapsto R_{i}$ to identify $I$ with $\operatorname{Irr}(R)$. Compute the Weyl vector $\rho_{i}=$ $\frac{1}{2} \sum_{\alpha \in R_{i}^{+}} \alpha$ of $R_{i}$ for each $i$ in $I$. Fix $j \in J$. There is a unique permutation $\tau_{j}$ of $I$ such that $\gamma_{j}\left(\rho_{i}\right)=\rho_{\tau_{j}(i)}$ for all $i$ in $I$. Compute $\tau_{j}$ and determine its cycle decomposition.

Steps B7-B10 are theoretically straightforward. Nevertheless, the efficient implementation of these steps depends on the way the maps $\mathrm{m}_{S}$ are represented: see the documentation in [6] for more about the (imperfect) way we proceed in gp. In the end, masses_calc(G) returns the vector $[a, b, c, d]$ where:

- $a$ is the vector of all $[P, m]$ with $P$ in $\operatorname{Car}_{n}$ and $m=\mathrm{m}_{\mathrm{O}(L)}(P)$ with $m \neq 0$,

- $b$ is the isomorphism class of the root system $\mathrm{R}(L)$,

\footnotetext{
${ }^{12}$ An alternative (cleaner) method to compute the stabilizer in $\mathrm{O}(L)$ of a given element $x$ of $L$ would be to simply add the condition $v_{i} \cdot x=b_{i} \cdot x$ for all $i \leq k$ in the definition of a $k$-partial automorphism in $[40, \S 3]$, as well as a similar constraint in the definition of their fingerprint in $[40, \S 4]$. The main advantage of the trick we use is that we do not have to modify the code of the PARI port of Souvignier's program.
} 
- $c$ is the vector $\left(c_{j}\right)_{j \in J}$ where $c_{j}$ encodes both the cycle decomposition of $\gamma_{j}$ on $\operatorname{Irr}(R)$ and the integers $d_{S}$ for each $S$ in $\operatorname{Irr}_{j}$,

- $d$ is the vector $\left(d_{j}\right)_{j \in J}$ with $d_{j}=\left[P_{j}, m_{j} / M\right]$ and $M=\sum_{j \in J} m_{j}$.

\section{The characteristic masses of Niemeier Lattices with roots}

The aim of this section is to explain a way to determine the characteristic masses of the Niemeier lattices with roots which does not use the computationally heavy steps B1 and B4 in Algorithm B, by rather determining directly the information of step B6 (and then using of course the elementary results of Section 3). We will use for this the case by case descriptions of these lattices given by Venkov [44] or Conway and Sloane [18, Ch. 16], based on the classical connections between lattices and codes [18, 21], and study their automorphism groups in slightly more details than what we could find in the literature. To keep this section short, we assume some familiarity with Niemeier lattices and mostly follow the exposition in $[8$, Ch. 2.3] to which we refer for more details.

\subsection{Linking modules, Venkov modules and even unimodular lat-} tices. We first gather some definitions and notations.

(a) A (quadratic) linking module ${ }^{13}$ is a finite abelian group $A$ equipped with a quadratic map q $: A \rightarrow \mathbb{Q} / \mathbb{Z}$ whose associated symmetric $\mathbb{Z}$-bilinear map $\mathrm{b}(x, y):=\mathrm{q}(x+y)-\mathrm{q}(x)-\mathrm{q}(y), \quad A \times A \rightarrow \mathbb{Q} / \mathbb{Z}$, is a perfect pairing. The isometry group of $A$ is denoted $\mathrm{O}(A)$. If $I \subset A$ is a subgroup, we denote by $I^{\perp}$ the orthogonal of $I$ with respect to b. We say that $I$ is isotropic if we have $\mathrm{q}(I)=0$ (this is usually stronger than $\left.I \subset I^{\perp}\right)$. We say that $I$ is a Lagrangian if it is isotropic and if we have $I=I^{\perp}$ (or equivalently $|A|=|I|^{2}$ ).

(b) A Venkov module is a linking module $A$ equipped with a (set theoretic) map qm : $A \rightarrow \mathbb{Q}_{\geq 0}$ such that for all $a \in A$ we have $\mathrm{qm}(a) \equiv \mathrm{q}(a) \bmod \mathbb{Z}, \mathrm{qm}(0)=0$ and $\mathrm{qm}(a)>0$ for $a \neq 0$. Venkov modules form an additive category Ven in an obvious way; in particular we have an obvious notion of orthogonal direct sum of such objects, denoted $\oplus$. A root of a Venkov module $A$ is an element $a \in A$ such that $\mathrm{qm}(a)=1$.

(c) Assume $L$ is an even lattice in the Euclidean space $V$. Recall that we set $\mathrm{q}(x)=\frac{x \cdot x}{2}$ for $x \in V$. The finite abelian group $L^{\sharp} / L$, equipped with the well-defined quadratic map (that we shall still denote by q) $L^{\sharp} / L \rightarrow \mathbb{Q} / \mathbb{Z}, \quad x+L \mapsto \mathrm{q}(x) \bmod \mathbb{Z}$, is a linking module that we shall denote by res $L$ (sometimes also called the discriminant group or glue group of $L$ ). This linking module has a canonical structure

\footnotetext{
${ }^{13}$ Such a module is also called a qe-module in [8, Ch. 2.3].
} 
of Venkov module defined by

$$
\mathrm{qm}(x)=\inf _{y \in x+L} \mathrm{q}(y) .
$$

Let $\pi: L^{\sharp} \rightarrow$ res $L$ be the canonical projection. The map $I \mapsto \pi^{-1} I$ is a bijection between the set of isotropic subspaces $I$ of res $L$ and the set of even lattices of $V$ containing $L$. In this bijection, $\pi^{-1} I$ is unimodular if, and only if, $I$ is a Lagrangian. Moreover, we have $\mathrm{R}\left(\pi^{-1} I\right)=\mathrm{R}(L)$ if, and only if, $I$ does not contain any root of res $L$.

(d) We now focus on the case $L=\mathrm{Q}(R)$ with $R$ an ADE root system in $V$. In this case $\mathrm{Q}(R)^{\sharp}$ is called the weight lattice of $R$ and we set res $R=\operatorname{res} L$. The group $\mathrm{O}(R)$ naturally acts on res $R$, with $\mathrm{W}(R)$ acting trivially, so we have a morphism $\mathrm{G}(R) \rightarrow \operatorname{Aut}_{\text {Ven }}($ res $R$ ). The Venkov module res $R$ is the orthogonal sum of the res $S$ with $S$ an irreducible component of $R$. Assume now $R$ is irreducible. Canonical representatives for the nonzero elements of res $R$ are given by the so-called minuscule weights of $R$, that we denote by $\varpi_{i}$ following Bourbaki's conventions [4] for the indices. A key property is $\mathrm{qm}\left(\varpi_{i}+\mathrm{Q}(R)\right)=\mathrm{q}\left(\varpi_{i}\right)$ (see Table 5.1). We also have $\mathrm{G}(R) \stackrel{\sim}{\rightarrow} \operatorname{Aut}_{\mathrm{Ven}}($ res $R)$. In particular, the element - id of $\mathrm{O}(R)$ is in $\mathrm{W}(R)$ if, and only if, res $R$ is a $\mathbb{Z} / 2$-vector space.

TABLE 5.1. The Venkov module res $R$ for $R$ an irreducible

ADE root system.

\begin{tabular}{c|c|c|c|c|c|c}
$R$ & $\mathbf{A}_{n}$ & $\mathbf{D}_{n}, n$ even & $\mathbf{D}_{n}, n$ odd & $\mathbf{E}_{6}$ & $\mathbf{E}_{7}$ & $\mathbf{E}_{8}$ \\
\hline res $R$ & $\mathbb{Z} /(n+1)$ & $\mathbb{Z} / 2 \times \mathbb{Z} / 2$ & $\mathbb{Z} / 4$ & $\mathbb{Z} / 3$ & $\mathbb{Z} / 2$ & 0 \\
min.wts & $\varpi_{i}, i=1, \ldots, n$ & $\varpi_{n}, \varpi_{1}, \varpi_{n-1}$ & $\varpi_{n}, \varpi_{1}, \varpi_{n-1}$ & $\varpi_{1}, \varpi_{6}$ & $\varpi_{7}$ & \\
class & $i \bmod n+1$ & $\omega, 1, \bar{\omega}$ & $1,2,3 \bmod 4$ & $1,2 \bmod 3$ & $1 \bmod 2$ & \\
qm & $\frac{i(n+1-i)}{2(n+1)}$ & $\frac{n}{8}, \frac{1}{2}, \frac{n}{8}$ & $\frac{n}{8}, \frac{1}{2}, \frac{n}{8}$ & $\frac{2}{3}, \frac{2}{3}$ & $\frac{3}{4}$ &
\end{tabular}

Remark 5.2. In the case $R \simeq \mathbf{D}_{n}$ with $n$ even, some authors (e.g. [18]) identify the $\mathbb{Z} / 2$-vector space res $R$ with the finite field $\mathbb{F}_{4}=\{0,1, \omega, \bar{\omega}\}$. Using this identification, the automorphism group $\operatorname{Aut}_{\text {Ven }}($ res $R$ ) is generated by the Frobenius $f(x)=x^{2}$, as well as $m(x)=\omega x$ for $n=4$ (triality).

5.3. The Niemeier lattices with roots. Niemeier and Venkov have shown that $L \mapsto \mathrm{R}(L)$ induces a bijection between the isomorphism classes of Niemeier lattices with roots, and the isomorphism classes of equi-Coxeter ${ }^{14}$ ADE root systems in $\mathbb{R}^{24}$. Fix such a root system $R$ in

\footnotetext{
${ }^{14}$ A root system $R$ is called equi-Coxeter if its irreducible components have the same Coxeter number, then called the Coxeter number of $R$ and denoted $\mathrm{h}(R)$. The Coxeter numbers of $\mathbf{A}_{n}$, $\mathbf{D}_{n}, \mathbf{E}_{6}, \mathbf{E}_{7}$ and $\mathbf{E}_{8}$ are respectively $n+1,2 n-2,12,18$ and 30 .
} 
$\mathbb{R}^{24}$. By Section 5.1, there is thus a unique $\mathrm{O}(R)$-orbit of Lagrangians $I$ in res $R$ containing no root (the "codes"). For any such $I$, the associated Niemeier lattice with root system $R$ is $L=\pi^{-1} I$, we have $|\operatorname{res} R|=|I|^{2}$ and $\mathrm{G}(L)=\mathrm{O}(L) / \mathrm{W}(R)$ is the stabilizer of $I \subset$ res $R$ in $\mathrm{G}(R)$. In particular, the conjugacy class of $\mathrm{G}(L)$ in $\mathrm{G}(R)$ does not depend on the choice of $I$.

Goal. For each of the 23 possible isomorphism classes of $R$, determine the $\mathrm{G}(R)$-conjugacy class of the elements of $\mathrm{G}(L)$ (with their multiplicity).

This is exactly the information actually needed to apply Proposition 3.12 to each coset $\sigma \mathrm{W}(R)$ in $\mathrm{O}(L)$. We will use information on $\mathrm{G}(L)$ given by Venkov [44] and Conway-Sloane [18, Table 16.1] (see also [22]), such as their order and a composition series. Note that those $\mathrm{G}(L)$ are also exactly the umbral groups studied in [12]. ${ }^{15}$ We may assume that the decomposition of $R$ as a union of its irreducible components has the form ${ }^{16}$

$$
R=N_{1} R_{1} N_{2} R_{2} \ldots N_{g} R_{g}
$$

with $R_{i} \not R_{j}$ for $i \neq j$, and $N_{i} \geq 1$ for all $i$. We have natural decompositions

$$
\text { res } R=\bigoplus_{i}\left(\operatorname{res} R_{i}\right)^{N_{i}}, \mathrm{G}(R)=\prod_{i} \mathrm{G}\left(N_{i} R_{i}\right) \text { and } \mathrm{G}\left(N_{i} R_{i}\right)=\mathrm{G}\left(R_{i}\right) \imath \mathrm{S}_{N_{i}} \text {. }
$$

By (3.2), each $\mathrm{G}\left(N_{i} R_{i}\right)$ is naturally isomorphic either to the symmetric group $\mathrm{S}_{N_{i}}$, to the hyperoctahedral group $\mathrm{H}_{N_{i}}$, or to $\mathrm{T}_{N_{i}}:=\mathrm{S}_{3}<\mathrm{S}_{N_{i}}$ in the exceptional case $R_{i} \simeq \mathbf{D}_{4}$. The natural exact sequence $1 \rightarrow \prod_{i} \mathrm{G}\left(R_{i}\right)^{N_{i}} \rightarrow$ $\mathrm{G}(R) \rightarrow \prod_{i} \mathrm{~S}_{N_{i}} \rightarrow 1$ induces an exact sequence $1 \rightarrow \mathrm{G}_{1}(L) \rightarrow \mathrm{G}(L) \rightarrow$ $\mathrm{G}_{2}(L) \rightarrow 1$. The orders of $\mathrm{G}_{1}(L)$ and $\mathrm{G}_{2}(L)$ are given in [18, Table 16.1]. Moreover, the image of $\mathrm{G}_{2}(L)$ in $\mathrm{S}_{N_{i}}$ is always a transitive subgroup for each $i$.

We denote by $\eta \in \mathrm{G}(L)$ the class of the element - id of $\mathrm{O}(L)$. It is a central element which does not depend on the choice of $I$, and satisfies $\eta^{2}=1$. Its image in $\mathrm{G}\left(N_{i} R_{i}\right)$ is trivial if $R_{i}$ has type $\mathbf{A}_{1}, \mathbf{D}_{2 n}, \mathbf{E}_{7}$ or $\mathbf{E}_{8}$, and equal to the element -1 of $\mathrm{G}\left(N_{i} R_{i}\right)=\mathrm{H}_{N_{i}}$ otherwise (Section 2.1 and Table 5.1). An inspection of Table [18, Table 16.1] shows that we always have $\mathrm{G}_{1}(L)=\langle\eta\rangle$, except in the case $R \simeq \mathbf{D}_{4}^{6}$ for which we have $\mathrm{G}_{1}(L) \simeq$ $\mathbb{Z} / 3$ (and $\eta=1)$.

Notation. A conjugacy class $C \subset \mathrm{G}(R)$ has the form $\prod_{i} C_{i}$ where $C_{i}$ is a conjugacy class in $G_{i}=\mathrm{G}\left(N_{i} R_{i}\right)$. So $C$ is uniquely determined by the collection $\left(t_{i}\right)$ where $t_{i}$ is the type of $C_{i}$ : a partition of $N_{i}$ in the case $G_{i}=\mathrm{S}_{N_{i}}$, a couple of partitions as in Section 2.3 in the case $G_{i}=\mathrm{H}_{N_{i}}$, and similarly a triple of partitions in the case $G_{i}=\mathrm{T}_{N_{i}}$. In this last case, and as in Section 2.3, we use the sequence of symbols $\cdots i^{a_{i}} i^{b_{i}} i^{c_{i}} \ldots$ to denote the

\footnotetext{
${ }^{15}$ Although we will not use it, as this not the information we need, let us mention that the character tables of umbral groups have been listed in [12, Appendix 2] (and computed using GAP).

16 This is a short notation for $\coprod_{i=1}^{g} \coprod_{j=1}^{N_{i}} R_{i}$.
} 
conjugacy class whose elements have a cycle decomposition with $a_{i}$ (resp. $b_{i}, c_{i}$ ) cycles of length $i$ whose $i$-th power has order 1 (resp. 2, 3), with same conventions as loc.cit.

We now start the description of the $\mathrm{G}(R)$-conjugacy classes of the elements of $\mathrm{G}(L)$. In the non trivial cases, we list their type and give the number of elements of any given type divided by $|\mathrm{G}(L)|$ (the size of the type):

- $R \simeq \mathbf{D}_{24}, \quad \mathbf{D}_{16} \mathbf{E}_{8}, \mathbf{A}_{24}, \mathbf{A}_{17} \mathbf{E}_{7}, \quad \mathbf{A}_{15} \mathbf{D}_{9}$ and $\mathbf{A}_{11} \mathbf{D}_{7} \mathbf{E}_{6}$. We have $\mathrm{G}_{2}(L)=1$, so $\mathrm{G}(L)=\mathrm{G}_{1}(L)=\langle\eta\rangle$.

- $R \simeq 3 \mathbf{E}_{8}$. We have $\mathrm{G}(R)=\mathrm{S}_{3}$ and res $R=0$, so $\mathrm{G}(L)=\mathrm{G}(R)=\mathrm{S}_{3}$.

- $R \simeq 2 \mathbf{D}_{12}$. We have $\mathrm{G}(R)=\mathrm{H}_{2}, \mathrm{G}_{1}(L)=1$ and $\mathrm{G}_{2}(L)=\mathrm{S}_{2}$. We may take for $I$ the subgroup $\{0,(1, \omega),(\omega, 1),(\bar{\omega}, \bar{\omega})\}$ (note $\mathrm{q}(I)=$ $\{0,2,3\})$. For this $I, \mathrm{G}(L)$ is the natural subgroup $\mathrm{S}_{2}$ of $\mathrm{H}_{2}$.

- $R \simeq \mathbf{D}_{10} 2 \mathbf{E}_{7}$. We have $\mathrm{G}(R)=\mathrm{H}_{1} \times \mathrm{H}_{2}, \mathrm{G}_{1}(L)=1$ and $\mathrm{G}_{2}(L)=$ $\mathrm{S}_{2}$. We may take $I=\{0,(\omega, 1,0),(\bar{\omega}, 0,1),(1,1,1)\}($ note $\mathrm{qm}(I)=$ $\{0,2\})$. So $G(L) \simeq \mathbb{Z} / 2$ is generated by the element $\left(\varepsilon_{1},(12)\right)$ of $\mathrm{G}(R)$, whose type is $(1,2)$.

- $R \simeq 3 \mathbf{D}_{8}$. We have $\mathrm{G}(R)=\mathrm{H}_{3}, \mathrm{G}_{1}(L)=1$ and $\mathrm{G}_{2}(L)=\mathrm{S}_{3}$. We may take for $I$ the subgroup generated by the $\mathfrak{S}_{3}$-orbit of $(1,1, \omega)$ (it contains $(0, \bar{\omega}, \bar{\omega}),(\omega, \omega, \omega)$ and we have $|I|=8$ and $\operatorname{qm}(I)=$ $\{0,2,3\})$. For this $I, \mathrm{G}(L)$ is the natural subgroup $\mathrm{S}_{3}$ of $\mathrm{H}_{3}$.

- $R \simeq 2 \mathbf{A}_{12}$. We have $\mathrm{G}(R)=\mathrm{H}_{2}, \eta=-1$ and $\mathrm{G}_{2}(L)=\mathrm{S}_{2}$. We may take $I=\langle a\rangle \simeq \mathbb{Z} / 13$ with $a=(1,5)($ note $q m(I)=\{0,2,3\})$. The order 4 element $\sigma=\varepsilon_{1}(12)$ of $\mathrm{H}_{2}$ satisfies $\sigma a=-5 a$, hence generates $\mathrm{G}(L)$. The type of the elements of $\mathrm{G}(L)$ are thus $1^{2}, 1^{2}$ and 2 , with respective size $1 / 4,1 / 4$ and $1 / 2$.

- $R \simeq 4 \mathbf{E}_{6}$. We have $\mathrm{G}(R)=\mathrm{H}_{4}, \eta=-1$ and $\mathrm{G}_{2}(L)=\mathrm{S}_{4}: \mathrm{G}(L)$ is a central extension of $\mathrm{S}_{4}$ by $\mathbb{Z} / 2$. Let $I$ be the Lagrangian of res $R \simeq(\mathbb{Z} / 3)^{4}$ with $\pi^{-1} I=L$. The stabilizer of $I$ in $\mathrm{O}(\operatorname{res} R)$ is the semi-direct product of $\mathrm{GL}(I) \simeq \mathrm{GL}_{2}(\mathbb{Z} / 3)$ and of a $\mathbb{Z} / 3$-vector space. The natural morphism $\mathrm{G}(L) \rightarrow \mathrm{GL}(I)$ is thus injective, hence bijective. In particular, $\mathrm{G}(L)$ is not isomorphic to $\mathrm{S}_{4} \times \mathbb{Z} / 2$ and we are in case (ii.c) of Proposition 2.8: $\mathrm{G}(L)$ is $\mathrm{H}_{4}$-conjugate to the subgroup of Example 2.2. The $\mathrm{H}_{4}$-conjugacy classes of $\mathrm{G}(L)$ are thus given by Table 2.1 .

- $R \simeq 4 \mathbf{D}_{6}$. We have $\mathrm{G}(R)=\mathrm{H}_{4}, \mathrm{G}_{1}(L)=1$ and $\mathrm{G}_{2}(L)=\mathrm{S}_{4}$. We claim that $\mathrm{G}(L)$ does not contain the natural subgroup $\mathrm{S}_{4}$ of $\mathrm{H}_{4}$. Indeed, assume that the Lagrangian $I$ of res $R=\left(\operatorname{res} \mathrm{D}_{6}\right)^{4}$ defining $L$ is stable under $\mathrm{S}_{4}$. For any $x=\left(x_{1}, x_{2}, x_{3}, x_{4}\right) \in I$ and any $1 \leq i \neq j \leq 4$, we have $x+(i j) x$ in $I$, hence $2 \mathrm{qm}\left(x_{i}+x_{j}\right)$ is either 0 or an integer $\geq 2$. This forces $x_{i}+x_{j}=0$ by Table 5.1, hence $|I| \leq 4$ : a contradiction. By Proposition 2.8, the subgroup 
$\mathrm{G}(L) \subset \mathrm{H}_{4}$ is thus $\mathrm{H}_{4}$-conjugate to the subgroup $\mathrm{S}_{4}^{\epsilon}$, and we are done.

- $R \simeq 2 \mathbf{A}_{9} \mathbf{D}_{6}$. We have $\mathrm{G}(R)=\mathrm{H}_{2} \times \mathrm{H}_{1}, \eta=(-1,1)$ and $\mathrm{G}_{2}(L)=$ $\mathrm{S}_{2} \times \mathrm{S}_{1}$. We may take for $I$ the subgroup generated by the elements $a=(2,4,0), b=(5,0, \omega)$ and $c=(0,5, \bar{\omega})$, of respective orders 5 , 2 and $2($ check $q m(I)=\{0,2,3\})$. Observe that $I$ is stable by the element $\sigma=\left(\varepsilon_{2}(12),-1\right)$ of $\mathrm{G}(R)$ : we have $\sigma(a)=(4,-2,0)=2 a$, $\sigma(b)=(0,-5, \bar{\omega})=c$ and $\sigma(c)=(5,0, \omega)=b$. This shows $\mathrm{G}(L)=$ $\langle\sigma\rangle \simeq \mathbb{Z} / 4$, the types of its elements being $\left(1^{2}, 1\right),\left(1^{2}, 1\right)$ and $(2,1)$, with respective size $1 / 4,1 / 4$ and $1 / 2$.

- $R \simeq 3 \mathbf{A}_{8}$. We have $\mathrm{G}(R)=\mathrm{H}_{3}, \eta=-1$ and $\mathrm{G}_{2}(L)=\mathrm{S}_{3}$. By Proposition 2.8, $\mathrm{G}(L)$ is $\mathrm{H}_{3}$-conjugate to the subgroup $\{ \pm 1\} \cdot \mathrm{S}_{3}$ of $\mathrm{H}_{3}$.

- $R \simeq 2 \mathbf{A}_{7} 2 \mathbf{D}_{5}$. We have $\mathrm{G}(R)=\mathrm{H}_{2} \times \mathrm{H}_{2}, \eta=(-1,-1)$ and $\mathrm{G}_{2}(L)=$ $\mathrm{S}_{2} \times \mathrm{S}_{2}$. We may take for $I$ the subgroup generated by the elements $a=(1,1,1,2)$ and $b=(1,-1,2,1)$ of order $8(\operatorname{check} q \mathrm{qm}(I)=$ $\{0,2,3\})$. Note that $I$ is stable under $\sigma_{1}=\left((12), \varepsilon_{2}\right)$ and $\sigma_{2}=$ $\left(\varepsilon_{2},(12)\right)$ : we have $\sigma_{1}(a)=a, \sigma_{1}(b)=(-1,1,2,3)=-b$, and $\sigma_{2}$ exchanges $a$ and $b$. This shows $\mathrm{G}(L)=\left\langle\sigma_{1}, \sigma_{2}\right\rangle$ (dihedral of order $8)$, with types $\left(1^{2}, 1^{2}\right),\left(1^{2}, 1^{2}\right)$ of size $1 / 8$, and types $(2,11),(11,2)$ and $(2,2)$ of size $1 / 4$.

- $R \simeq 4 \mathbf{A}_{6}$. We have $\mathrm{G}(R)=\mathrm{H}_{4}, \eta=-1$ and $\left|\mathrm{G}_{2}(L)\right|=12$. So $\mathrm{G}(L)$ is a central extension of $\mathrm{G}_{2}(L)=\mathrm{Alt}_{4}$ by $\mathbb{Z} / 2$. It has an injective morphism to $\mathrm{GL}(I)=\mathrm{GL}_{2}\left(\mathbb{Z} / 7\right.$ ) (same argument as for $4 \mathbf{E}_{6}$ ): this is a non split extension. By Remark 2.9, the types of the elements of $\mathrm{G}(L)$ follow thus from Table 2.1: they are $1^{4}, 1^{4}, 2^{2}, 13$ and 13 , with respective sizes $1 / 24,1 / 24,1 / 4,1 / 3$ and $1 / 3$.

- $R \simeq 6 \mathbf{D}_{4}$. We have $\mathrm{G}(R)=\mathrm{T}_{6},\left|\mathrm{G}_{1}(L)\right|=3$ and $\mathrm{G}_{2}(L)=\mathrm{S}_{6}$. We identify res $\mathrm{D}_{4}$ with $\mathbb{F}_{4}$ as in Remark 5.2. Following Conway and Sloane, $I$ is an hexacode in $\mathbb{F}_{4}^{6}$. By $[18, \S 11.2]$, we may choose for $I$ the $\mathbb{F}_{4}$-vector space generated by the $K$-orbit of $(\omega, \bar{\omega}, \omega, \bar{\omega}, \omega, \bar{\omega})$, where $K$ is the subgroup of Alt $_{6}$ preserving $\{\{1,2\},\{3,4\},\{5,6\}\}$. For this choice, $\mathrm{G}(L)$ contains $K$ and $\mathrm{G}_{1}(L)$ is generated by the element $(m, m, m, m, m, m)$ of $\operatorname{Aut}_{\mathbb{Z} / 2}\left(\mathbb{F}_{4}\right)^{6}=\left(\mathrm{S}_{3}\right)^{6}$. Two other elements of $\mathrm{G}(L)$ are for instance $(f, f, f, f, f, f)(12)$ and $(1,1,1,1$, $\left.m^{2}, m\right)(123)$ (for the latter, recall that $(\omega, \omega, \bar{\omega}, \bar{\omega}, 1,1)$ is in $I$ ). As $K,(12)$ and (123) generate $\mathrm{S}_{6}$, a straightforward computation allows to list the types of the elements of $\mathrm{G}(L)$ : we obtain Table 5.2.

- $R \simeq 4 \mathbf{A}_{5} \mathbf{D}_{4}$. We have $\mathrm{G}(R)=\mathrm{H}_{4} \times \mathrm{T}_{1}, \eta=(-1,1)$ and $\mathrm{G}_{2}(L)=$ $\mathrm{S}_{4} \times \mathrm{S}_{1}$. We identify res $\mathrm{D}_{4}$ with $\mathbb{F}_{4}$ as in Remark 5.2. The first projection $\operatorname{pr}_{1}: \mathrm{G}(L) \rightarrow \mathrm{H}_{4}$ is injective, and its image $\mathrm{H}(L)$ is a central extension of $\mathrm{S}_{4}$ by $\mathbb{Z} / 2$. By Proposition $2.8, \mathrm{H}(L)$ is either 
TABLE 5.2. The $\mathrm{T}_{6}$-conjugacy classes of the elements of $3 . \mathrm{S}_{6}$.

\begin{tabular}{c|c|c|c|c|c|c|c|c} 
type & $1^{6}$ & $1^{6}$ & $1^{4} 2$ & $1^{2} 2^{2}$ & $1^{2} 2^{2}$ & $2^{3}$ & $11^{2} 3$ & $3^{2}$ \\
\hline size & $1 / 2160$ & $1 / 1080$ & $1 / 48$ & $1 / 48$ & $1 / 24$ & $1 / 48$ & $1 / 18$ & $1 / 18$ \\
\hline type & $1^{2} 4$ & 24 & 24 & 15 & 15 & 6 & 123 & \\
\hline size & $1 / 8$ & $1 / 24$ & $1 / 12$ & $2 / 15$ & $1 / 15$ & $1 / 6$ & $1 / 6$ &
\end{tabular}

conjugate to $\{ \pm 1\} \times \mathrm{S}_{4}$ or to the group $\mathrm{GL}_{2}(\mathbb{Z} / 3)$ embeded as in Example 2.2. By $[18, \S 11.2]$, we may take for $I$ the subgroup generated by the $\sigma$-orbit of $a=(2,0,2,4,0)$ and $b=(3,3,0,0, \bar{w})$, where $\sigma=((234), m)$. In order to determine $\mathrm{H}(L)$ it is enough to find the unique $\pm v \in\{ \pm 1\}^{4}$ such that $v(12) \in \mathrm{H}(L)$. Note that $2 I$ is the $\mathbb{Z} / 3$-vector space generated by $(2,0,2,4,0)$ and $(2,4,0,2,0)$. We deduce $v= \pm(1,1,1,-1)$ : we have $\mathrm{G}(L) \simeq \mathrm{H}(L) \simeq \mathrm{GL}_{2}(\mathbb{Z} / 3)$. On the other hand, the second projection $\mathrm{pr}_{2}: \mathrm{G}(L) \rightarrow \mathrm{T}_{1}$ is trivial on $\eta$ hence factors through a morphism $\mu: \mathrm{S}_{4} \rightarrow \mathrm{S}_{3}$. We have $\sigma \in \mathrm{G}(L)$ and $\operatorname{pr}_{2}(\sigma)=m$ has order 3: $\mu$ is "the" classical surjective morphism from $\mathrm{S}_{4}$ to $\mathrm{S}_{3}$. The types of $\mathrm{G}(L)$ are thus immediately deduced from Table 2.1.

- $R \simeq 6 \mathbf{A}_{4}$. We have $\mathrm{G}(R)=\mathrm{H}_{6}, \eta=-1$ and $\mathrm{G}_{2}(L)$ is a transitive subgroup of $\mathrm{S}_{6}$ of order 120 , so $\mathrm{G}_{2}(L)$ is isomorphic to $\mathrm{S}_{5}$ and $\mathrm{G}(L)$ is a central extension of $\mathrm{S}_{5}$ by $\mathbb{Z} / 2$ in $\mathrm{H}_{6}$. We claim that $\mathrm{G}(L)$ does not contain the triple transposition $\tau=(12)(34)(56)$. Indeed, otherwise the Lagrangian $I$ defining $L$ would be invariant by $\tau$. Set $I^{ \pm}=\{x \in I, \tau(x)= \pm x\}$. A nonzero element of $I^{+}$has the form $(a, a, b, b, c, c)$ with $2 \mathrm{qm}(a)+2 \mathrm{qm}(b)+2 \mathrm{qm}(c)$ an integer $\neq 1$. This forces $\{ \pm a, \pm b, \pm c\}=\mathbb{Z} / 5$ since $\mathrm{qm}\left(\operatorname{res} \mathrm{A}_{4}\right)=\left\{0, \frac{2}{5}, \frac{3}{5}\right\}$. But the nondegenerate conic $a^{2}+b^{2}+c^{2}=0$ in $(\mathbb{Z} / 5)^{3}$ contains all those vectors: we have $\operatorname{dim}_{\mathbb{Z} / 5} I^{+} \leq 1$. A similar argument shows $\operatorname{dim}_{\mathbb{Z} / 5} I^{-} \leq 1$. This is a contradiction as $I=I^{+} \oplus I^{-}$has dimension 3, hence the claim. By Proposition 2.10 and the discussion after this proposition, $\mathrm{G}(L)$ is $\mathrm{H}_{6}$-conjugate to the image of the map (2.2). The type of its elements are thus given by Table 2.2.

- $R \simeq 8 \mathbf{A}_{3}$. We have $\mathrm{G}(R)=\mathrm{H}_{8}, \eta=-1$ and $\left|\mathrm{G}_{2}(L)\right|=1344$. In this case, $I \subset$ res $R=(\mathbb{Z} / 4)^{8}$ is a so-called octacode [18]. The subgroup $C:=I / 2 I$ a Hamming code in res $R \otimes \mathbb{Z} / 2=(\mathbb{Z} / 2)^{8}$ and $\mathrm{G}_{2}(L)$ is the automorphism group of this code. In particular, $C$ is included in the hyperplane $H$ of $(\mathbb{Z} / 2)^{8}$ defined by $\sum_{i} x_{i}=0$ and $V:=H / C$ is a hyperplane in the 4 -dimensional $\mathbb{Z} / 2$-vector space $W:=(\mathbb{Z} / 2)^{8} / C$. The (easy) theory of Hamming codes shows that the map $\iota:\{1, \ldots, 8\} \rightarrow W$, sending $j$ to the class of the 
canonical basis element $\delta_{j}$ of $(\mathbb{Z} / 2)^{8}$, is injective with image an affine hyperplane under $V$, and identifies $\mathrm{G}_{2}(L)$ with the affine group of $\{1, \ldots, 8\}$ for this affine structure. In particular, $\mathrm{G}_{2}(L)$ is isomorphic to $\mathrm{GA}_{3}(\mathbb{Z} / 2)=(\mathbb{Z} / 2)^{3} \rtimes \mathrm{GL}_{3}(\mathbb{Z} / 2)$. To go further we choose some $I$ : following [18, Table 16.1] we take the subgroup generated by the $c$-orbit of the element $(3,2,0,0,1,0,1,1)$ where $c$ is the 7-cycle $(2345678)$ (we have $\mathrm{qm}(I)=\{0,2,3,4\})$. With this choice of $I$, we have $c \in \mathrm{G}(L)$ and checks that $\tau=(346)(587)$ and $\sigma=\varepsilon_{3} \varepsilon_{6} \varepsilon_{7} \varepsilon_{8}(23)(4568)$ lie in $\mathrm{G}(L)$ as well. The images in $\mathrm{G}_{2}(L)$ of $1, c, c^{-1}, \tau, \sigma$ and $\sigma^{2}$ are representatives of the conjugacy classes of the stabilizer $\mathrm{G}_{2}(L)_{1}$ of 1 in $\{1, \ldots, 8\}$, with resp. sizes $1 / 168,1 / 7$, $1 / 7,1 / 3,1 / 4$ and $1 / 8\left(\right.$ recall $\mathrm{G}_{2}(L)_{1} \simeq \mathrm{GL}_{3}(\mathbb{Z} / 2)$ ). But $c, \tau$ and $\sigma$ belong to the stabilizer $\mathrm{G}(L)_{1}$ of $1 \in\{ \pm 1\}^{8}$ in $\mathrm{G}(L)$ : the natural map $\mathrm{G}(L) \rightarrow \mathrm{G}_{2}(L)$ induces an isomorphism $\mathrm{G}(L)_{1} \stackrel{\sim}{\rightarrow} \mathrm{G}_{2}(L)_{1}$. An inspection of $C$ shows that the translation by the class of $\delta_{1}-\delta_{2}$ in $V$ is the element (12) (37) (45) (68) of $\mathrm{S}_{8}$. One deduces from these information representatives of the conjugacy classes of $\mathrm{G}(L)$ : their types are gathered in Table 5.3.

TABLE 5.3. The $\mathrm{H}_{8}$-conjugacy classes of the nontrivial elements of $2 . \mathrm{GA}_{3}(\mathbb{Z} / 2)$.

\begin{tabular}{c|c|c|c|c|c|c|c|c|c|c|c|c} 
type & $1^{8}$ & $1^{2} 1^{2} 2^{2}$ & $2^{4}$ & $2^{4}$ & $1^{2} 3^{2}$ & $1^{2} 3^{2}$ & 1124 & $4^{2}$ & $4^{2}$ & 26 & 17 & 17 \\
\hline size & $1 / 2688$ & $1 / 32$ & $1 / 192$ & $1 / 32$ & $1 / 12$ & $1 / 12$ & $1 / 8$ & $1 / 16$ & $1 / 8$ & $1 / 6$ & $1 / 7$ & $1 / 7$
\end{tabular}

- $R \simeq 12 \mathbf{A}_{2}$. We have $\mathrm{G}(R)=\mathrm{H}_{12}, \eta=-1$ and $\mathrm{G}_{2}(L)$ is isomorphic to the Mathieu group $\mathrm{M}_{12}$. The Lagrangian $I \subset \operatorname{res} R=(\mathbb{Z} / 3)^{12}$ is a ternary Golay code, whose automorphism group $\mathrm{G}(L)$ is the central extension of $\mathrm{M}_{12}$ by $\mathbb{Z} / 2$ denoted $2 . \mathrm{M}_{12}$ in the $\mathbb{A} \mathbb{T} \mathbb{L} \mathbb{A}$. We know since Frobenius [24, p. 11] the cycle decompositions, and cardinality, of all the conjugacy classes of $\mathrm{M}_{12}$. The inverse image in $2 . \mathrm{M}_{12}$ of such a class $c$ is the union of one or two conjugacy classes $c^{\prime} \cup-c^{\prime}$, the cycle decomposition of $c^{\prime}$ being the same as that of $c$ except that each cycle of $c$ now has a sign to be determined. It is an amusing exercise $^{17}$ to extract these signs from the lines $\chi_{2}$ and $\chi_{18}$, and from the power maps, of the character table of $2 . \mathrm{M}_{12}$ in the $\mathbb{A} \mathbb{T} L \mathbb{A} S$. We obtain Table $5.4 .^{18}$

\footnotetext{
17 That such an exercise is possible follows from the following fact: if we have an equality of

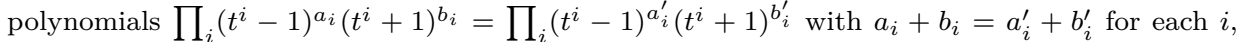
then $a_{i}=a_{i}^{\prime}$ and $b_{i}=b_{i}^{\prime}$ for each $i$ (use $t^{i}+1=\left(t^{2 i}-1\right) /\left(t^{i}-1\right)$ and Lemma 3.3).

${ }^{18}$ An alternative way to proceed is to use the description of $2 . \mathrm{M}_{12}$ given by Hall in [29], as the automorphism group of a $12 \times 12$ Hadamard matrix (a subgroup of $\mathrm{H}_{12}$ ). Using the 4 generators given by Hall loc. cit., and applying GAP's ConjugacyClasses algorithm to the permutation group on 24 letters they generate, we confirm Table 5.4.
} 
TABLE 5.4. The $\mathrm{H}_{12}$-conjugacy classes of the nontrivial elements of $2 . \mathrm{M}_{12}$.

\begin{tabular}{c|c|c|c|c|c|c|c|c|c|c} 
type & $1^{12}$ & $2^{6}$ & $1^{4} 2^{4}$ & $1^{4} 2^{4}$ & $1^{3} 3^{3}$ & $1^{3} 3^{3}$ & $3^{4}$ & $3^{4}$ & $2^{2} 4^{2}$ & $1^{2} 1^{2} 4^{2}$ \\
\hline size & $1 / 190080$ & $1 / 240$ & $1 / 384$ & $1 / 384$ & $1 / 108$ & $1 / 108$ & $1 / 72$ & $1 / 72$ & $1 / 32$ & $1 / 32$ \\
\hline type & $1^{2} 5^{2}$ & $1^{2} 5^{2}$ & $6^{2}$ & 1236 & 1236 & 48 & 1128 & 210 & 111 & 111 \\
\hline size & $1 / 20$ & $1 / 20$ & $1 / 12$ & $1 / 12$ & $1 / 12$ & $1 / 8$ & $1 / 8$ & $1 / 10$ & $1 / 11$ & $1 / 11$
\end{tabular}

- $R \simeq 24 \mathbf{A}_{1}$. We have $\mathrm{G}(R)=\mathrm{S}_{24}$ and $\mathrm{G}(L)$ is a Mathieu group $\mathrm{M}_{24}$. The cycle decompositions and cardinality of the conjugacy classes of $\mathrm{M}_{24}$ are given by Frobenius in [24, p. 12-13]: see Table 5.5.

TABLE 5.5. The $\mathrm{S}_{24}$-conjugacy classes of the nontrivial elements of $\mathrm{M}_{24}$.

\begin{tabular}{c|c|c|c|c|c|c|c|c|c|c} 
type & $1^{8} 2^{8}$ & $2^{12}$ & $1^{6} 3^{6}$ & $3^{8}$ & $2^{4} 4^{4}$ & $1^{4} 2^{2} 4^{4}$ & $4^{6}$ & $1^{4} 5^{4}$ & $1^{2} 2^{2} 3^{2} 6^{2}$ & $6^{4}$ \\
\hline mass & $1 / 21504$ & $1 / 7680$ & $1 / 1080$ & $1 / 504$ & $1 / 384$ & $1 / 128$ & $1 / 96$ & $1 / 60$ & $1 / 24$ & $1 / 24$ \\
\hline type & $1^{3} 7^{3}$ & $1^{2} 248^{2}$ & $2^{2} 10^{2}$ & $1^{2} 11^{2}$ & 24612 & $12^{2}$ & 12714 & 13515 & 321 & 123 \\
\hline mass & $1 / 21$ & $1 / 16$ & $1 / 20$ & $1 / 11$ & $1 / 12$ & $1 / 12$ & $1 / 7$ & $2 / 15$ & $2 / 21$ & $2 / 23$
\end{tabular}

Comparison with the output of Algorithm B. For each of the 23 root systems $R$ above, we verified that the types and sizes of the $\mathrm{G}(R)$-conjugacy classes of $\mathrm{G}(L)$ found are exactly those returned (from scratch, and in a few seconds!) by Algorithm B (components 3 and 4 returned by masses_calc, see Section 4.3). The natural isomorphism $\mathrm{O}(L)_{\rho} \simeq \mathrm{G}(L)$ and Algorithm $\mathrm{B}$ provide thus a rather useful tool to study the groups $\mathrm{G}(L)$.

\section{Appendix A. Irreducible characters of compact orthogonal groups}

Let $n \geq 1$ be an integer. We denote by $\mathrm{O}(n)$ the isometry group of the standard Euclidean space $V=\mathbb{R}^{n}$. We know since Weyl that the complex, irreducible, continuous representations of the compact group $\mathrm{O}(n)$ are all defined over $\mathbb{R}$ and parameterized in a natural way by the $n$-permissible (integer) partitions $\lambda$. In this section, we recall this parameterization and discuss formulas for the irreducible characters due to Weyl and KoikeTerrada.

A.1. The $\boldsymbol{n}$-permissible partitions. Recall that a partition $\lambda$ is a nonincreasing integer sequence $\lambda_{1} \geq \lambda_{2} \geq \cdots$ with $\lambda_{i} \geq 0$ for all $i \geq 1$ and $\lambda_{i}=0$ for $i$ big enough. We also say that $\lambda$ is a partition of the integer $|\lambda|:=\sum_{i} \lambda_{i}$. The diagram of $\lambda$ is the Young diagram whose $i$-th row has 
$\lambda_{i}$ boxes for each $i \geq 1$. The dual of $\lambda$ is the partition $\lambda^{*}$ defined by $\lambda_{i}^{*}=\left|\left\{j \geq 1 \mid \lambda_{j} \geq i\right\}\right|$ (with "transpose" diagram).

Following Weyl, the partition $\lambda$ is called $n$-permissible if the first two columns of its diagram contain at most $n$ boxes, or equivalently if we have $\lambda_{1}^{*}+\lambda_{2}^{*} \leq n$. If $\lambda$ is $n$-permissible, there is a unique $n$-permissible partition $\mu$ with $\lambda_{i}^{*}=\mu_{i}^{*}$ for $i>1$ and $\lambda_{1}^{*}+\mu_{1}^{*}=n$, called the associate of $\lambda$ and denoted $\operatorname{ass}(\lambda)$. The map $\lambda \mapsto \operatorname{ass}(\lambda)$ is an involution of the set of $n$-permissible integer partitions.

An partition $\lambda$ is called $n$-positive if we have $\lambda_{1}^{*} \leq n / 2$ (hence $\lambda_{i}=0$ for $i>n / 2)$. If $\lambda$ is $n$-admissible but not $n$-positive, then $\operatorname{ass}(\lambda)$ is $n$-positive.

A.2. Weyl's construction. For any integer $d \geq 0$, we consider following Weyl the kernel $\mathrm{K}_{d}(V)$ of the direct sum of the $d(d-1) / 2$ contraction maps $^{19}$

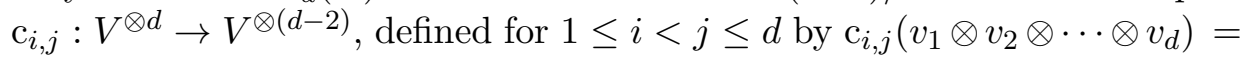
$\left(v_{i} \cdot v_{j}\right) v_{1} \otimes v_{2} \otimes \cdots \otimes \widehat{v}_{i} \otimes \cdots \otimes \widehat{v_{j}} \otimes \cdots \otimes v_{d}$. This kernel has a natural linear action of $\mathrm{O}(n) \times \mathfrak{S}_{d}$, hence decomposes as

$$
\mathrm{K}_{d}(V) \simeq \bigoplus_{\{\lambda|| \lambda \mid=d\}} \mathrm{K}_{\lambda}(V) \otimes \mathrm{R}_{\lambda}
$$

where $\mathrm{R}_{\lambda}$ is "the" irreducible representation of $\mathfrak{S}_{d}$ classically parameterized by $\lambda$, and $\mathrm{K}_{\lambda}(V)$ is a real representation of $\mathrm{O}(n)$. Set $\mathrm{W}_{\lambda}=\mathrm{K}_{\lambda}(V) \otimes \mathbb{C}$.

Weyl shows that $\mathrm{W}_{\lambda}$ is either 0 or an irreducible representation of $\mathrm{O}(n)$ [46, Thm. 5.7.D]. Moreover, $\mathrm{W}_{\lambda}$ is nonzero if and only if $\lambda$ is $n$-permissible [46, Thm. 5.7.A \& C]. Moreover, he shows that $\lambda \rightarrow \mathrm{W}_{\lambda}$ is a bijection between the set of $n$-permissible partitions and the isomorphism classes of irreducible representations of $\mathrm{O}(n)$ [46, Thm. 5.7.H \& 7.9.B]. The element $-1_{n}$ clearly acts as multiplication by $(-1)^{d}$ on $\mathrm{W}_{\lambda}$. Weyl shows

$$
\mathrm{W}_{\mathrm{ass}(\lambda)} \simeq \mathrm{W}_{\lambda} \otimes \operatorname{det}
$$

and studies the restriction of $\mathrm{W}_{\lambda}$ to the index two subgroup $\mathrm{SO}(n) \subset \mathrm{O}(n)$ in Chap. V.9 \& VII.9. We may assume $\lambda$ is $n$-positive. There are two cases:

(i) $\lambda \neq \operatorname{ass}(\lambda)$. The restriction of $\mathrm{W}_{\lambda}$ to $\mathrm{SO}(n)$ is then irreducible with highest weight $\sum_{i \leq n / 2} \quad \lambda_{i} \varepsilon_{i}$, using the classical notations of Bourbaki [4, Pl. IV]. Moreover, the natural action of $\mathrm{O}(n) / \mathrm{SO}(n)=$ $\mathbb{Z} / 2$ on the highest weight lines of $\mathrm{W}_{\lambda}$ is trivial (and non trivial on those of $\left.\mathrm{W}_{\mathrm{ass}(\lambda)} \otimes \mathbb{C}\right)$.

(ii) $\lambda=\operatorname{ass}(\lambda)$. This forces $n \equiv 0 \bmod 2$ and $\lambda_{n / 2}>0$. The restriction of $\mathrm{W}_{\lambda}$ to $\mathrm{SO}(n)$ is then the sum of the two irreducible representations, conjugate under $\mathrm{O}(n)$, with highest weights $\pm \lambda_{n / 2} \varepsilon_{n / 2}+$ $\sum_{i=1}^{n / 2-1} \lambda_{i} \varepsilon_{i}$

\footnotetext{
${ }^{19}$ All tensor products are taken over $\mathbb{R}$ in Section A.2.
} 
A.3. Character formulas. Weyl gives a determinantal formula for the character of $\mathrm{W}_{\lambda}$ in [46, Thm. 7.9.A]. Contrary to the standard so-called Weyl character formula, which applies to any connected compact Lie groups, that formula equally applies to elements in any of the two connected components ${ }^{20}$ of $\mathrm{O}(n)$. Assume $g$ is in $\mathrm{O}(n)$ and write $\operatorname{det}(1-t g)^{-1}=$ $\sum_{i \in \mathbb{Z}} p_{i} t^{i}$ in $\mathbb{Z}[[t]]$ (so $p_{i}=0$ for $\left.i<0\right)$. Weyl shows loc. cit. that for any $n$-permissible partitions $\lambda$ we have

$$
\operatorname{Trace}\left(g ; \mathrm{W}_{\lambda}\right)=\operatorname{det}\left(p_{\lambda_{i}-i+j}-p_{\lambda_{i}-i-j}\right)_{1 \leq i, j \leq \lambda_{1}^{*}} .
$$

If we write $\operatorname{det}(1+t g)=\sum_{i \in \mathbb{Z}} e_{i} t^{i}$ (so $e_{i}=0$ for $i<0$ or $i>n$ ), and set $\delta_{1}=0$ and $\delta_{j}=1$ for $j>1$, then [33, Thm. 2.3.3(6)] implies

$$
\text { (A.3) } \operatorname{det}\left(p_{\lambda_{i}-i+j}-p_{\lambda_{i}-i-j}\right)_{1 \leq i, j \leq \lambda_{1}^{*}}=\operatorname{det}\left(e_{\lambda_{i}^{*}-i+j}+\delta_{j} e_{\lambda_{i}^{*}-i-j+2}\right)_{1 \leq i, j \leq \lambda_{1}} \text {, }
$$

See also the equivalence of (ii) and (iv) in [25, Cor. A.46] for a direct alternative proof of this equality.

Remark A.4. In the case $\lambda_{1}=0$, or equivalently $|\lambda|=0$ or $\lambda$ is the empty diagram, then $\mathrm{W}_{\lambda}$ is the trivial representation and both determinants above are indeed 1 by convention. Moreover, the formula $e_{n-i}=(\operatorname{det} g) e_{i}$ for $i \in \mathbb{Z}$ shows that the determinant on the right-hand side of (A.3) is multiplied by $\operatorname{det} g$ if $\lambda$ is replaced by ass $(\lambda)$ (it amounts to multiply by $\operatorname{det} g$ the first line of the matrix inside the determinant), in agreement with Formula (A.1).

\section{Appendix B. An asymptotic formula}

Proposition B.1. Let $L$ be a lattice in the Euclidean space $\mathbb{R}^{n}$ and $\lambda$ an $n$-permissible partition with $|\lambda| \equiv 0 \bmod 2$. Then we have

$$
\operatorname{dim} \mathrm{W}_{\lambda}^{\mathrm{O}(L)} \sim \frac{2}{|\mathrm{O}(L)|} \operatorname{dim} \mathrm{W}_{\lambda}
$$

for $\lambda \rightarrow \infty$, in the sense that $\lambda_{i}-\lambda_{i+1} \rightarrow+\infty$ for each $1 \leq i \leq n / 2$.

Proof. As we have $\lambda \rightarrow \infty$ we may assume $\lambda$ is positive and $\lambda_{[n / 2]}>$ 0 . Denote by $\mathrm{V}_{\lambda}$ the irreducible constituent of $\left(\mathrm{W}_{\lambda}\right)_{\mid \mathrm{SO}(n)}$ with highest weight $\sum_{i \leq n / 2} \lambda_{i} \varepsilon_{i}$. Set $\mathrm{SO}(L)=\mathrm{O}(L) \cap \mathrm{SO}(n)$. If $n$ is odd, we have $\left(\mathrm{W}_{\lambda}\right)_{\mid \mathrm{SO}(n)}=\mathrm{V}_{\lambda}, \mathrm{O}(L)=\{ \pm \mathrm{id}\} \times \mathrm{SO}(L)$ and $\mathrm{W}_{\lambda}^{\mathrm{O}(L)}=\mathrm{V}_{\lambda}^{\mathrm{SO}(L)}$. If $n$ is even, then $\left(\mathrm{W}_{\lambda}\right)_{\mid \mathrm{SO}(n)}$ is the direct sum of $\mathrm{V}_{\lambda}$ and of its outer conjugate $\mathrm{V}_{\lambda}^{\prime}$, and $\mathrm{W}_{\lambda}$ is induced from $\mathrm{V}_{\lambda}$ : we have thus $\mathrm{W}_{\lambda}^{\mathrm{O}(L)}=\mathrm{V}_{\lambda}^{\mathrm{SO}(L)}$ in the case $\mathrm{O}(L) \neq \mathrm{SO}(L)$ and $\mathrm{W}_{\lambda}^{\mathrm{O}(L)}=\mathrm{V}_{\lambda}^{\mathrm{SO}(L)} \oplus\left(\mathrm{V}_{\lambda}^{\prime}\right)^{\mathrm{SO}(L)}$ otherwise. We conclude from the degenerate form of Weyl's character formula for $\mathrm{SO}(n)$ given in $[7$, Prop. 1.9].

\footnotetext{
${ }^{20}$ Let us mention that there exists also a variant of the Weyl character formula which applies to the irreducible characters of non connected compact Lie groups: see e.g. [34, 45].
} 
Assume now $n \equiv-1,0,1 \bmod 8$ and set $\mu_{n}=\sum_{[L] \in \mathrm{X}_{n}} \frac{1}{|\mathrm{O}(L)|}$. The mass formula of Minkowski-Siegel-Smith asserts that we have $\mu_{n}=$ $\left|\frac{\mathrm{B}_{n / 2}}{n} \prod_{j=1}^{n / 2-1} \frac{\mathrm{B}_{2 j}}{4 j}\right|$ for $n \equiv 0 \bmod 8$, and $\mu_{n}=\left|\prod_{j=1}^{(n-1) / 2} \frac{\mathrm{B}_{2 j}}{4 j}\right|$ for $n \equiv \pm 1 \bmod$ 8 , where the $\mathrm{B}_{m}$ are the Bernouilli numbers [17].

Corollary B.2. For $n \equiv-1,0,1 \bmod 8,|\lambda| \equiv 0 \bmod 2$ and $\lambda \rightarrow \infty$ we have $\operatorname{dim} \mathrm{M}_{\mathrm{W}_{\lambda}}\left(\mathrm{O}_{n}\right) \sim 2 \mu_{n} \operatorname{dim} \mathrm{W}_{\lambda}$.

For instance, in the case $n=24$ of main interest here we have $\mu_{24} \approx$ $8 \cdot 10^{-15}$, quite a small number compared to $\left|\mathrm{X}_{24}\right|=24$, and of course we expect $\operatorname{dim} \mathrm{M}_{W_{\lambda}}\left(\mathrm{O}_{24}\right)$ to be small for small values of $\lambda$.

\section{Appendix C. Tables}

In the following tables, we use the notation $1^{a_{1}} 2^{a_{2}} \ldots m^{a_{m}}$ for the polynomial $\varphi_{1}^{a_{1}} \varphi_{2}^{a_{2}} \cdots \varphi_{m}^{a_{m}}$, where $\varphi_{n}$ is the $n$-th cyclotomic polynomial and where the symbol " $i$ " is omitted for $a=0$, and shorten as " $i$ " for $a=1$.

TABle C.1. The 7 nonzero $m=\mathrm{m}_{\sigma \mathrm{W}\left(\mathrm{D}_{4}\right)}(P)$ for $P$ in $\mathrm{Car}_{4}$, where $\sigma$ in $\mathrm{G}\left(\mathrm{D}_{4}\right)$ has order 3 .

\begin{tabular}{c|c|c|c|c|c|c|c|c|c|c|c|c|c}
$P$ & $m$ & $P$ & $m$ & $P$ & $m$ & $P$ & $m$ & $P$ & $m$ & $P$ & $m$ & $P$ & $m$ \\
\hline $3^{2}$ & $1 / 24$ & $6^{2}$ & $1 / 24$ & $1^{2} 3$ & $1 / 12$ & $2^{2} 6$ & $1 / 12$ & 123 & $1 / 4$ & 12 & $1 / 4$ & 126 & $1 / 4$
\end{tabular}

Table C.2. The 25 nonzero $m=\mathrm{m}_{\mathrm{W}\left(\mathrm{E}_{6}\right)}(P)$ for $P$ in $\mathrm{Car}_{6}$.

\begin{tabular}{c|c|c|c|c|c|c|c|c|c}
$P$ & $m$ & $P$ & $m$ & $P$ & $m$ & $P$ & $m$ & $P$ & $m$ \\
\hline $1^{6}$ & $1 / 51840$ & $1^{4} 2^{2}$ & $1 / 192$ & $36^{2}$ & $1 / 72$ & $1^{3} 24$ & $1 / 32$ & 312 & $1 / 12$ \\
$1^{5} 2$ & $1 / 1440$ & $1^{2} 3^{2}$ & $1 / 108$ & $1^{3} 23$ & $1 / 36$ & $1^{2} 2^{2} 3$ & $1 / 24$ & $1^{2} 5$ & $1 / 10$ \\
$1^{2} 2^{4}$ & $1 / 1152$ & $1^{2} 4^{2}$ & $1 / 96$ & $1^{2} 2^{2} 6$ & $1 / 36$ & $1^{2} 2^{2} 4$ & $1 / 16$ & 125 & $1 / 10$ \\
$3^{3}$ & $1 / 648$ & $1^{3} 2^{3}$ & $1 / 96$ & $123^{2}$ & $1 / 36$ & 1246 & $1 / 12$ & 9 & $1 / 9$ \\
$1^{4} 3$ & $1 / 216$ & $12^{3} 4$ & $1 / 96$ & $2^{2} 36$ & $1 / 36$ & 1236 & $1 / 12$ & 128 & $1 / 8$
\end{tabular}


TABle C.3. The 54 nonzero $m=\mathrm{m}_{\mathrm{W}\left(\mathrm{E}_{7}\right)}(P)$ for $P$ in $\mathrm{Car}_{7}$.

\begin{tabular}{c|c|c|c|c|c|c|c|c|c|c|c}
$P$ & $m$ & $P$ & $m$ & $P$ & $m$ & $P$ & $m$ & $P$ & $m$ & $P$ & $m$ \\
\hline $1^{7}$ & $1 / 2903040$ & $26^{3}$ & $1 / 1296$ & $1^{2} 2^{3} 3$ & $1 / 288$ & $1^{2} 2^{3} 6$ & $1 / 96$ & $1^{2} 246$ & $1 / 48$ & 2612 & $1 / 24$ \\
$2^{7}$ & $1 / 2903040$ & $1^{3} 4^{2}$ & $1 / 768$ & $1^{4} 23$ & $1 / 288$ & $12^{2} 6^{2}$ & $1 / 72$ & $1^{2} 234$ & $1 / 48$ & $12^{2} 10$ & $1 / 20$ \\
$12^{6}$ & $1 / 46080$ & $2^{3} 4^{2}$ & $1 / 768$ & $12^{2} 4^{2}$ & $1 / 256$ & $1^{2} 23^{2}$ & $1 / 72$ & $12^{2} 8$ & $1 / 32$ & $1^{2} 25$ & $1 / 20$ \\
$1^{6} 2$ & $1 / 46080$ & $1^{3} 2^{4}$ & $13 / 9216$ & $1^{2} 24^{2}$ & $1 / 256$ & $1^{3} 5$ & $1 / 60$ & 148 & $1 / 32$ & $12^{2} 36$ & $1 / 18$ \\
$1^{5} 3$ & $1 / 4320$ & $1^{4} 2^{3}$ & $13 / 9216$ & $1^{3} 3^{2}$ & $1 / 216$ & $2^{3} 10$ & $1 / 60$ & $1^{2} 28$ & $1 / 32$ & 19 & $1 / 18$ \\
$2^{5} 6$ & $1 / 4320$ & $12^{4} 4$ & $1 / 384$ & $2^{3} 6^{2}$ & $1 / 216$ & $1^{3} 2^{2} 4$ & $7 / 384$ & 248 & $1 / 32$ & $1^{2} 236$ & $1 / 18$ \\
$1^{5} 2^{2}$ & $1 / 3072$ & $1^{4} 24$ & $1 / 384$ & $136^{2}$ & $1 / 144$ & $1^{2} 2^{3} 4$ & $7 / 384$ & 135 & $1 / 30$ & 218 & $1 / 18$ \\
$1^{2} 2^{5}$ & $1 / 3072$ & $1^{3} 2^{2} 6$ & $1 / 288$ & $23^{2} 6$ & $1 / 144$ & $12^{2} 34$ & $1 / 48$ & 2610 & $1 / 30$ & 17 & $1 / 14$ \\
$13^{3}$ & $1 / 1296$ & $12^{4} 6$ & $1 / 288$ & $1^{3} 2^{2} 3$ & $1 / 96$ & $12^{2} 46$ & $1 / 48$ & 1312 & $1 / 24$ & 214 & $1 / 14$
\end{tabular}

TABle C.4. The 106 nonzero $m=\mathrm{m}_{\mathrm{W}\left(\mathrm{E}_{8}\right)}(P)$ for $P$ in $\mathrm{Car}_{8}$.

\begin{tabular}{|c|c|c|c|c|c|c|c|c|c|}
\hline$P$ & $m$ & $P$ & $m$ & $P$ & $m$ & $P$ & $m$ & $P$ & $m$ \\
\hline $1^{8}$ & $1 / 696729600$ & $1^{2} 2^{4} 3$ & $1 / 6912$ & $1^{3} 2^{3} 6$ & $1 / 576$ & $2^{2} 612$ & $1 / 144$ & 12312 & $1 / 48$ \\
\hline $2^{8}$ & $1 / 696729600$ & $1^{4} 2^{4}$ & $37 / 221184$ & $1^{3} 246$ & $1 / 576$ & $1^{2} 48$ & $1 / 128$ & 1268 & $1 / 48$ \\
\hline $12^{7}$ & $1 / 5806080$ & $123^{3}$ & $1 / 2592$ & $1^{2} 34^{2}$ & $1 / 576$ & $2^{2} 48$ & $1 / 128$ & 12612 & $1 / 48$ \\
\hline $1^{7} 2$ & $1 / 5806080$ & $126^{3}$ & $1 / 2592$ & $2^{2} 4^{2} 6$ & $1 / 576$ & $12^{3} 10$ & $1 / 120$ & 1245 & $1 / 40$ \\
\hline $1^{6} 3$ & $1 / 311040$ & $1^{4} 3^{2}$ & $1 / 2592$ & $12^{3} 6^{2}$ & $1 / 432$ & $1^{3} 25$ & $1 / 120$ & 12410 & $1 / 40$ \\
\hline $2^{6} 6$ & $1 / 311040$ & $2^{4} 6^{2}$ & $1 / 2592$ & $1^{3} 23^{2}$ & $1 / 432$ & $1^{2} 2^{2} 4^{2}$ & $9 / 1024$ & 129 & $1 / 36$ \\
\hline $1^{6} 2^{2}$ & $1 / 184320$ & $3^{2} 6^{2}$ & $1 / 1728$ & $12^{3} 8$ & $1 / 384$ & $1^{2} 9$ & $1 / 108$ & 1218 & $1 / 36$ \\
\hline $1^{2} 2^{6}$ & $1 / 184320$ & $12^{3} 4^{2}$ & $1 / 1536$ & $1^{3} 28$ & $1 / 384$ & $2^{2} 18$ & $1 / 108$ & $1^{2} 2^{2} 36$ & $1 / 36$ \\
\hline $6^{4}$ & $1 / 155520$ & $1^{3} 24^{2}$ & $1 / 1536$ & $123^{2} 6$ & $1 / 288$ & $1^{2} 2^{2} 46$ & $1 / 96$ & 15 & $1 / 30$ \\
\hline $3^{4}$ & $1 / 155520$ & $1^{4} 5$ & $1 / 1200$ & $1236^{2}$ & $1 / 288$ & $1^{2} 2^{2} 34$ & $1 / 96$ & 30 & $1 / 30$ \\
\hline $4^{4}$ & $1 / 46080$ & $2^{4} 10$ & $1 / 1200$ & $1^{2} 2^{2} 6^{2}$ & $1 / 288$ & $1^{2} 2^{2} 10$ & $1 / 80$ & 127 & $1 / 28$ \\
\hline $1^{3} 2^{5}$ & $1 / 18432$ & $1^{4} 2^{2} 3$ & $1 / 1152$ & $6^{2} 12$ & $1 / 288$ & $1^{2} 2^{2} 5$ & $1 / 80$ & 1214 & $1 / 28$ \\
\hline $1^{5} 2^{3}$ & $1 / 18432$ & $1^{2} 2^{4} 6$ & $1 / 1152$ & $12^{2}$ & $1 / 288$ & 12346 & $1 / 72$ & $1^{2} 7$ & $1 / 28$ \\
\hline $1^{4} 4^{2}$ & $1 / 18432$ & $1^{2} 36^{2}$ & $1 / 864$ & $1^{2} 2^{2} 3^{2}$ & $1 / 288$ & $4^{2} 12$ & $1 / 72$ & $2^{2} 14$ & $1 / 28$ \\
\hline $2^{4} 4^{2}$ & $1 / 18432$ & $2^{2} 3^{2} 6$ & $1 / 864$ & $3^{2} 12$ & $1 / 288$ & $1^{2} 2^{2} 8$ & $1 / 64$ & 12412 & $1 / 24$ \\
\hline $12^{5} 4$ & $1 / 15360$ & $124^{3}$ & $1 / 768$ & $1^{3} 2^{3} 4$ & $19 / 4608$ & 1235 & $1 / 60$ & 24 & $1 / 24$ \\
\hline $1^{5} 24$ & $1 / 15360$ & $1^{4} 2^{2} 4$ & $1 / 768$ & $12^{3} 46$ & $1 / 192$ & 12610 & $1 / 60$ & 20 & $1 / 20$ \\
\hline $12^{5} 6$ & $1 / 8640$ & $1^{2} 2^{4} 4$ & $1 / 768$ & $1^{3} 234$ & $1 / 192$ & $1^{2} 35$ & $1 / 60$ & 1248 & $5 / 64$ \\
\hline $1^{5} 23$ & $1 / 8640$ & $10^{2}$ & $1 / 600$ & $8^{2}$ & $1 / 192$ & $2^{2} 610$ & $1 / 60$ & & \\
\hline $2^{2} 6^{3}$ & $1 / 7776$ & $5^{2}$ & $1 / 600$ & $12^{3} 36$ & $1 / 144$ & 618 & $1 / 54$ & & \\
\hline $1^{2} 3^{3}$ & $1 / 7776$ & $12^{3} 34$ & $1 / 576$ & $1^{3} 236$ & $1 / 144$ & 39 & $1 / 54$ & & \\
\hline $1^{4} 2^{2} 6$ & $1 / 6912$ & $1^{3} 2^{3} 3$ & $1 / 576$ & $1^{2} 312$ & $1 / 144$ & 1238 & $1 / 48$ & & \\
\hline
\end{tabular}


Table C.5. The 160 nonzero $m=\mathrm{m}_{\mathrm{O}(\text { Leech })}(P)$ for $P$ in $\operatorname{Car}_{24}$.

\begin{tabular}{|c|c|c|c|c|c|c|c|}
\hline$P$ & $m$ & $P$ & $m$ & $P$ & $m$ & $P$ & $m$ \\
\hline $1^{24}$ & $1 / 8315553613086720000$ & $1^{4} 2^{2} 3^{6} 6^{3}$ & $1 / 31104$ & $24^{3}$ & $1 / 864$ & $1^{2} 2^{2} 6918^{2}$ & $1 / 108$ \\
\hline $2^{24}$ & $1 / 8315553613086720000$ & $1^{4} 5^{5}$ & $1 / 30000$ & $1^{4} 2^{4} 4^{4} 8^{2}$ & $1 / 768$ & $1^{2} 369^{2} 18$ & $1 / 108$ \\
\hline $3^{12}$ & $1 / 2690072985600$ & $2^{4} 10^{5}$ & $1 / 30000$ & $12^{2} 24^{2}$ & $1 / 576$ & $2^{2} 36918^{2}$ & $1 / 108$ \\
\hline $6^{12}$ & $1 / 2690072985600$ & $5^{2} 10^{4}$ & $1 / 19200$ & $1^{4} 2^{4} 3^{4} 6^{4}$ & $1 / 576$ & $3^{2} 6^{2} 12^{2} 24$ & $1 / 96$ \\
\hline $1^{8} 2^{16}$ & $1 / 178362777600$ & $5^{4} 10^{2}$ & $1 / 19200$ & $1^{2} 2^{4} 3^{2} 6^{3} 12^{2}$ & $1 / 576$ & 84 & $1 / 84$ \\
\hline $1^{16} 2^{8}$ & $1 / 178362777600$ & $1^{6} 2^{6} 4^{6}$ & $1 / 15360$ & $1^{2} 34^{2} 6^{2} 12^{3}$ & $1 / 576$ & $8^{2} 24^{2}$ & $1 / 72$ \\
\hline $4^{12}$ & $1 / 2012774400$ & $1^{4} 4^{2} 8^{4}$ & $1 / 12288$ & $1^{4} 2^{2} 3^{3} 6^{2} 12^{2}$ & $1 / 576$ & 391236 & $1 / 72$ \\
\hline $1^{8} 4^{8}$ & $1 / 743178240$ & $2^{4} 4^{2} 8^{4}$ & $1 / 12288$ & $2^{2} 3^{2} 4^{2} 612^{3}$ & $1 / 576$ & 6121836 & $1 / 72$ \\
\hline $2^{8} 4^{8}$ & $1 / 743178240$ & $15^{3}$ & $1 / 10800$ & $1^{2} 2^{2} 34^{4} 612^{2}$ & $1 / 576$ & 35 & $1 / 70$ \\
\hline $1^{12} 2^{12}$ & $1 / 389283840$ & $30^{3}$ & $1 / 10800$ & $1^{4} 2^{4} 34^{2} 6^{3} 12$ & $1 / 576$ & 70 & $1 / 70$ \\
\hline $1^{12} 3^{6}$ & $1 / 117573120$ & $3^{2} 6^{2} 12^{4}$ & $1 / 9216$ & $1^{4} 2^{4} 3^{3} 4^{2} 612$ & $1 / 576$ & $3^{2} 33$ & $1 / 66$ \\
\hline $2^{12} 6^{6}$ & $1 / 117573120$ & $1^{4} 2^{4} 8^{4}$ & $1 / 6144$ & $21^{2}$ & $1 / 504$ & $6^{2} 66$ & $1 / 66$ \\
\hline $1^{6} 3^{9}$ & $1 / 25194240$ & $1^{2} 3^{3} 4^{2} 12^{3}$ & $1 / 5184$ & $42^{2}$ & $1 / 504$ & $1^{2} 2^{2} 816^{2}$ & $1 / 64$ \\
\hline $2^{6} 6^{9}$ & $1 / 25194240$ & $2^{2} 4^{2} 6^{3} 12^{3}$ & $1 / 5184$ & $36^{2} 918^{2}$ & $1 / 432$ & $12^{2} 60$ & $1 / 60$ \\
\hline $3^{4} 6^{8}$ & $1 / 19906560$ & $3^{4} 6^{4} 12^{2}$ & $1 / 4608$ & $3^{2} 69^{2} 18$ & $1 / 432$ & 2060 & $1 / 60$ \\
\hline $3^{8} 6^{4}$ & $1 / 19906560$ & $3^{3} 9^{3}$ & $1 / 3888$ & $3^{2} 1224^{2}$ & $1 / 384$ & $1^{2} 3515^{2}$ & $1 / 60$ \\
\hline $5^{6}$ & $1 / 6048000$ & $6^{3} 18^{3}$ & $1 / 3888$ & $6^{2} 1224^{2}$ & $1 / 384$ & $2^{2} 61030^{2}$ & $1 / 60$ \\
\hline $10^{6}$ & $1 / 6048000$ & $1^{4} 3^{2} 4^{4} 12^{2}$ & $1 / 3456$ & $5^{2} 15^{2}$ & $1 / 360$ & $1^{2} 351530$ & $1 / 60$ \\
\hline $1^{6} 2^{10} 4^{4}$ & $1 / 1474560$ & $1^{6} 2^{6} 3^{3} 6^{3}$ & $1 / 3456$ & $10^{2} 30^{2}$ & $1 / 360$ & $2^{2} 6101530$ & $1 / 60$ \\
\hline $1^{10} 2^{6} 4^{4}$ & $1 / 1474560$ & $2^{4} 4^{4} 6^{2} 12^{2}$ & $1 / 3456$ & $13^{2}$ & $1 / 312$ & $1^{2} 2^{2} 3561015$ & $1 / 60$ \\
\hline $1^{4} 2^{4} 4^{8}$ & $1 / 1179648$ & $1^{6} 7^{3}$ & $1 / 2352$ & $26^{2}$ & $1 / 312$ & $1^{2} 2^{2} 3561030$ & $1 / 60$ \\
\hline $1^{8} 3^{8}$ & $1 / 1088640$ & $2^{6} 14^{3}$ & $1 / 2352$ & $1^{4} 2^{4} 3^{2} 4^{2} 6^{2} 12$ & $1 / 288$ & $1^{2} 4^{2} 728$ & $1 / 56$ \\
\hline $2^{8} 6^{8}$ & $1 / 1088640$ & $1^{2} 2^{2} 4^{2} 8^{4}$ & $1 / 2048$ & $1530^{2}$ & $1 / 240$ & $2^{2} 4^{2} 1428$ & $1 / 56$ \\
\hline $12^{6}$ & $1 / 483840$ & $3^{4} 15^{2}$ & $1 / 1800$ & $15^{2} 30$ & $1 / 240$ & 52 & $1 / 52$ \\
\hline $1^{6} 3^{5} 6^{4}$ & $1 / 311040$ & $6^{4} 30^{2}$ & $1 / 1800$ & $1^{4} 3^{2} 5^{2} 15$ & $1 / 180$ & $1^{2} 2^{2} 368^{2} 24$ & $1 / 48$ \\
\hline $2^{6} 3^{4} 6^{5}$ & $1 / 311040$ & $1^{4} 2^{6} 4^{3} 8^{2}$ & $1 / 1536$ & $2^{4} 6^{2} 10^{2} 30$ & $1 / 180$ & $1^{2} 2^{2} 3^{2} 4^{2} 6^{2} 12^{2}$ & $1 / 48$ \\
\hline $1^{6} 2^{6} 36^{5}$ & $1 / 311040$ & $1^{6} 2^{4} 4^{3} 8^{2}$ & $1 / 1536$ & $28^{2}$ & $1 / 168$ & $1^{2} 2^{2} 346^{2} 1224$ & $1 / 48$ \\
\hline $1^{6} 2^{6} 3^{5} 6$ & $1 / 311040$ & $4^{4} 12^{4}$ & $1 / 1440$ & $1^{4} 2^{4} 5^{2} 10^{2}$ & $1 / 160$ & $1^{2} 2^{2} 3^{2} 461224$ & $1 / 48$ \\
\hline $1^{8} 2^{8} 4^{4}$ & $1 / 294912$ & $20^{3}$ & $1 / 1200$ & $4^{2} 8^{2} 1224$ & $1 / 144$ & $1^{2} 3^{2} 721$ & $1 / 42$ \\
\hline $3^{4} 12^{4}$ & $1 / 276480$ & $4^{4} 20^{2}$ & $1 / 1200$ & $1^{4} 11^{2}$ & $1 / 132$ & $2^{2} 6^{2} 1442$ & $1 / 42$ \\
\hline $6^{4} 12^{4}$ & $1 / 276480$ & $1^{4} 5^{3} 10^{2}$ & $1 / 1200$ & $2^{4} 22^{2}$ & $1 / 132$ & 2040 & $1 / 40$ \\
\hline $4^{4} 8^{4}$ & $1 / 92160$ & $2^{4} 5^{2} 10^{3}$ & $1 / 1200$ & $1^{2} 4816^{2}$ & $1 / 128$ & $1^{2} 2^{2} 510^{2} 20$ & $1 / 40$ \\
\hline $1^{8} 5^{4}$ & $1 / 72000$ & $1^{4} 2^{4} 510^{3}$ & $1 / 1200$ & $2^{2} 4816^{2}$ & $1 / 128$ & $1^{2} 2^{2} 5^{2} 1020$ & $1 / 40$ \\
\hline $2^{8} 10^{4}$ & $1 / 72000$ & $1^{4} 2^{4} 5^{3} 10$ & $1 / 1200$ & $7^{2} 21$ & $1 / 126$ & $1^{2} 2^{2} 4^{2} 51020$ & $1 / 40$ \\
\hline $8^{6}$ & $1 / 48384$ & $1^{2} 3^{2} 9^{3}$ & $1 / 972$ & $14^{2} 42$ & $1 / 126$ & 39 & $1 / 39$ \\
\hline $1^{4} 2^{8} 3^{2} 6^{4}$ & $1 / 41472$ & $1^{4} 39^{3}$ & $1 / 972$ & 1560 & $1 / 120$ & 78 & $1 / 39$ \\
\hline $1^{8} 2^{4} 3^{4} 6^{2}$ & $1 / 41472$ & $2^{2} 6^{2} 18^{3}$ & $1 / 972$ & 3060 & $1 / 120$ & 56 & $1 / 28$ \\
\hline $7^{4}$ & $1 / 35280$ & $2^{4} 618^{3}$ & $1 / 972$ & $1^{2} 2^{4} 714^{2}$ & $1 / 112$ & $1^{2} 23$ & $1 / 23$ \\
\hline $14^{4}$ & $1 / 35280$ & $5^{2} 20^{2}$ & $1 / 960$ & $1^{4} 2^{2} 7^{2} 14$ & $1 / 112$ & $2^{2} 46$ & $1 / 23$ \\
\hline $1^{2} 2^{4} 3^{3} 6^{6}$ & $1 / 31104$ & $10^{2} 20^{2}$ & $1 / 960$ & $1^{2} 2^{2} 39^{2} 18$ & $1 / 108$ & $1^{2} 2^{2} 1122$ & $1 / 22$ \\
\hline
\end{tabular}


TABLE C.6. The nonzero dimensions of $\mathrm{M}_{\mathrm{W}_{\lambda}}\left(\mathrm{O}_{24}\right)$ for $\lambda_{1} \leq 3$.

\begin{tabular}{|c|c|c|c|c|c|c|c|c|c|}
\hline$\lambda$ & $\operatorname{dim}$ & $\lambda$ & $\operatorname{dim}$ & $\lambda$ & $\operatorname{dim}$ & $\lambda$ & $\operatorname{dim}$ & $\lambda$ & $\operatorname{dim}$ \\
\hline & $24: 1$ & $3^{2}$ & $4: 0$ & $3^{3} 2^{4} 1$ & $181: 0$ & $3^{4} 2^{8}$ & 148 & $3^{6} 2^{6}$ & 276 \\
\hline $1^{8}$ & $1: 1$ & $3^{2} 1^{2}$ & $19: 0$ & $3^{3} 2^{4} 1^{3}$ & $97: 0$ & $3^{5} 1$ & $27: 0$ & $3^{7} 1$ & $174: 0$ \\
\hline $1^{12}$ & 1 & $3^{2} 2$ & $3: 0$ & $3^{3} 2^{4} 1^{5}$ & 1 & $3^{5} 1^{3}$ & $94: 1$ & $3^{7} 1^{3}$ & $333: 17$ \\
\hline 2 & $9: 0$ & $3^{2} 21^{2}$ & $19: 0$ & $3^{3} 2^{5} 1$ & $251: 1$ & $3^{5} 1^{5}$ & $20: 0$ & $3^{7} 1^{5}$ & 211 \\
\hline $2^{2}$ & $27: 0$ & $3^{2} 2^{2}$ & $15: 0$ & $3^{3} 2^{5} 1^{3}$ & $120: 1$ & $3^{5} 1^{7}$ & 1 & $3^{7} 21$ & $512: 17$ \\
\hline $2^{3}$ & $26: 0$ & $3^{2} 2^{2} 1^{2}$ & $50: 0$ & $3^{3} 2^{6} 1$ & $265: 1$ & $3^{5} 21$ & $140: 0$ & $3^{7} 21^{3}$ & $801: 342$ \\
\hline $2^{4}$ & $43: 0$ & $3^{2} 2^{3}$ & $18: 0$ & $3^{3} 2^{6} 1^{3}$ & 100 & $3^{5} 21^{3}$ & $242: 1$ & $3^{7} 2^{2} 1$ & $905: 253$ \\
\hline $2^{4} 1^{8}$ & 1 & $3^{2} 2^{3} 1^{2}$ & $58: 0$ & $3^{3} 2^{7} 1$ & $219: 51$ & $3^{5} 21^{5}$ & $82: 1$ & $3^{7} 2^{2} 1^{3}$ & 927 \\
\hline $2^{5}$ & $35: 0$ & $3^{2} 2^{4}$ & $46: 0$ & $3^{3} 2^{8} 1$ & 134 & $3^{5} 2^{2} 1$ & $308: 0$ & $3^{7} 2^{3} 1$ & $1042: 683$ \\
\hline $2^{5} 1^{4}$ & $1: 1$ & $3^{2} 2^{4} 1^{2}$ & $97: 0$ & $3^{4}$ & $28: 0$ & $3^{5} 2^{2} 1^{3}$ & $417: 1$ & $3^{7} 2^{4} 1$ & 675 \\
\hline $2^{6}$ & $67: 1$ & $3^{2} 2^{4} 1^{6}$ & 1 & $3^{4} 1^{2}$ & $28: 0$ & $3^{5} 2^{2} 1^{5}$ & 87 & $3^{8}$ & $191: 34$ \\
\hline $2^{6} 1^{4}$ & $1: 1$ & $3^{2} 2^{5}$ & $48: 0$ & $3^{4} 1^{4}$ & $53: 1$ & $3^{5} 2^{3} 1$ & $546: 1$ & $3^{8} 1^{2}$ & $476: 137$ \\
\hline $2^{6} 1^{6}$ & 1 & $3^{2} 2^{5} 1^{2}$ & $91: 0$ & $3^{4} 1^{8}$ & 1 & $3^{5} 2^{3} 1^{3}$ & $551: 111$ & $3^{8} 1^{4}$ & 530 \\
\hline $2^{7}$ & $42: 0$ & $3^{2} 2^{6}$ & $97: 0$ & $3^{4} 2$ & $30: 0$ & $3^{5} 2^{4} 1$ & $672: 58$ & $3^{8} 2$ & $327: 51$ \\
\hline $2^{8}$ & $69: 1$ & $3^{2} 2^{6} 1^{2}$ & $123: 1$ & $3^{4} 21^{2}$ & $80: 0$ & $3^{5} 2^{4} 1^{3}$ & 525 & $3^{8} 21^{2}$ & $881: 552$ \\
\hline $2^{8} 1^{4}$ & 1 & $3^{2} 2^{6} 1^{4}$ & 1 & $3^{4} 21^{4}$ & $51: 0$ & $3^{5} 2^{5} 1$ & $659: 325$ & $3^{8} 2^{2}$ & $660: 333$ \\
\hline $2^{9}$ & $37: 0$ & $3^{2} 2^{7}$ & $70: 0$ & $3^{4} 21^{6}$ & $1: 1$ & $3^{5} 2^{6} 1$ & 398 & $3^{8} 2^{2} 1^{2}$ & 1047 \\
\hline $2^{10}$ & $48: 0$ & $3^{2} 2^{7} 1^{2}$ & $74: 0$ & $3^{4} 2^{2}$ & $112: 1$ & $3^{6}$ & $36: 0$ & $3^{8} 2^{3}$ & $500: 364$ \\
\hline $2^{11}$ & $11: 0$ & $3^{2} 2^{8}$ & $104: 0$ & $3^{4} 2^{2} 1^{2}$ & $202: 1$ & $3^{6} 1^{2}$ & $217: 1$ & $3^{8} 2^{4}$ & 346 \\
\hline $2^{12}$ & 37 & $3^{2} 2^{8} 1^{2}$ & 86 & $3^{4} 2^{2} 1^{4}$ & $132: 2$ & $3^{6} 1^{4}$ & $180: 0$ & $3^{9} 1$ & $307: 133$ \\
\hline 31 & $1: 0$ & $3^{2} 2^{9}$ & $39: 8$ & $3^{4} 2^{2} 1^{6}$ & 1 & $3^{6} 1^{6}$ & 91 & $3^{9} 1^{3}$ & 496 \\
\hline 321 & $7: 0$ & $3^{2} 2^{10}$ & 54 & $3^{4} 2^{3}$ & $155: 0$ & $3^{6} 2$ & $79: 0$ & $3^{9} 21$ & $651: 491$ \\
\hline $32^{2} 1$ & $11: 0$ & $3^{3} 1$ & $8: 0$ & $3^{4} 2^{3} 1^{2}$ & $291: 0$ & $3^{6} 21^{2}$ & $474: 0$ & $3^{9} 2^{2} 1$ & 542 \\
\hline $32^{3} 1$ & $31: 0$ & $3^{3} 1^{3}$ & $6: 0$ & $3^{4} 2^{3} 1^{4}$ & $126: 1$ & $3^{6} 21^{4}$ & $367: 61$ & $3^{10}$ & $158: 121$ \\
\hline $32^{4} 1$ & $33: 0$ & $3^{3} 21$ & $25: 0$ & $3^{4} 2^{4}$ & $293: 1$ & $3^{6} 2^{2}$ & $270: 0$ & $3^{10} 1^{2}$ & 406 \\
\hline $32^{5} 1$ & $56: 0$ & $3^{3} 21^{3}$ & $33: 0$ & $3^{4} 2^{4} 1^{2}$ & $432: 1$ & $3^{6} 2^{2} 1^{2}$ & $902: 93$ & $3^{10} 2$ & $177: 160$ \\
\hline $32^{6} 1$ & $61: 0$ & $3^{3} 2^{2} 1$ & $67: 0$ & $3^{4} 2^{4} 1^{4}$ & 156 & $3^{6} 2^{2} 1^{4}$ & 551 & $3^{10} 2^{2}$ & 161 \\
\hline $32^{7} 1$ & $63: 0$ & $3^{3} 2^{2} 1^{3}$ & $49: 0$ & $3^{4} 2^{5}$ & $270: 0$ & $3^{6} 2^{3}$ & $386: 16$ & $3^{11} 1$ & 93 \\
\hline $32^{8} 1$ & $59: 0$ & $3^{3} 2^{2} 1^{5}$ & $1: 1$ & $3^{4} 2^{5} 1^{2}$ & $387: 75$ & $3^{6} 2^{3} 1^{2}$ & $988: 418$ & $3^{12}$ & 74 \\
\hline $32^{8} 1^{3}$ & 1 & $3^{3} 2^{3} 1$ & $122: 0$ & $3^{4} 2^{6}$ & $380: 73$ & $3^{6} 2^{4}$ & $563: 197$ & & \\
\hline $32^{9} 1$ & $53: 0$ & $3^{3} 2^{3} 1^{3}$ & $102: 1$ & $3^{4} 2^{6} 1^{2}$ & 362 & $3^{6} 2^{4} 1^{2}$ & 948 & & \\
\hline $32^{10} 1$ & 18 & $3^{3} 2^{3} 1^{5}$ & $1: 1$ & $3^{4} 2^{7}$ & $192: 89$ & $3^{6} 2^{5}$ & $371: 286$ & & \\
\hline
\end{tabular}


See the discussion after Theorem 1 for instructions to read Table C.6.

\begin{abstract}
Aknowledgements. We thank Jean Lannes and Olivier Taïbi for useful discussions, the LMO for sharing the machine pascaline, the referee for interesting remarks, as well as Bill Allombert for answering our questions on PARI/GP.
\end{abstract}

\title{
References
}

[1] E. BAYER-Fluckiger, "Definite unimodular lattices having an automorphism of given characteristic polynomial", Comment. Math. Helv. 59 (1984), p. 509-538.

[2] E. Bayer-Fluckiger \& L. TAelman, "Automorphisms of even unimodular lattices and equivariant Witt groups", https://arxiv.org/abs/1708.05540, to appear in J. Eur. Math. Soc., 2017.

[3] R. E. Borcherds, "Classification of positive definite lattices", Duke Math. J. 105 (2000), no. 3, p. 525-567.

[4] N. Bourbaki, Groupes et algèbres de Lie, Chapitres 4, 5 et 6, Éléments de mathématique, Masson, 1981.

[5] R. W. CARTer, "Conjugacy classes in the Weyl group", Compos. Math. 25 (1972), p. 1-59.

[6] G. Chenevier, "Characteristic masses of lattices", http://gaetan.chenevier.perso.math. cnrs.fr/charmasses.

[7] G. Chenevier \& L. Clozel, "Corps de nombres peu ramifiés et formes automorphes autoduales", J. Am. Math. Soc. 22 (2009), no. 2, p. 467-519.

[8] G. Chenevier \& J. Lannes, Automorphic forms and even unimodular lattices, Ergebnisse der Mathematik und ihrer Grenzgebiete. 3. Folge., vol. 69, Springer, 2019.

[9] G. Chenevier \& D. RenARD, Level one algebraic cusp forms of classical groups of small rank, Memoirs of the American Mathematical Society, vol. 1121, American Mathematical Society, 2015.

[10] G. Chenevier \& O. TAÏBI, "Siegel modular forms of weight 13 and the Leech lattice", https://arxiv.org/abs/1907.08781, 2019.

[11] — "Discrete series multiplicities for classical groups over Z and level 1 algebraic cusp forms", Publ. Math., Inst. Hautes Étud. Sci. 131 (2020), p. 261-323.

[12] M. Cheng, J. F. R. Duncan \& J. A. Harvey, "Umbral moonshine and the Niemeier lattices", Res. Math. Sci. 1 (2014), article ID 3 (81 pages).

[13] A. M. Cohen, "Finite complex reflection groups", Ann. Sci. Éc. Norm. Supér. 9 (1976), p. 379-436, erratum in ibid. 11 (1978), no. 4, p. 613.

[14] - "Finite quaternionic reflection groups", J. Algebra 64 (1980), p. 293-324.

[15] J. H. Conway, "A group of order 8,315,553,613,086,720,000", Bull. Lond. Math. Soc. 1 (1979), p. 79-88.

[16] J. H. Conway, R. T. Curtis, S. P. Norton, R. A. Parker \& R. A. Wilson, Atlas of finite groups. Maximal subgroups and ordinary characters for simple groups, Oxford University Press, 1985, with computational assistance from J. G. Thackray, xxxiv +252 pages.

[17] J. H. Conway \& N. J. A. Sloane, "Low-dimensional lattices. IV. The mass formula", Proc. R. Soc. Lond., Ser. A 419 (1988), no. 1857, p. 259-286.

[18] - Sphere packings, lattices and groups, Grundlehren der Mathematischen Wissenschaften, vol. 290, Springer, 1999.

[19] L. DembéLÉ, "On the computation of algebraic modular forms on compact inner forms of GSp 4 ", Math. Comput. 83 (2014), no. 288, p. 1931-1950.

[20] N. Dummigan, "A simple trace formula for algebraic modular forms", Exp. Math. 22 (2013), no. 2, p. 123-131.

[21] W. Ebeling, Lattices and Codes. A course partially based on lectures by F. Hirzebruch, Advanced Lectures in Mathematics, Vieweg, 2002.

[22] V. A. Erokhin, "Groups of automorphisms of 24-dimensional even unimodular lattices", Zap. Nauchn. Semin. Leningr. Otd. Mat. Inst. Steklova 116 (1982), p. 68-73. 
[23] U. Fincke \& M. Pohst, "Improved methods for calculating vectors of short length in a lattice, including a complexity analysis", Math. Comput. 44 (1985), p. 463-471.

[24] G. Frobenius, "Über die Charaktere der mehrfach transitiven Gruppen", Berl. Ber. 1904 (1904), p. 558-571.

[25] W. Fulton \& J. Harris, Representation theory. A first course, Graduate Texts in Mathematics, vol. 129, Springer, 1991.

[26] The GAP Group, "GAP — Groups, Algorithms, and Programming, Version 4.10.2", 2019, http://www .gap-system.org.

[27] M. Greenberg \& J. Voight, "Lattice methods for algebraic modular forms on classical groups", in Computations with modular forms, Contributions in Mathematical and Computational Sciences, vol. 6, Springer, 2014, p. 147-179.

[28] B. Gross \& C. MCMullen, "Automorphisms of even unimodular lattices and unramified Salem numbers", J. Algebra 257 (2002), no. 2, p. 265-290.

[29] M. Hall, "Note on the Mathieu group M12", Arch. Math. 13 (1962), p. 334-240.

[30] A. Hulpke, "Conjugacy classes algorithms in finite permutation groups via homomorphic images", Math. Comput. 69 (2000), no. 232, p. 1633-1651.

[31] M. H. Ingraham, "A note on determinants", Bull. Am. Math. Soc. 43 (1937), p. 579-580.

[32] M. Kneser, "Klassenzahlen definiter quadratischer formen", Arch. Math. 8 (1957), p. 241250.

[33] K. KoIKe \& I. TeradA, "Young-diagrammatic methods for the representation theory of the classical groups of type $\mathrm{B}_{n}, \mathrm{C}_{n}, \mathrm{D}_{n}$ ", J. Algebra 107 (1987), p. 466-511.

[34] B. Kostant, "Lie Algebra Cohomology and the Generalized Borel-Weil Theorem", Ann. Math. 74 (1961), p. 320-387.

[35] J. Lansky \& D. Pollack, "Hecke algebras and automorphic forms", Compos. Math. 130 (2002), no. 1, p. 21-48.

[36] D. Loeffler, "Explicit Calculations of Automorphic Forms for Definite Unitary Groups", LMS J. Comput. Math. 11 (2010), p. 326-342.

[37] G. NeBE, "On automorphisms of extremal even unimodular lattices", Int. J. Number Theory 9 (2013), no. 8, p. 1933-1959.

[38] H.-V. Niemeier, "Definite quadratische Formen der Dimension 24 und Diskriminante 1", J. Number Theory 5 (1973), p. 142-178.

[39] The PARI Group, "PARI/GP version 2.11.0", 2014, available from http://pari.math. u-bordeaux.fr/.

[40] W. Plesken \& B. Souvignier, "Computing isometries of lattices", J. Symb. Comput. (1997), p. 327-334.

[41] J. Schur, "Über die Darstellung der symmetrischen und der alternierenden Gruppe durch gebrochene linear Substitutionen", J. für Math. 139 (1911), p. 155-250.

[42] N. J. A. SlOANE, "The On-Line Encyclopedia of Integer Sequences", 2010, http://oeis .org.

[43] O. TAїBI, "Dimensions of spaces of level one automorphic forms for split classical groups using the trace formula", Ann. Sci. Éc. Norm. Supér. 50 (2017), no. 2, p. 269-344.

[44] B. B. VENKOv, "On the classification of integral even unimodular 24-dimensional quadratic forms", 1999, Chapter 18 in [18].

[45] R. WendT, "Weyl's character formula for non-connected Lie groups and orbital theory for twisted affine Lie algebras", J. Funct. Anal. 180 (2001), no. 1, p. 31-65.

[46] H. Weyl, The Classical Groups, Princeton Mathematical Series, vol. 1, Princeton University Press, 1946.

Gaëtan Chenevier

CNRS, Université Paris-Saclay

Laboratoire de mathématiques d'Orsay

91405 Orsay, France

E-mail: gaetan.chenevier@math.cnrs.fr

$U R L:$ http://gaetan. chenevier.perso.math.cnrs.fr/ 